\title{
Generation and Characterization of Anisotropic Microstructures in Rare Earth-Iron-Boron Alloys
}

\author{
by \\ Nathaniel Oster \\ A dissertation submitted to the graduate faculty \\ in partial fulfillment of the requirements for the degree of \\ DOCTOR OF PHILOSOPHY
}

\author{
Major: Materials Science \& Engineering \\ Program of Study Committee: \\ Iver Anderson, Major Professor \\ R.W. McCallum \\ Matthew Kramer \\ Ralph Napolitano \\ Frank Peters
}

lowa State University

Ames, IA

2012

Copyright (@ Nathaniel Thorland Oster, 2012. All rights reserved 


\section{Table of Contents}

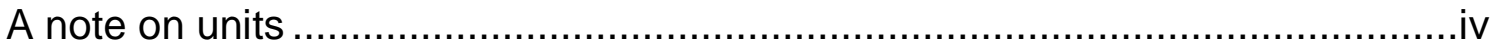

Chapter 1 - Problem statement and approach.......................................... 1

1.1 - The need for high temperature, anisotropic magnet material........ 1

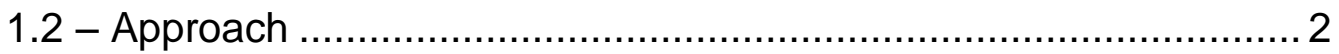

Chapter 2 - Background information......................................................... 4

2.1 - What is a permanent magnet? ............................................. 4

2.2 - Magnetic Domain Energetics .............................................. 6

2.3 - Intrinsic vs. extrinsic properties in magnetic materials ............... 12

Chapter 3 - Rare earth-iron-boron structure, properties, and processing ......... 14

$3.1-\mathrm{Nd}_{2} \mathrm{Fe}_{14} \mathrm{~B}$ discovery, structure, and intrinsic properties.............. 14

3.2 - Coercivity mechanisms ........................................................... 16

3.3 - Isotropic vs. anisotropic magnets............................................. 18

3.4 - Processing sintered magnets.................................................... 21

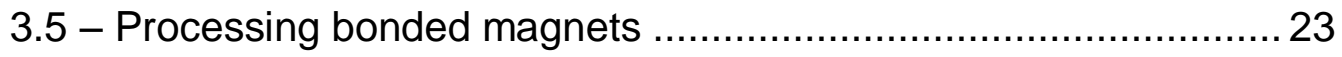

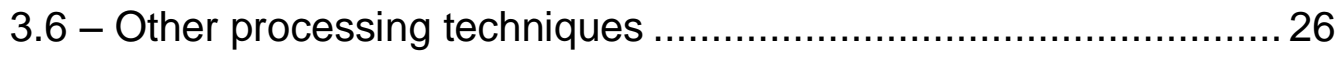

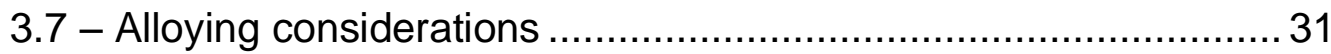

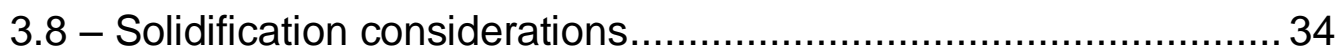

Chapter 4 - The effects of applied uniaxial stress during devitrification on

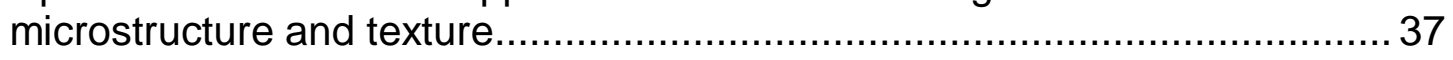

4.1 - Experimental Methods ....................................................... 38

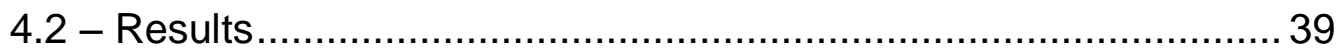

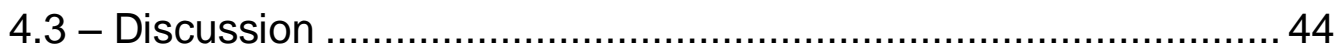

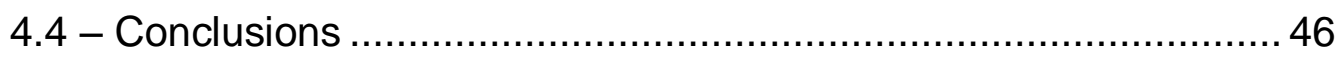

Chapter 5 - Effects of wheel surface conditions on phase assemblage and glass formation in melt-spun TiC-modified $\mathrm{Nd}_{2} \mathrm{Fe}_{14} \mathrm{~B}$................................ 47

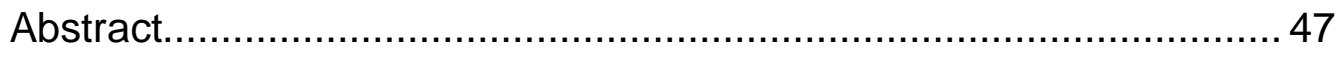

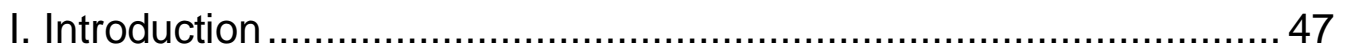

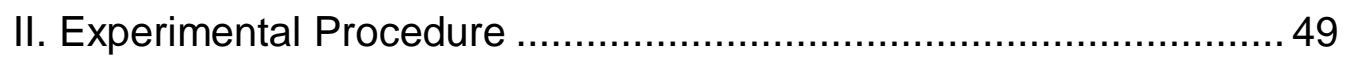

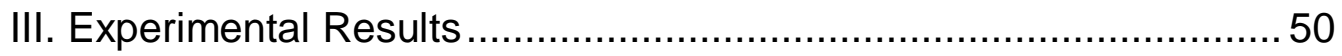

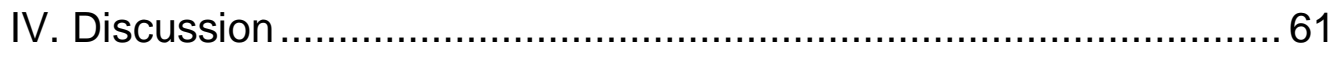

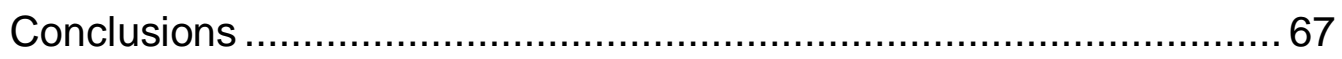

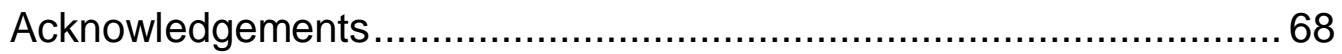


Chapter 6 - Effects of Ag additions on melt-spun $\mathrm{RE}_{2} \mathrm{Fe}_{14} \mathrm{~B}$ microstructure and texture 70

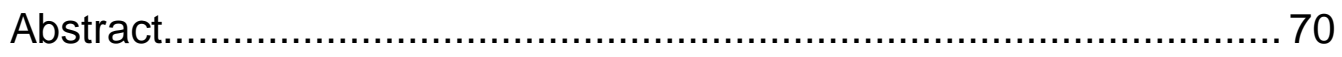

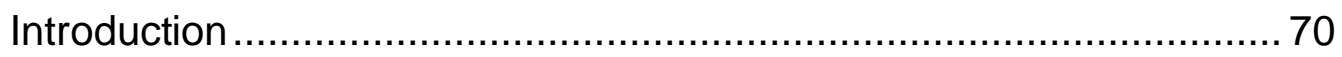

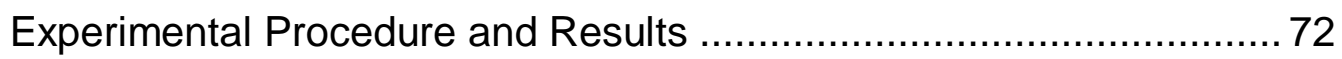

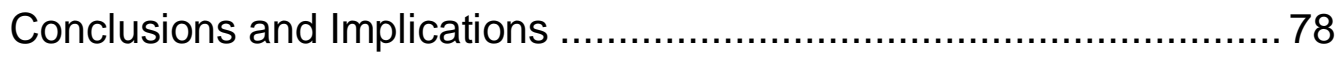

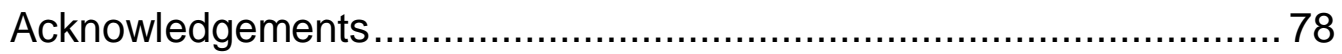

Chapter 7 - Alteration of the solidification microstructure and texture of melt-spun RE-Fe-B alloys by the addition of Ag ..................................... 79

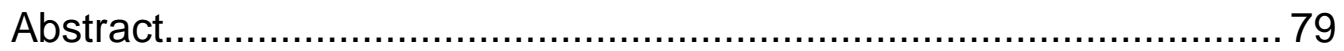

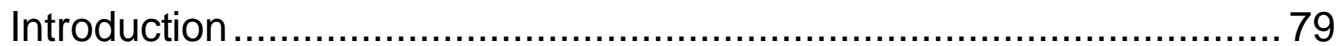

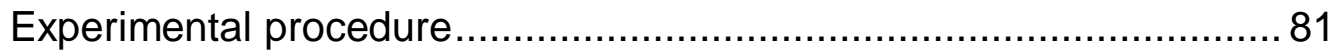

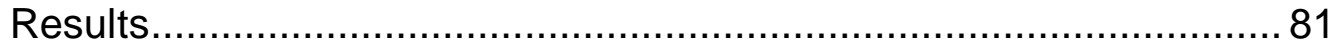

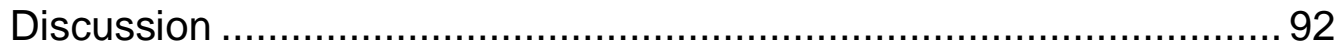

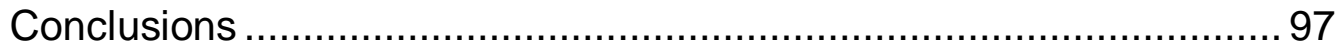

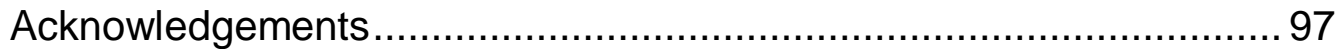

Chapter 8 - General Conclusions and Future Directions .................................. 98

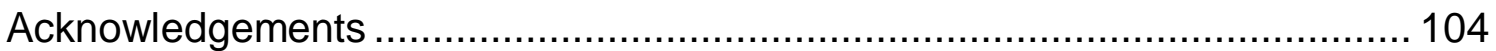

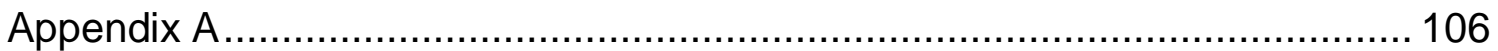

A.1 - Initial studies on other additives ............................................ 106

A. 2 - Orientation imaging microscopy of Nd-Y-Fe-B-Ag alloys.......... 109

Appendix $B-A$ simple model for wheel profile and its effects on heat flow

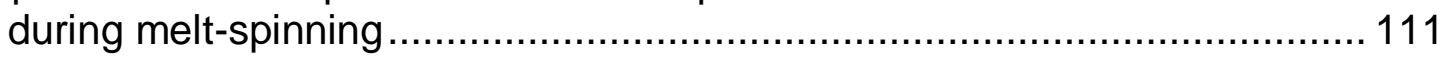

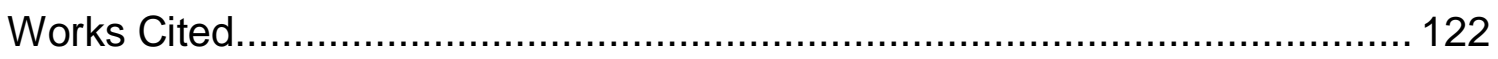




\section{A note on units}

The proper units for discussing magnetism are often disputed within the magnetic community. Classically, cgs (centimeter-gram-second) units were preferred over SI units. There is, however, a growing use of SI units. While both unit systems are equally valid, the formulation often changes between the unit systems. For example, the equation for magnetic flux density in cgs units is:

$$
B=\mu_{0}(H+4 \pi M)
$$

While magnetic flux density in SI units is:

$$
B=\mu_{0}(H+M) \text {. }
$$

Due to this confusion, a single unit system should be chosen. In this document, formulation will be presented in cgs units unless otherwise noted. 


\section{Chapter 1 - Problem statement and approach}

\section{1 - The need for high temperature, anisotropic magnet material}

In recent years there has been an increasing demand for electrification of the automotive fleet, through the implementation of electric or hybrid vehicles. In order to realize this change, a transition from fossil fuel-powered combustion engines to electric drive motors is required. An electric motor for these applications can be achieved in various ways, but a number of engineering challenges exist. The motor must be capable of operating at high temperatures and exhibit high torque, while minimizing cost and weight.

Within an electric motor, the magnetic field can be generated through either an electrically excited coil set or a permanent magnet (PM). Gieras and Wang $[1,2]$ identify the following benefits to using a PM motor: no excitation losses are present since only the stator coils need be electrically excited, higher torque per volume is produced, maintenance is reduced, and better dynamic performance is exhibited as a result of a higher magnetic flux density in the air gap. Due to these reasons, among others, brushless PM motors are preferred for use in automotive traction motors.

A number of additional constraints are present in automotive applications. The allotted space for the motors is limited, requiring high torque-to-volume ratios. This requires materials with high magnetic flux density that can resist demagnetization. As a result, neodymium-iron-boron materials are typically used. The magnetic flux density can also be greatly increased by using an anisotropic magnet instead of an isotropic one. In section 2.3 this will be described in further detail. Additionally, the motors can produce a great deal of heat. This can pose a problem for traditional $\mathrm{Nd}-\mathrm{Fe}-\mathrm{B}$ materials, which typically have an operating limit of $\sim 120^{\circ} \mathrm{C}$ [3]. By improving the temperature performance of the magnets, the use of cooling systems can be minimized, if not eliminated. Isotropic, high temperature performance alloys for use in traction motors have been created by Anderson, McCallum, and Kramer [4]. In a recent report by Dr. Peter Campbell [5], the following conclusion was made: 
"Ames Laboratory has developed an alloy with improved temperature stability for use in bonded $\mathrm{NdFeB}$ magnets, and if it were possible to moderate its dysprosium content while developing grain alignment to turn this into an anisotropic powder, it would be certainly be preferable to HDDR powder as a compression-molded magnet for the IPM motor."

A need still exists for improving the magnet material used in these high torque electric motors. By utilizing an alloy similar to the one developed by the Ames Laboratory group, it would be possible to further improve the material through careful control of the microstructure to obtain an anisotropic material suitable for use in bonded magnets for traction motor use. The goal of this work is to investigate methods in which anisotropy could be induced in fine-grained alloys similar to the one designed by Anderson, McCallum and Kramer.

\section{2 - Approach}

Materials suitable for use in RE-Fe-B anisotropic bonded magnets have several requirements in order to make them viable alternatives to their microcrystalline sintered magnet counterparts. The particulate, itself, must be able to maintain its magnetization even in the presence of demagnetizing fields. This means that the materials must have a grain size on the order of tens of nanometers to a few micrometers (further explanation of this is provided in chapter 3 ). In addition to the fine grain size, the particulate must display a strong texture, preferentially in the direction of easy magnetization. More information on the need for texture can be found in chapter 3 . Satisfying these requirements must be done through careful control of the microstructure.

We have identified two general processing routes to creating a fine, textured microstructure: form an amorphous precursor and devitrify in a manner that induces texture or form the fine, textured microstructure upon cooling directly from the liquid state. Since it is possible to form significant amounts of amorphous material in RE-Fe-B alloys, texture could be induced through biasing the orientation 
of the crystallites upon crystallization of the amorphous material. One method of creating this bias is to form glassy material and apply uniaxial pressure during crystallization. Experiments on this are presented in chapter 4.

All of the work presented here utilizes melt-spinning, either to create precursor material, or to achieve a desired final microstructure. To obtain greater control of the system to process these materials, a study was done on the effects of heating the wheel and modifying the wheel's surface finish on glass formation and phase selection (see chapter 5 ).

The second general approach—creating the desired microstructure directly from the liquid-can be done through directional rapid solidification. In particular, alloys melt-spun at low tangential wheel speeds often display directional columnar growth through a portion of the ribbon. By refining and stabilizing the columnar growth, a highly textured fine microstructure is achieved. The effects of adding a segregating element $(\mathrm{Ag})$ on the columnar growth are characterized and presented in Chapters 6 and 7. A flow chart describing the processing routes attempted in this work is shown below in Figure 1.

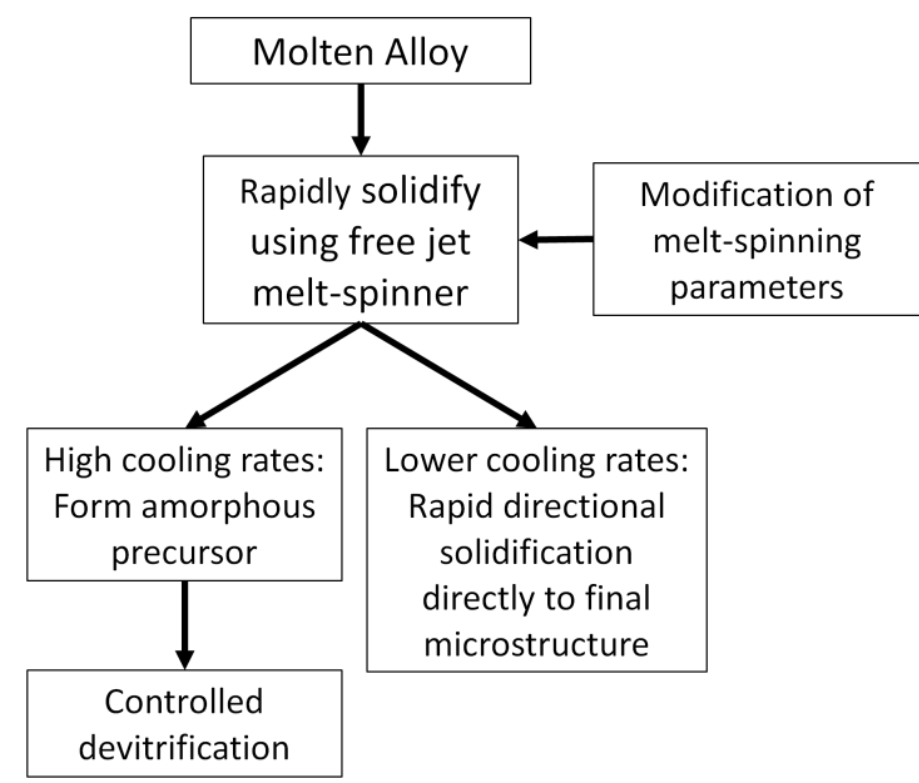

Figure 1 - Flow chart showing the proposed processing routes for achieving anisotropic RE-Fe-B material suitable for polymer-bonded magnets 


\section{Chapter 2 - Background information}

\section{1 - What is a permanent magnet?}

Permanent magnets are materials capable of maintaining their own persistent magnetic field in the absence of an applied field. They display magnetic hysteresis, the dependence of the system on prior exposure to magnetic fields. Figure 2 shows a hypothetical hysteresis loop for a permanent magnet. It is a measure of magnetization (M) vs. applied magnetic field $(\mathrm{H})$. This is effectively a measure of the internal response of the magnet to an applied field. It is particularly useful for characterizing the magnetic material itself. Hysteresis curves can also measure magnetic flux density $(B)$ vs. applied magnetic field $(H)$. This is an external measure of hysteresis, since it takes into account the presence of the external field. These curves are useful for characterizing the applications of magnetic materials, such as transformer cores or electric motors. $B$ and $M$ are related as follows [3]:

$$
B=\mu_{0}(H+4 \pi M)
$$

There are a number of parameters that are useful in characterizing a magnetic material, many of which are obtained from the hysteresis behavior. They include the following properties:

Saturation Magnetization $\left(\mathrm{M}_{\mathrm{S}}\right)$ : The maximum magnetization that a material can display once fully magnetized. On a hysteresis curve, it is denoted by the maximum magnetization value.

Remanent Magnetization $\left(M_{R}\right.$ or $\left.B_{R}\right)$ : The magnetization that persists once a material has been fully magnetized and the external field has been removed. This is denoted by the point where the hysteresis curve crosses the positive vertical axis.

Intrinsic Coercivity $\left(\mathrm{H}_{\mathrm{c} i}\right)$ : The opposing magnetic field required to completely demagnetize the material. It is denoted by the point where the $\mathrm{M}$ vs. $\mathrm{H}$ curve crosses the negative horizontal axis.

Technical Coercivity $\left(\mathrm{H}_{\mathrm{c}}\right)$ : The opposing magnetic field required to reduce the magnetic flux density to zero. It is denoted by the point where the $\mathrm{B}$ vs. $\mathrm{H}$ curve crosses the negative horizontal axis. 
Maximum Energy Product $\left(\mathrm{BH}_{\max }\right)$ : A measure of the maximum potential energy that can be stored within a magnet. This property is determined from the demagnetization behavior of the material. (See section 1.3 for more detail).

Curie Temperature $\left(T_{c}\right)$ : The temperature at which the material no longer displays spontaneous magnetic ordering and, therefore, hysteretic magnetic properties.

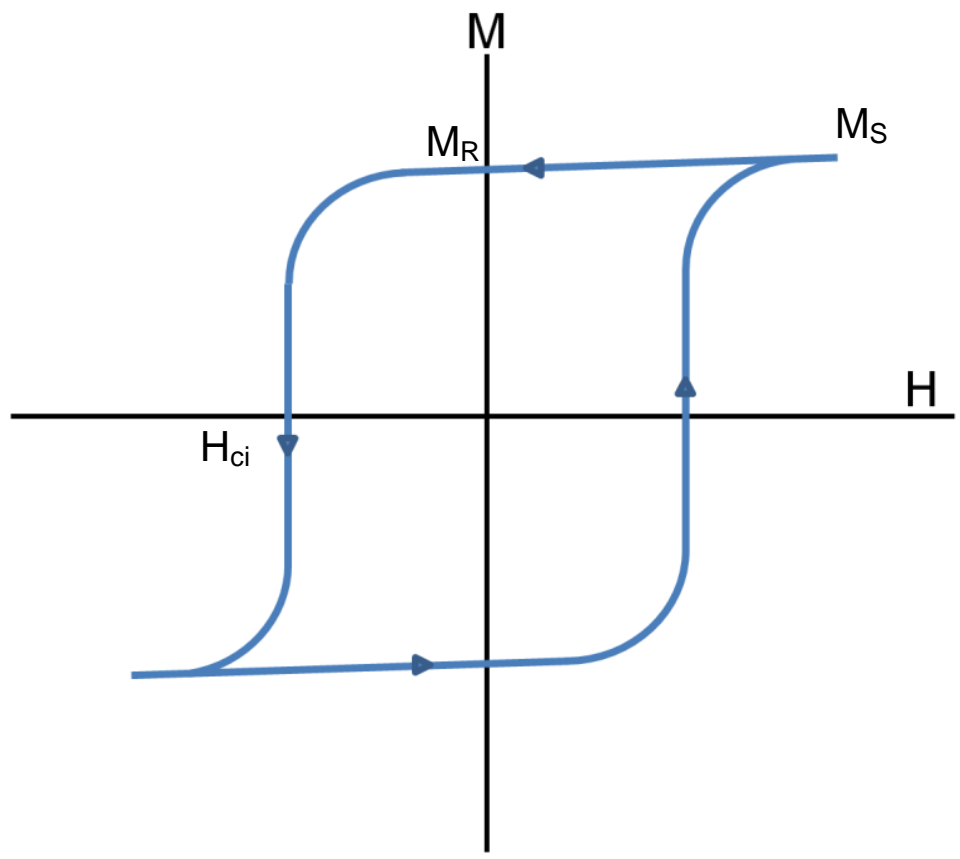

Figure 2 - Example Magnetization vs. Applied Field Hysteresis Curve

Of these properties, a few are of particular importance in the performance of a permanent magnet. A permanent magnet must be able to generate a strong external field when no applied field is present. Therefore remanent magnetization is of critical importance. For most applications, the permanent magnet will be exposed to a demagnetizing environment. To be of use, the material must be able to resist demagnetization, so coercivity is also greatly important. A Curie temperature significantly above the intended operating temperature is necessary since a material must be ferromagnetic at the desired temperature in order to be of any use. 
However, the most used parameter in describing overall quality of a magnet is the maximum energy product, as it determines the maximum capacity of the material to store potential energy.

The hysteretic behavior of magnetic materials is caused by features called magnetic domains, which are local areas in which the magnetic moments of atoms are aligned. Magnetic domains will be further discussed in section 2.3. Materials which exhibit domains are called ferromagnets. When a magnetic field is applied to a ferromagnet, there is a driving force for the domains to align with the magnetic field. Favorably oriented domains will grow at the expense of unfavorably oriented ones. This can occur by existing domains growing or by new domains nucleating and growing. Once all the magnetic domains are aligned, the material is maximally magnetized-leading to saturation. The degree to which the domains remain aligned once the external field is removed determines the remanent magnetization.

Coercivity is determined by the ability of domain walls to nucleate and move within the material. If the walls are difficult to move or nucleate, larger fields will be required to magnetize and demagnetize the material and it will have a higher coercivity. More information will be presented in section 3.2.

Temperature performance of the magnet can also be discussed in terms of magnetic domains. If temperature is increased, the atoms gain thermal energy. This thermal energy competes with the exchange energy between the magnetic moments, weakening the ordering between neighboring atoms. As a result, both remanent magnetization and coercivity decrease as temperature increases. Once the Curie temperature is reached, the atoms have enough thermal energy to break free of the magnetic ordering completely, resulting in the transformation of the material from a ferromagnetic state to a paramagnetic state and the disappearance of hysteretic behavior.

\section{2 - Magnetic Domain Energetics}

Ferromagnetic behavior arises from the presence and dynamics of magnetic domains. Thermodynamically, the presence of domains results in a 
reduction in the overall free energy of the system. Four primary energies contribute to the free energy associated with magnetic domains [6].

One contribution to the free energy is known as exchange energy. It describes the interaction between neighboring magnetic moments. It is due to this energy that neighboring moments will couple and ferromagnetism exists. Without it, there would be no driving force for spontaneous alignment of magnetic moments, and therefore, ferromagnetism would not exist. The energy can be described as:

$$
E_{e x}=-2 \sum_{i>j} J_{i j} S^{2} \cos \varphi_{i j}
$$

Where $E_{e x}$ is the exchange energy, $J_{i j}$ is the exchange integral, $S$ is the magnitude of spin, and $\varphi_{i j}$ is the angle between neighboring spins. The exchange integral, $\mathrm{J}$, is derived from quantum mechanical calculations based on electronic structure.

The second energy is known as anisotropy energy, or magnetocrystalline anisotropy. This energy arises from interactions of the magnetic moments with the crystal lattice. In a crystalline ferromagnetic material, magnetic moments will more readily align with certain crystallographic directions than others. The axes magnetic moments preferentially align with, and therefore reduce free energy, are known as "easy axes." The energy for a uniaxial material can be described by the series expansion below:

$$
E_{K}=\sum_{n} K_{n} \sin ^{2 n}(\theta)
$$

Where $E_{K}$ is the anisotropy energy, $n$ is the number of series expansions, $K$ is an anisotropy constant (a material constant independent of $\theta$ ), and $\theta$ is the angle between the easy axis and direction of magnetization. It should also be noted that the values for K' are highly dependent on temperature. For materials with multiple easy axes, the formulation becomes more complex, but can be determined.

The third contribution to free energy is the magnetoelastic energy. This energy relates the effects of mechanical stress on magnetic energy. When stress is present, the material can have a preferred magnetization orientation. This is related by the equation below: 


$$
E_{m e}=\frac{3}{2} \lambda_{s} \sigma \sin ^{2}(\theta)
$$

Where $E_{m e}$ is the energy contribution due to magnetoelastic effects, $\lambda_{s}$ is the saturation magnetostriction, $\sigma$ is the applied stress, and $\theta$ is the angle between the applied stress and the direction of magnetization. For isotropic materials, the saturation magnetostriction is a constant. For anisotropic materials, the magnetostriction is a function of multiple material constants and the angle of applied stress relative to the lattice constants. Further description can be found elsewhere [3].

The fourth contribution to free energy is magnetostatic energy. Magnetostatic energy is the energy that is contributed from the magnetization of the material. It is expressed by the equation below:

$$
E_{m s}=-\frac{1}{2} \boldsymbol{H}_{\text {tot }} \cdot \boldsymbol{B}
$$

Where $E_{m s}$ is the magnetostatic energy, $\mathbf{H}_{\text {tot }}$ is the total magnetic field vector, and $\mathbf{B}$ is the magnetic flux density vector in the material. At this point, a distinction should be made between applied field and total field. The applied field is the external field applied to a material. The total field is actually a summation of two fields, the applied field and the demagnetizing field. Within a magnetic material, the demagnetizing field always points in the opposite direction as the direction of magnetization. The demagnetizing field can be described by the following equation:

$$
H_{d}=4 \pi \mu_{0} N_{d} M
$$

Where $H_{d}$ is the demagnetizing field, $\mu_{0}$ is the permeability of free space, $N_{d}$ is the demagnetizing factor (a constant based on sample geometry), and $\mathrm{M}$ is the magnetization of the material.

The energies described above can be used to explain the creation of magnetic domains and domain walls (the boundaries between neighboring domains). When an external field is applied, the magnetostatic energy is decreased if the magnetic moments align with the applied field. However, when the field is removed, the demagnetizing field contributes a driving force to reduce the overall magnetization. The individual moments, however, do not simply change from being 
ordered to disordered, since the exchange energy couples neighboring moments. Rather, the moments form regions of order, known as magnetic domains. A ferromagnet that appears demagnetized on a macroscopic scale still has magnetic order locally, in the form of domains. The orientation of the individual domains, however, can be random or anti-parallel, depending on the microstructure and magnetocrystalline anisotropy of the material.

Between neighboring domains, there forms a transition zone, known as a domain wall. Since the exchange energy increases with increasing angle between neighboring spins, having a sharp transition will increase the free energy of the system. As a result the transition is gradual. In a bulk material, the domain walls formed are known as Bloch walls. In these walls, the moments are gradually rotated out of the plane of the moments. This idea was proposed by Felix Bloch in 1932 [7]. A schematic of the Bloch wall moment orientations is shown below in Figure 3. In thin films, to reduce magnetostatic energy, a Néel wall is formed instead. In this type of domain wall (proposed by Louis Neel in 1944 [8]), the moments are gradually rotated in the plane of the moments. A schematic of a Néel wall is shown in Figure 4.

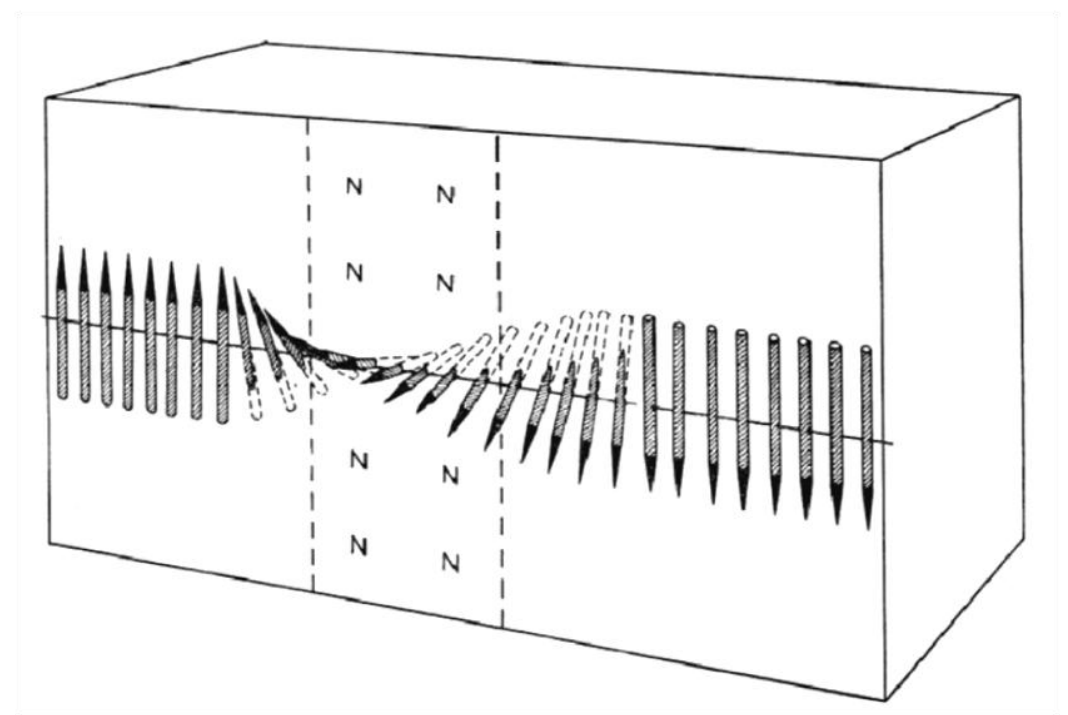

Figure 3 - Illustration of the magnetic moments in a Bloch wall [6]. 


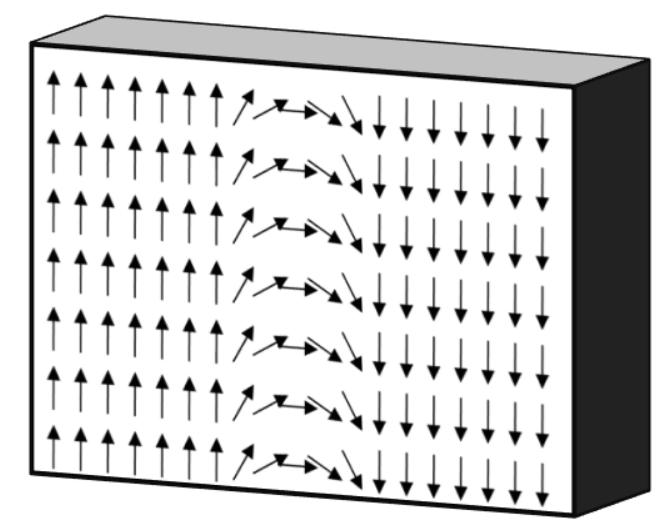

Figure 4 - Illustration of the magnetic moments in a Néel wall.

When a material is subjected to a demagnetizing field, there is a driving force to align the magnetic moments with the applied field in order to reduce the magnetostatic energy. One possibility, known as coherent rotation, is for the moments within an unfavorably oriented domain to simply rotate into the direction of the field. However, this does not occur easily in materials with any significant amount of magnetocrystalline anisotropy since it would require the bulk of the domain to transition through unfavorable orientations. Instead, domain walls will move as favorably oriented domains grow at the expense of their unfavorably oriented neighbors. Upon doing so, only a relatively small number of moments must be subjected to the high anisotropy energy. As more moments rotate into the unfavorable orientations, the previous occupants rotate to more favorable axes. As a result, the energy required to move a magnetic domain wall is less than that required to coherently rotate a whole domain.

Domains play a critical role in determining how a permanent magnet is processed. Since domain wall motion is the primary mechanism of changing the magnetization within a material, intrinsic coercivity is greatly affected by the ease of domain wall motion. The more difficult the domain walls are to move, the greater the observed intrinsic coercivity. An apt comparison can be made to strengthening of a metal, where strength is largely derived from limiting dislocation motion. In fact, the relationship even extends into the description of the two materials. Magnetic materials with high coercivity are known as "hard" magnets, while those with little 
coercivity are called "soft" magnets. This naming scheme was originally derived from the mechanical properties of early magnetic materials (i.e. steel and iron) [9]. The harder the steel was, the greater the observed coercivity. This occurs because impurities and strain in the lattice hinder domain wall movement, in addition to dislocation movement. Further detail on the processing used to limit domain wall motion is presented in Chapter 3.

Similar to nucleation kinetics in solidification, where bulk energy contributions compete with surface energy contributions to determine critical radius for nucleation, magnetic domain size is controlled by both bulk and surface energies. In this case, the magnetostatic energy can be thought of as the bulk energy contribution.

Likewise, energy is added to the system with the presence of domain walls. The domain wall energy is a summation of the exchange energy and the anisotropy energy of the crystal system over the volume of the domain wall. The domain wall energy, $\gamma$, can be written as follows:

$$
\gamma=2 \pi \sqrt{\frac{J S^{2} K}{a}}
$$

Where $\mathrm{y}$ is the domain wall energy per unit area, $\mathrm{J}$ is the exchange integral, $\mathrm{S}$ is the magnitude of spin, $\mathrm{K}$ is the anisotropy constant, and a is the lattice parameter. In particular, it is important to note that the domain wall energy increases with both increasing anisotropy and increasing exchange energy (which is related to $\mathrm{JS}^{2}$ ). By minimizing the sum of the two energies, the critical size of a magnetic domain of a given geometry can be calculated. If one assumes a spherical particle, it is energetically unfavorable for a domain wall to exist in a spherical particle with a radius smaller than $R_{c}$, where $R_{c}$ is calculated as follows:

$$
R_{c}=\frac{9 \gamma}{4 \pi M_{s}^{2}}
$$

As the particle is decreased, the presence of a domain wall becomes increasingly unfavorable. As a result, by decreasing particle size, coercivity can be enhanced. A model describing this phenomenon was developed by Kittel in 1948 [10]. Further description of this can be found in section 3.2. 


\section{3 - Intrinsic vs. extrinsic properties in magnetic materials}

The properties described in section 2.1 arise from a number of sources, both intrinsic and extrinsic. Some properties are derived from the composition and the crystal structure, itself, while some are based on the microstructure of the material in addition to the composition and crystal structure. Those properties that occur independently of microstructure are known as intrinsic. Intrinsic properties include magnetocrystalline anisotropy, saturation magnetization, and Curie temperature. If the property is dependent on microstructure or other external factors, it is extrinsic. Extrinsic properties include coercivity, remanent magnetization, and energy product. In magnetic materials, how a material is processed can greatly impact the extrinsic properties.

Intrinsic properties

Magnetocrystalline anisotropy is the degree to which magnetic domains have energetically favorable crystallographic directions in which to align. In high symmetry systems, such as Fe, there are a number of directions in which domains can easily align. For $\mathrm{Fe}$, the $<00 />$ family of directions are the "easy" axes, the directions which the material will spontaneously form domains. This means that in a single crystal of $\mathrm{Fe}$, domains can easily form in 6 different directions. In a system with lower symmetry, such as $\mathrm{Nd}_{2} \mathrm{Fe}_{14} \mathrm{~B}$, the easy axis occurs along the $<00 />$ directions in its tetragonal structure. This means that there are only 2 directions in which domains are energetically favored. By having fewer directions that can easily be magnetized, changing the domain structure requires greater energy.

Curie temperature is the temperature at which a ferromagnetic material transitions to a paramagnetic material. This is directly related to the exchange energy of the moments and is independent of microstructure.

\section{Extrinsic properties}

Coercivity is a function of both intrinsic factors (such as magnetocrystalline anisotropy) and extrinsic factors (i.e. microstructure). In a broader sense, it is the 
resistance to change of the magnetic domain structure. The coercivity is also greatly affected by microstructure. Two primary methods exist in which microstructure is manipulated to achieve higher coercivity. In one case, nucleation of new domains is minimized. In the other, domain walls are pinned. This will be further discussed in section 2.2.

Remanence is affected by saturation magnetization as well as by microstructure. The ideal permanent magnet would display the same magnetic flux density for the remanent magnetization as for the saturation magnetization. In this case, the magnetic domains would be perfectly aligned in the direction of magnetization. This would require all of the crystals in the bulk material to be perfectly textured. This is never achieved in practice. As the

Maximum energy product, or $\mathrm{BH}_{\max }$, is yet another extrinsic property. It is among the most commonly used figures of merit for permanent magnetic materials. The shape of the demagnetization curve $\left(2^{\text {nd }}\right.$ quadrant in a hysteresis loop) determines its value. The relationship between $B$ and $M$ (in CGS units) is as follows: $B=\mu_{0}(H+4 \pi M)$, where $B$ is the magnetic flux density, $\mu_{0}$ is the permeability of free space, $\mathrm{M}$ is magnetization, and $\mathrm{H}$ is applied magnetic field. Maximum energy product is the maximum value of $|\mathrm{BxH}|$ on the curve. In an ideal magnet, the hysteresis loop would be very square, with no loss of magnetization until the magnetization is reversed. If this is assumed, along with the magnet having ample coercivity, the theoretical maximum energy product can be calculated by $\mathrm{BH}_{\max }=(4$ $\left.\pi \mathrm{M}_{\mathrm{s}}\right)^{2} / 4$. However, these values are not observed in practice, primarily due to microstructure. This calculation merely gives an upper limit to the possible energy product.

While intrinsic properties give us clues as to the maximum possible performance a material could theoretically achieve, no commercial alloys are single crystal. As a result, processing and control of microstructure largely determine the properties observed in bulk magnetic materials. 


\section{Chapter 3 - Rare earth-iron-boron structure, properties, and processing}

Currently, the highest performance permanent magnets (at room temperature) available today are based on the $\mathrm{RE}_{2} \mathrm{Fe}_{14} \mathrm{~B}$ system. Within this system there are two routes typically used to obtain the desired properties, each using a different mechanism to maintain coercivity. In aligned and sintered magnets, aligned microcrystalline $\mathrm{RE}_{2} \mathrm{Fe}_{14} \mathrm{~B}$ is separated by a thin layer of the paramagnetic rare earth rich phase. The rare earth rich phase acts to magnetically decouple the grains and smooth grain boundaries to prevent reverse domain nucleation. In polymer-bonded magnets, the alloy is rapidly solidified in order to obtain particulate with a nanocrystalline microstructure, and then embedded within a polymer matrix. The fine grain structure pins domain walls. This chapter will describe fundamentals of the system and detail how it is processed to obtain the desired properties. Particular focus will be placed on processing through melt-spinning, as it is one of the primary processing routes utilized in this work.

\section{1 $-\mathrm{Nd}_{2} \mathrm{Fe}_{14} \mathrm{~B}$ discovery, structure, and intrinsic properties}

In 1984, Croat et al. reported the discovery of a new magnetic material with an energy product of $14 \mathrm{MGOe}$ [11]. They had been studying rapidly solidified $\mathrm{RE}-\mathrm{Fe}$ alloys, when an alloy with composition $\mathrm{Nd}_{0.135} \mathrm{Fe}_{0.817} \mathrm{~B}_{0.048}$ produced the best isotropic magnetic properties to that point. As Croat, Herbst et al. studied rapidly solidified materials, Sagawa, Fujimara et al. [12] investigated traditional powder metallurgy approaches to processing the material. They were able to produce a material with an energy product of $36 \mathrm{MGOe}$ from the composition $\mathrm{Nd}_{0.15} \mathrm{Fe}_{0.77} \mathrm{~B}_{0.08}$. In the following years, energy products as high as 56 MGOe were achieved by Rodewald et al. [13] through careful control of the composition and processing.

A number of attempts were made to determine the exact stoichiometry of the compound [14-16]. The accurate crystal structure and stoichiometry were finally determined by Herbst et al. through neutron powder diffraction studies [17, 18]. Figure 5 shows the crystal structure. 


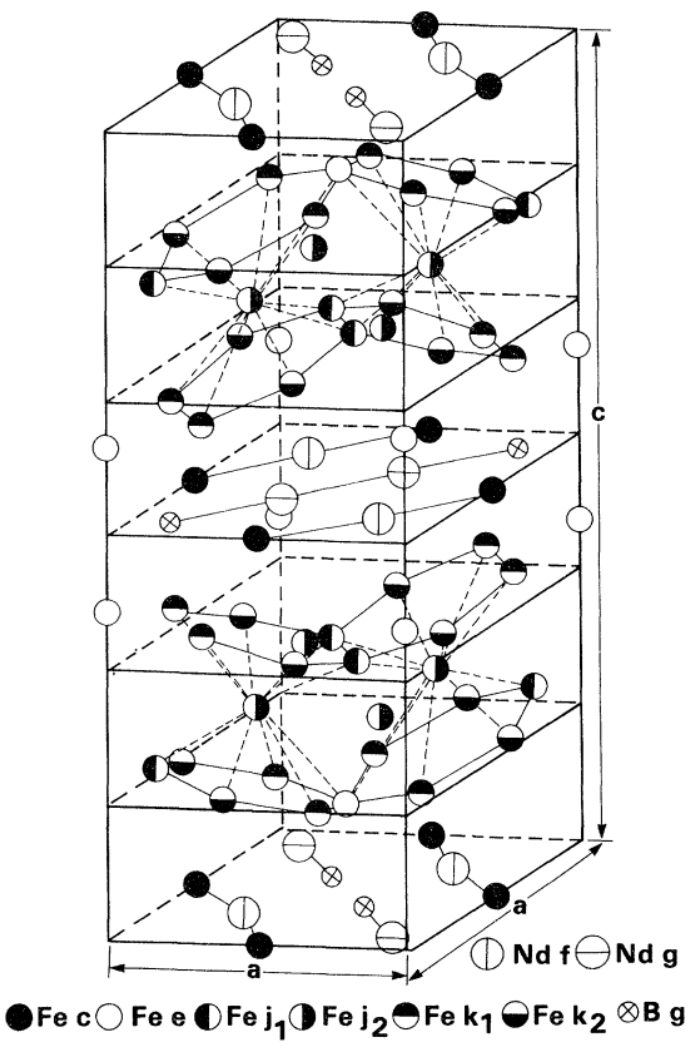

Figure 5 - Crystal structure of $\mathrm{Nd}_{2} \mathrm{Fe}_{14} \mathrm{~B}$ [19]

The structure displays space group $\mathrm{P} 4_{2} / \mathrm{mnm}$. It is a tetragonal structure with lattice parameters $\mathrm{a}=8.80 \AA$ and $\mathrm{c}=12.20 \AA$. There are 68 atoms ( 4 formula units) within the unit cell. This includes 2 crystallographically unique $\mathrm{Nd}$ sites, 6 unique $\mathrm{Fe}$ sites, and $1 \mathrm{~B}$ site. Figure 6 shows a calculated $\mathrm{Cu}-\mathrm{K}_{\alpha} \mathrm{x}$-ray diffraction pattern [19].

Within the structure, the magnetic moments of the $4 \mathrm{f}$ orbitals in the $\mathrm{Nd}$ align parallel to the $3 \mathrm{~d}$ orbitals in the Fe, resulting in a very high saturation magnetization ( $16 \mathrm{kG})[20]$. Additionally, the iron within the lattice forms a networked structure, effectively creating layers of iron within the lattice. From this order, the lattice displays very high uniaxial magnetocrystalline anisotropy. The high anisotropy allows for very high coercivities. Resulting from the high saturation magnetization and the coercivity achievable, the maximum possible energy product for the system is $65.6 \mathrm{MGOe}$. 


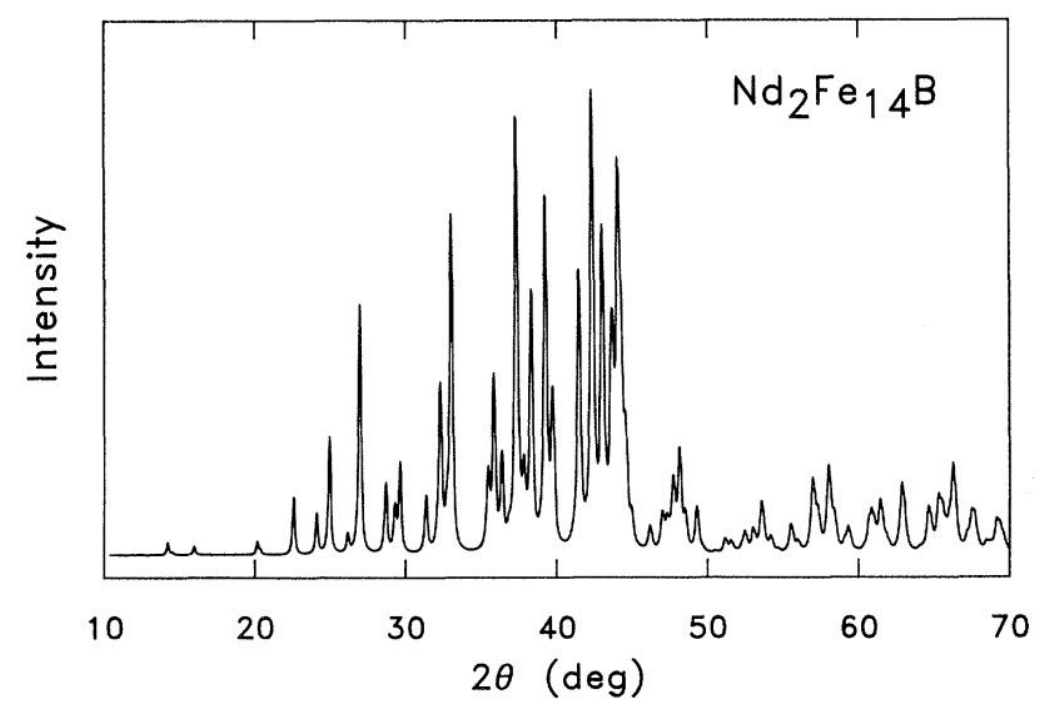

Figure 6 - Calculated Cu K- $\alpha$ diffraction pattern of $\mathrm{Nd}_{2} \mathrm{Fe}_{14} \mathrm{~B}$ [19]

\section{2 - Coercivity mechanisms}

One very important property of a permanent magnet is its ability to withstand demagnetization. Coercivity is the property that we attribute to the material in order to describe this behavior. There are a number of microstructural mechanisms that can lead to improved coercivity. All of these mechanisms utilize domain wall energy to prevent nucleation or hinder dislocation motion. One requirement for coercivity is the presence of anisotropy. Without it, the magnetization present in the material could simply rotate to be oriented in whatever direction was energetically favored by magnetostatic energy. There are two primary types of anisotropy that are important in dealing with permanent magnets: shape anisotropy and magnetocrystalline anisotropy. Shape anisotropy works by having a large aspect ratio within the material. By having this structure, the material will preferentially magnetize along the long direction of the particle. This is energetically favorable due to the presence of a smaller demagnetization factor along the length of the particle. This is the type of anisotropy that the Alnico class of magnets takes 
advantage of. It should be noted that, for this type of anisotropy to be effective, small particle sizes are required.

$\mathrm{Nd}_{2} \mathrm{Fe}_{14} \mathrm{~B}$, on the other hand, utilizes magnetocrystalline anisotropy. Magnetocrystalline anisotropy occurs when certain crystallographic directions require different energies to align the magnetic moments in that direction. If the difference in energy required to magnetize the easy axis is much lower than the energy needed to magnetize along the hard axis, the material has high magnetocrystalline anisotropy. Materials with high magnetocrystalline anisotropy are characterized by having a high anisotropy field, which is the theoretical coercivity of the material if coherent rotation were the only mechanism of domain motion. $\mathrm{Nd}_{2} \mathrm{Fe}_{14} \mathrm{~B}$ has an extremely high anisotropy field (73kOe [21]).

While coherent rotation is important in materials with low magnetocrystalline anisotropy, the coercivity in materials with high magnetocrystalline anisotropy is primarily affected by domain processes. Magnetization reversal in these materials is typically limited by either domain nucleation or domain growth.

In materials controlled by domain nucleation, sites in which reverse domains nucleate must be eliminated in order to maintain coercivity. These sites can be either chemical or physical defects. A soft magnetic material provides an easy starting point for a reverse domain, leading to low coercivity. Physical defects (typically irregularly shaped grain or phase boundaries) are also sites for demagnetizing fields. By eliminating these sites, coercivity can be greatly increased. This is often done by reducing grain size to minimize surface area per particle. The presence of secondary phases at grain boundaries can also help to isolate the particles and smooth grain boundaries. This is used in sintered $\mathrm{Nd}-\mathrm{Fe}-\mathrm{B}$ magnets.

A second method of improving coercivity is through domain wall pinning. By incorporating defects into the bulk of the material, domain wall motion can be reduced. Two methods are typically used to do this. The addition of small particles of a second, nonmagnetic, phase can interact with the domain walls, reducing total domain wall energy. The particles must be sized on the order of the domain wall 
thickness and dispersed throughout the structure. The second method is to reduce grain size. When no field is present, the critical grain size (D) at which only one domain will be present in a material is calculated as a function of saturation magnetization $\left(\mathrm{M}_{\mathrm{s}}\right)$ and domain wall energy $(\mathrm{y})$ :

$$
D=1.48 \mathrm{\gamma} /\left(4 \pi \mathrm{M}_{\mathrm{s}}\right)^{2}[22]
$$

When energy is added in the form of an applied field, the grain size must be smaller to maintain single domain status.

\section{3 - Isotropic vs. anisotropic magnets}

Since the crystal structure of $\mathrm{Nd}_{2} \mathrm{Fe}_{14} \mathrm{~B}$ is tetragonal and the system has an easy direction of magnetization along the c-axis, it is possible to create both isotropic and anisotropic bulk materials based on texturing of the microstructure. By having a magnetically anisotropic structure, energy product and remanence can be greatly improved over magnetically isotropic structures. One of the early models used to describe anisotropy was the Stoner-Wohlfarth model of rotational hysteresis [23]. The model is based on an aggregate of uniaxially anisotropic magnetic particles. In this model, the anisotropy energy is described as: $E_{a n}=K \sin ^{2} \theta$ where $E_{a n}$ is the anisotropy energy, $K$ is a material constant, and $\theta$ is the angle between the applied field and the easy axis of magnetization. The anisotropy energy can then be used to calculate the critical field necessary to saturate the particle and determine what the hysteresis loop would look like. Figure 7 shows the hysteresis loop for a single domain particle in which the easy axis is perpendicular to the applied field. Figure 8 shows the hysteresis loop for a particle in which the magnetization direction is anti-parallel to the applied field. Figure 9 shows the same thing for a particle in which the easy axis is at a different arbitrary angle to the applied field.

When all of the particles of the aggregate are taken into account, the hysteresis loop for the bulk will be a composite of all those of the individual particles. For example, if all of the particles were aligned parallel to the applied field, the bulk hysteresis loop would appear very similar to that shown in Figure 8, with the 
remanent magnetization being nearly equivalent to the saturation magnetization. If, however, the particles are randomly oriented, the resulting microstructure will be isotropic and have a hysteresis loop similar to the one shown below in Figure 10. In contrast to a completely anisotropic magnet, the isotropic magnet has a remanent magnetization that is half of the saturation magnetization. As a result, the maximum energy product is also reduced by at least a factor of 4 , due to the reduced remanence and non-rectangular demagnetization curve.
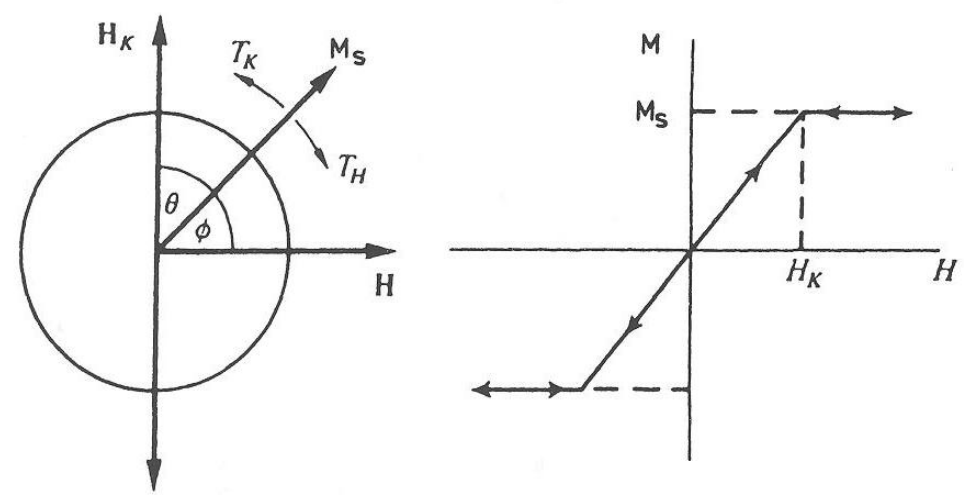

Figure 7 - Schematic of single domain particle with applied field perpendicular to the easy axis and the corresponding hysteresis loop [3, 23].

(a)
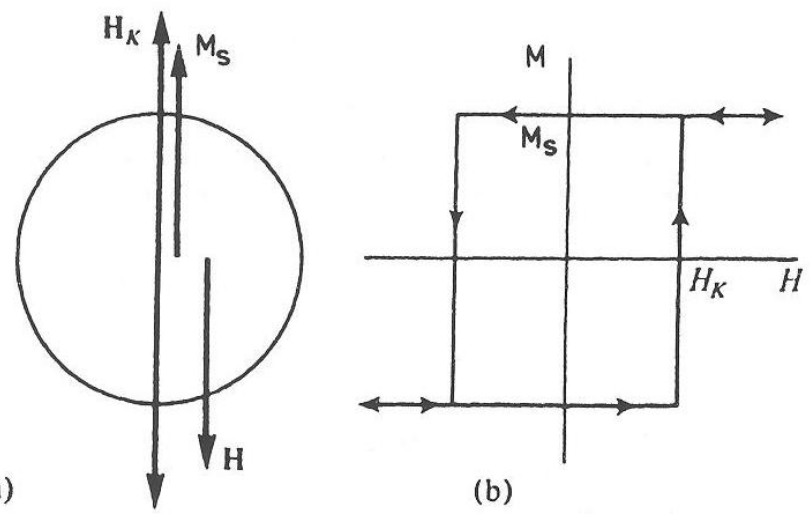

(b)

Figure 8 - Schematic of a single domain particle with applied field anti-parallel to the direction of magnetization and the corresponding hysteresis loop [3, 23]. 

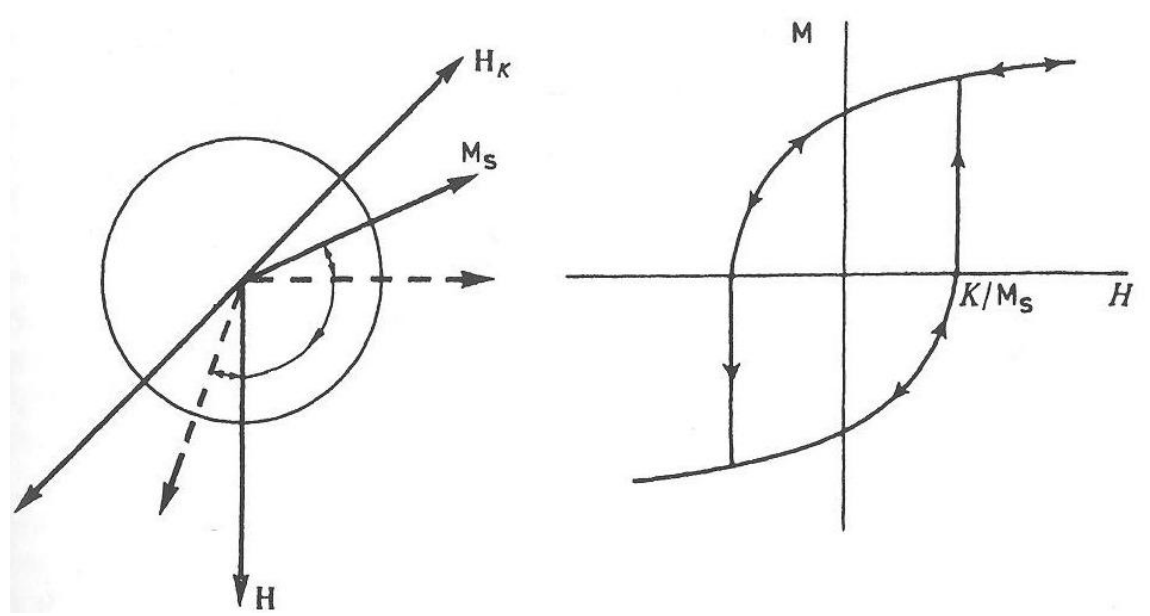

Figure 9 - Schematic of a single domain particle with the applied field at an arbitrary angle to the easy axis and the corresponding hysteresis loop $[3,23]$.

The Stoner-Wohlfarth model does an excellent job illustrating the difference between isotropic and anisotropic agglomerates of single domain particles. It does, however, have certain drawbacks. The model assumes that coherent domain rotation is the primary method of magnetic switching. However, in materials with high magnetocrystalline anisotropy, domain wall processes become the primary mode of magnetization change. One result is that the critical fields for domain switching are often smaller than those prescribed by Stoner and Wohlfarth.

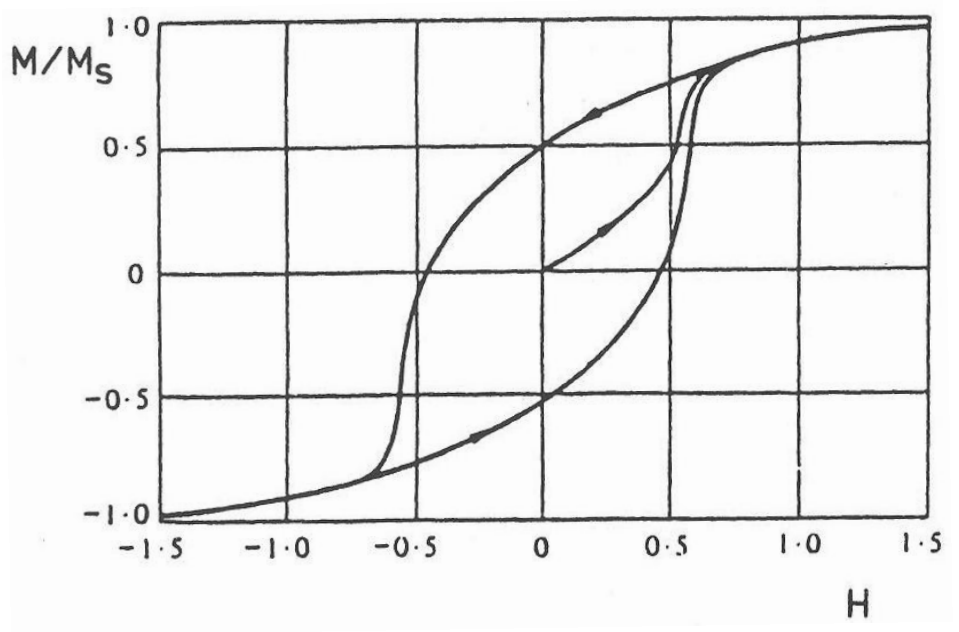

Figure 10 - Hysteresis loop for isotropic magnet [3, 23]. 


\section{4 - Processing sintered magnets}

Aligned and sintered magnets represent the strongest magnets available to date. The general processing technique involves the following steps: generate magnet particulate, align the particulate, press, and sinter.

Generation of the magnetic particulate is typically done by casting a block of the alloy, followed by milling until a particle size of roughly $1-3 \mu \mathrm{m}$ is achieved [12]. One goal of the milling process is to achieve single crystal particles, so that in the presence of an applied magnetic field, the particulate will align so the c-axis aligns with the field. The particulate is then placed in a die and aligned under a magnetic field on the order of 1 Tesla. While in the field, the particulate is pressed into a green compact. Typical pressures used are on the order of $200 \mathrm{MPa}$. The green compact is then sintered at temperatures on the order of $1000-1200^{\circ} \mathrm{C}$. A lower temperature heat treatment is then done in order to achieve proper grain boundary phases [24].

Since the rare earth elements readily oxidize, much emphasis is placed on protecting the material. This is especially true when the alloy is in particulate form since the surface area is increased. Because of this, all processing is done in a carefully controlled environment (typically an argon atmosphere is utilized).

Alloy selection is also an important factor in the magnet design process. Since the primary coercivity mechanism in these types of magnets is prevention of reverse domain nucleation, a method is needed for smoothing out grain boundaries. In the $\mathrm{Nd}-\mathrm{Fe}-\mathrm{B}$ system, there is a low melting ternary eutectic phase present at $665^{\circ} \mathrm{C}$ between $\mathrm{Nd}_{2} \mathrm{Fe}_{14} \mathrm{~B}$, a Nd-rich phase, and $\mathrm{Nd}_{1.1} \mathrm{Fe}_{4} \mathrm{~B}_{4}$. Phase diagrams are shown below in Figure 11 and Figure 12. 


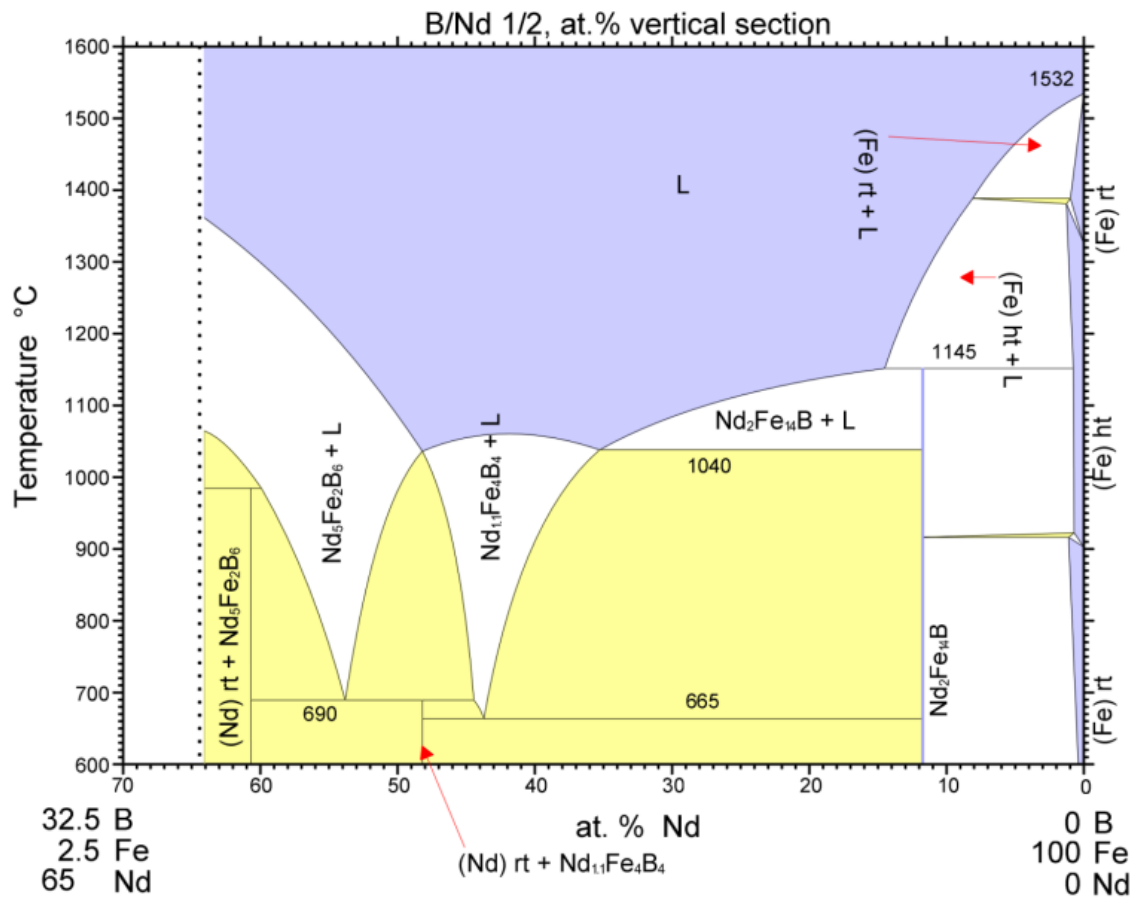

Figure 11 - Pseudo-binary Nd-Fe-B phase diagram [25]

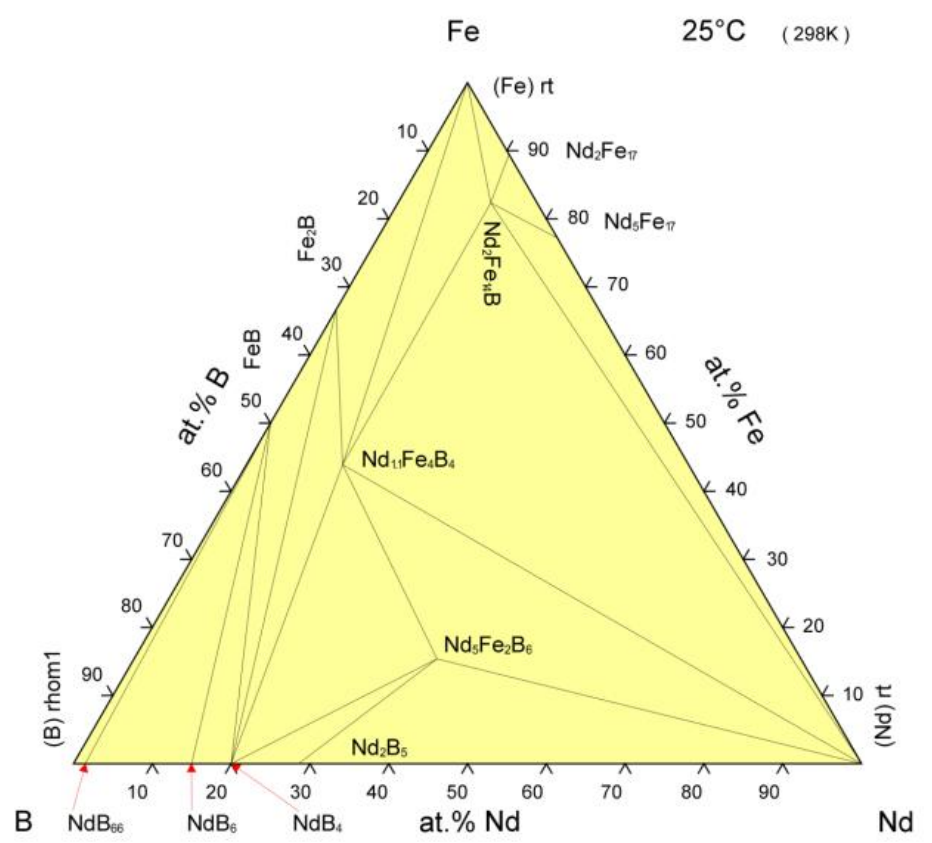

(c) ASM International 2009. Diagram No. 200480

Figure 12 - Nd-Fe-B ternary phase diagram at room temperature [26] 
By adding excess $\mathrm{Nd}$ to the system, a small fraction of liquid can be obtained at this lower temperature. During the post-sintering anneal, this liquid phase serves the purpose of rounding sharp corners in the grains and decoupling the individual crystals, preventing domains from crossing from one particle to the next. Figure 13 shows a typical desirable microstructure. It is characterized by grains on the order of $10 \mu \mathrm{m}$ or less, surrounded by a thin layer of a secondary rare earth rich phase.

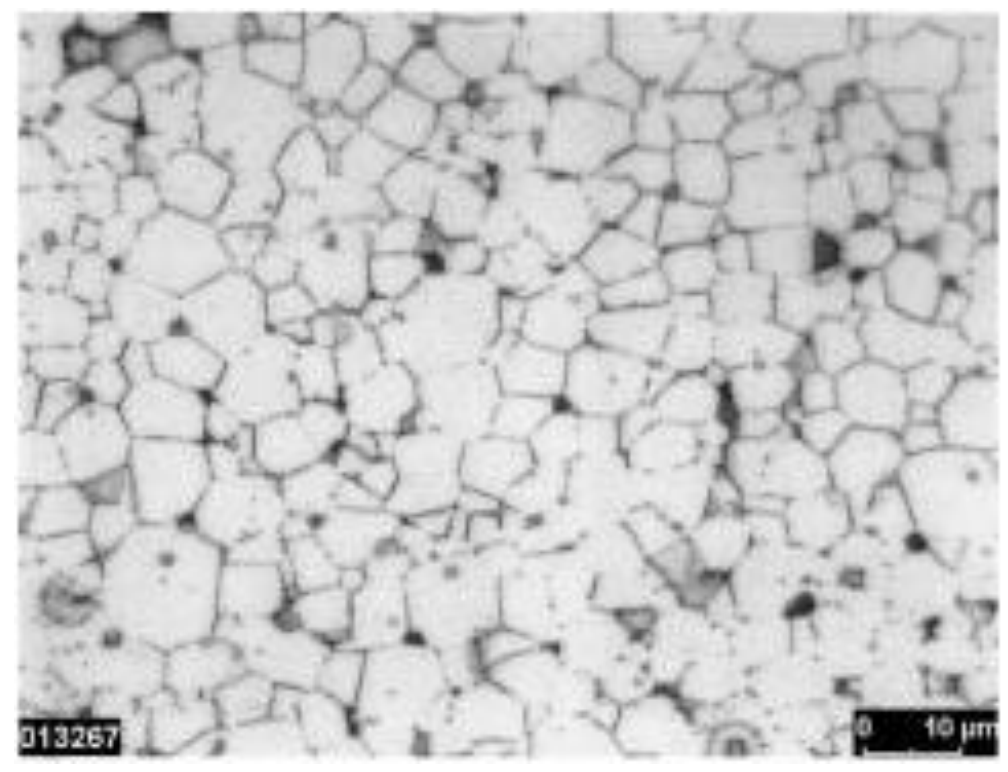

Figure 13 - Microstructure of sintered Nd-Fe-B magnet [13]

\section{5 - Processing bonded magnets}

In a polymer-bonded magnet, magnetic particulate is embedded within a polymer matrix. The particulate is generally processed using rapid solidification techniques, such as melt-spinning [27] or high pressure gas atomization. In the past, melt-spinning has been the commercial option of choice. Melt-spinning is a process where a molten metal is impinged on a rapidly rotating wheel and a thin ribbon of the alloy is formed. Often, $\mathrm{Cu}$ or Mo is used for the wheel surface. Rapid solidification allows for the formation of extremely fine-grained, or even glassy material, 
depending on alloying and processing parameters. This is achieved through rapid cooling rates, on the order of $10^{4}-10^{7} \mathrm{C} /$ second [28].

This process is very helpful in the generation of particulate for bonded magnet applications. By rapidly solidifying the material, microsegregation from the peritectic and eutectic reactions in the Nd-Fe-B system can be reduced [29].

Additionally, the fine-grained microstructure effectively pins magnetic domain walls, providing a material with good coercivity.

In practice, the melt is typically over-quenched, resulting in a glassy (or partially glassy, partially crystalline) structure. The material is then heat treated to crystallize the amorphous material and achieve a nanometric polycrystalline microstructure. While a single domain particle in Nd-Fe-B is calculated to be $0.3 \mu \mathrm{m}$ [22], the ideal grain diameter to maximize coercivity is on the order of $20-50 \mathrm{~nm}$. An example of the desired microstructure can be seen below in Figure 14. The ribbons are then coarsely ground to particle sizes on the order of 50-150 $\mu \mathrm{m}$ in diameter. Next, they are blended with a polymer and either compression molded or injection molded. The polymer provides several benefits. Since each particle is embedded within the polymer, corrosion resistance can be improved. Additionally, the polymer provides a matrix with high toughness, reducing cracking of the final product. The ability to mold the polymer allows for complex geometries to be used without significant wasted material.

In some cases, greater physical strength than a polymer can provide is desired. Attempts have been made at creating a "metal-bonded" magnet, where a soft metal, such as Al or $\mathrm{Zn}$ is used as the matrix phase [30]. In these attempts, isotropic, nanocrystalline material was used. 


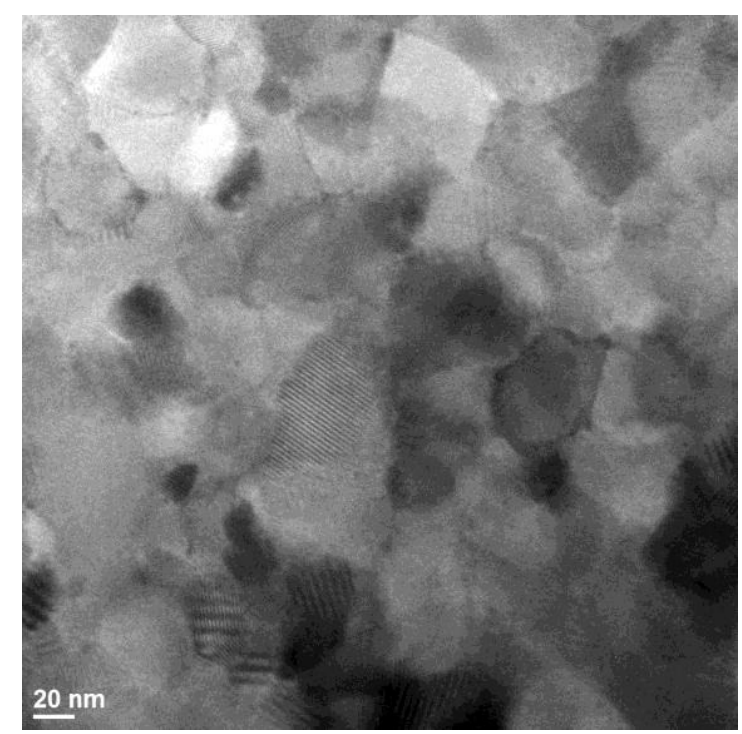

Figure 14 - TEM micrograph of rapidly solidified $\mathrm{Nd}_{0.8} \mathrm{Y}_{0.8} \mathrm{Dy}_{0.4} \mathrm{Fe}_{14} \mathrm{~B}+3$ at. \% TiC

By utilizing polymer-bonded magnets, processing costs can be drastically reduced, but not without drawbacks. The biggest disadvantage of this processing method is that with most bonded magnets, the resulting magnet is isotropic in nature, reducing the maximum energy product achievable. Attempts have been made to form anisotropic nanocrystalline microstructures suitable for bonded magnet applications. This will be further described in section 3.6.

Another drawback of using polymer-bonded magnets is that the volume of magnetic material in the final product is reduced from that obtained in sintered magnets. In sintered magnets, the thin layer of intergranular phase is the only nonmagnetic portion, resulting in upwards of 85 vol\% magnetic material [31]. In bonded magnets, a greater volume of polymer is needed. Extrusion bonded magnets require roughly 40 vol\% polymer, while compression bonded magnets require $30 \mathrm{vol} \%$. As a result, even an anisotropic bonded magnet will display reduced remanence and energy product from its sintered magnet counterpart, proportional to the volume fraction of magnetic material. Figure 15 shows how properties are affected by this. 


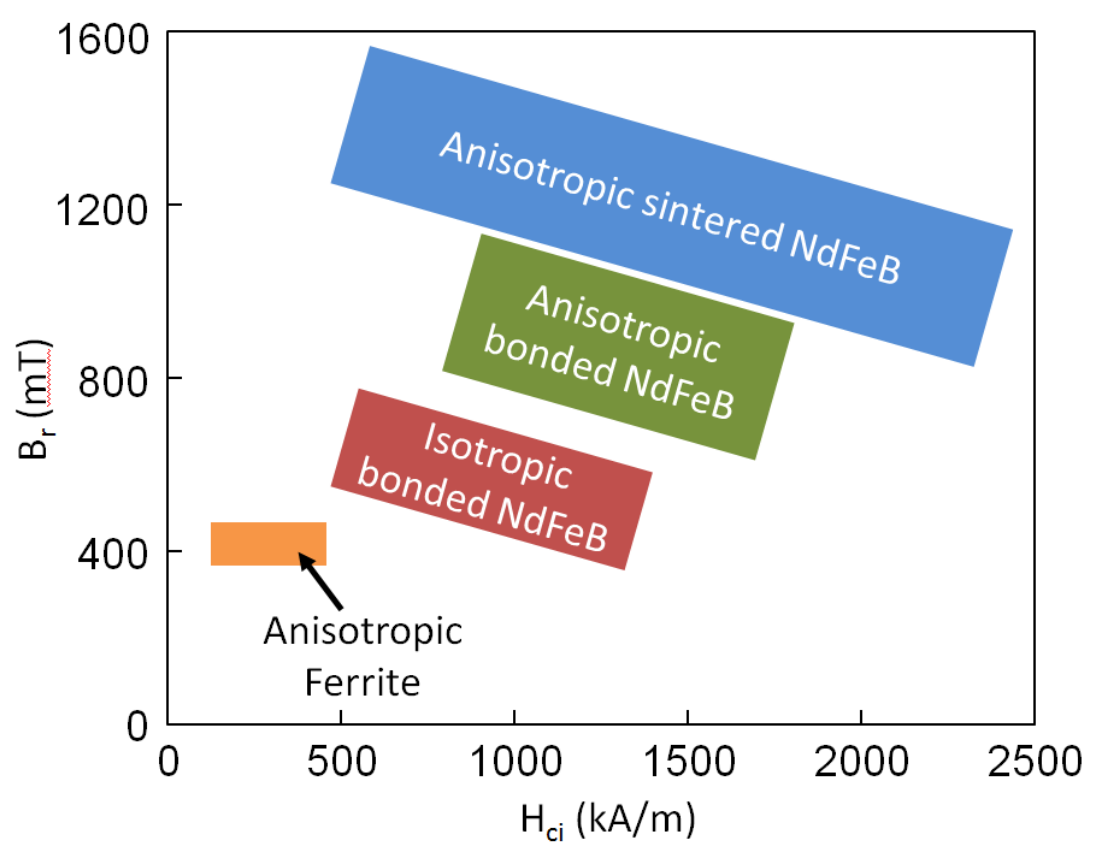

Figure 15 - Remanence and coercivity resulting from various processing methods (adapted from [5])

\section{6 - Other processing techniques}

A number of other processing techniques have been employed in order to achieve desirable microstructures consisting of aligned nanocrystalline grains. The two methods that have been realized on a large scale are hydrogenation, disproportionation, desorption, and recombination (HDDR) and the die-upset process. Recently, work involving the use of surfactant assisted ball milling has been done to allow for greater variation in compositions, but has only been attempted on a small scale.

The HDDR process was developed by Takeshita and Nakayama in the late 1980's [32, 33]. In the process, magnet particulate is placed in a high pressure hydrogen atmosphere and heated. The hydrogen reacts with the system, turning the $\mathrm{Nd}_{2} \mathrm{Fe}_{14} \mathrm{~B}$ into a mixture of $\mathrm{Nd}_{2} \mathrm{H}_{3}, \mathrm{Fe}_{2} \mathrm{~B}$ and Fe. While maintaining temperature, the atmosphere is changed to a vacuum, and the hydrogen is withdrawn. The remaining material then reforms into $\mathrm{Nd}_{2} \mathrm{Fe}_{14} \mathrm{~B}$, but with a smaller grain size. The grains also 
remain aligned since the reformed structure templates to the prior structure. Effectively, additional grain boundaries are added to the microstructure.

Figure 16 shows an example thermal and atmosphere profile typical of the HDDR process. Figure 17 shows the resulting microstructure after HDDR is completed. By carefully controlling parameters, HDDR can be used to form anisotropic material for use in bonded magnets.

One of the major drawbacks of this process, though, is that the resulting structure displays decreased thermal stability [34-36]. Because of this, operating temperatures exceeding approximately $80^{\circ} \mathrm{C}$ result in significant magnetic property degradation. Since operating temperatures exceeding $150^{\circ} \mathrm{C}$ are desired, this method is not satisfactory for traction motor applications.

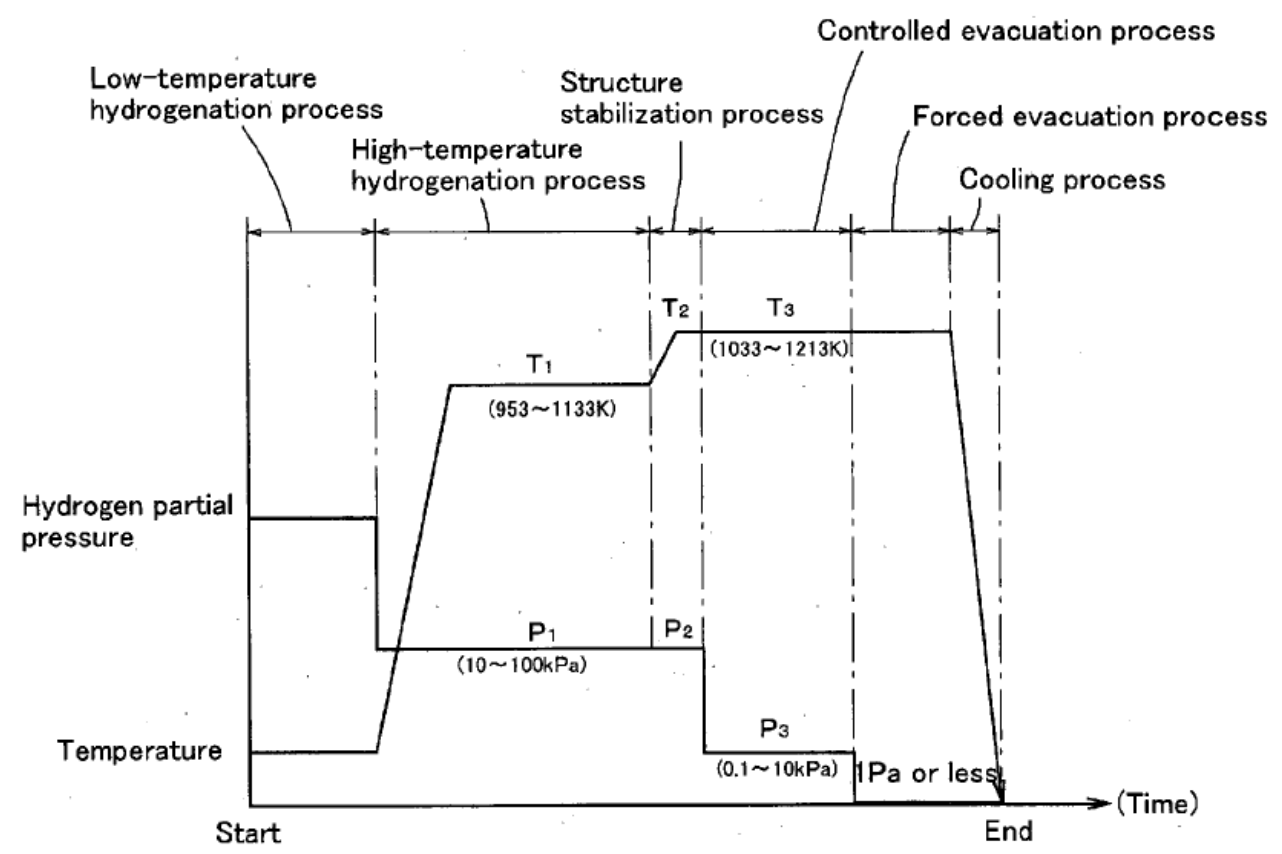

Figure 16 - Example of a HDDR thermal and atmospheric profile [37] 


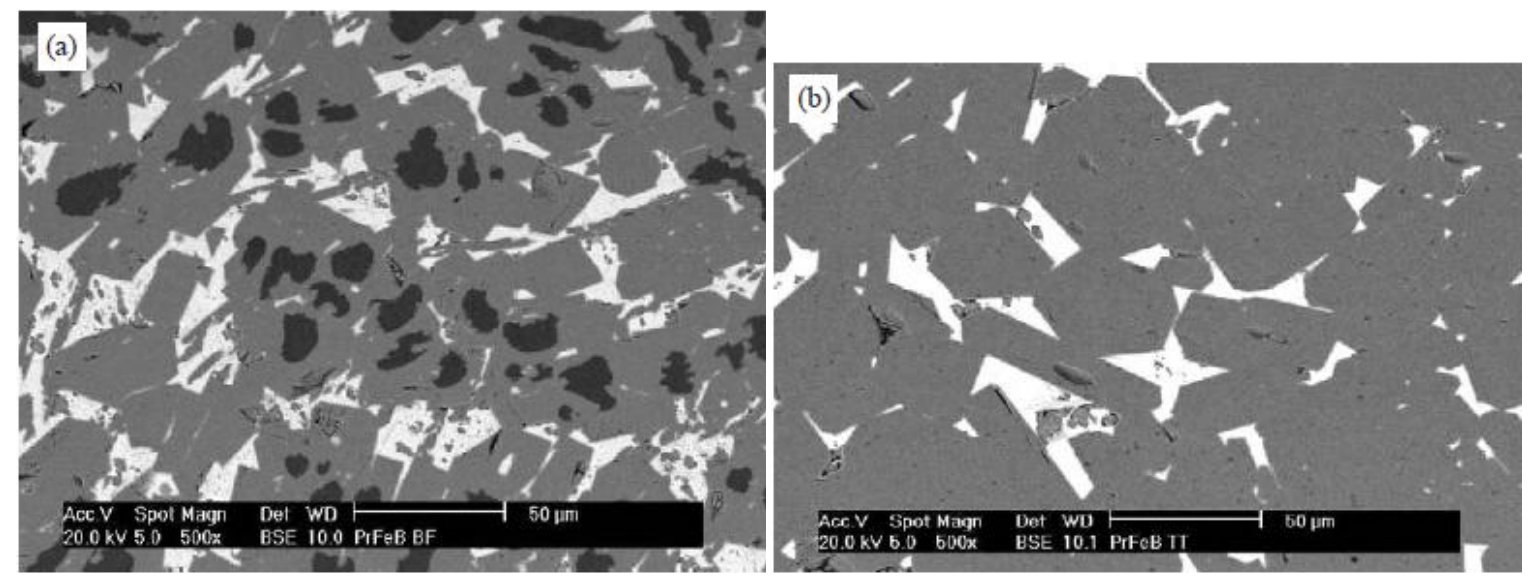

Figure 17 - Microstructure of $\operatorname{Pr}_{14} \mathrm{Fe}_{80} \mathrm{~B}_{6}$ (a) after hydrogen disproportionation and (b) after recombination [38]

Die upset is another method for creating fine anisotropic microstructure. The method is based on observations made by Kamb in 1959-1961 [39, 40]. The research was a study of crystallization in geological formations, where the presence of non-hydrostatic pressure existed. It was determined that the favored direction of growth was such that "the weakest axis is aligned parallel to the axis of greatest compressive stress."

$$
\mu_{z}^{\|}-\mu_{z}^{\perp}=V_{0} \cdot \frac{1}{2}\left(s_{11}-s_{33}+2 s_{12}-2 s_{13}\right) \cdot\left(P_{z}-P_{x}\right)^{2}
$$

where $\mu_{z}^{\|}$is the chemical potential across the surface perpendicular to the $\mathrm{c}$-axis when the compressive stress is applied parallel to the c-axis, $\mu_{z}^{\perp}$ is the chemical potential of the surface perpendicular to the $c$-axis when the compressive stress is applied perpendicular to the $\mathrm{c}$-axis, $\mathrm{V}_{\mathrm{o}}$ is the specific volume, $\mathrm{s}_{\mathrm{ij}}$ are the crystal compliance coefficients, and $\mathrm{P}$ is the applied pressure along either the $\mathrm{z}$ or $\mathrm{x}$ axes.

This can be utilized since the a-axis in $\mathrm{Nd}_{2} \mathrm{Fe}_{14} \mathrm{~B}$ has a higher elastic constant than the c-axis. As a result, when uniaxial pressure is applied, grains will grow such that the c-axis will be parallel to the direction of applied compressive stress. Early applications of this phenomenon simply involved hot pressing near- 
stoichiometric melt-spun ribbon into compacts. This method is able to achieve roughly $10 \%$ alignment of grains. Later, R.W. Lee at General Motors Research Laboratories created what is known as the die-upset method [41, 42]. In this method, melt-spun ribbon is first hot pressed into a compact, then placed in a larger die and effectively hot forged. The texturing saturates at roughly $60-70 \%$ compressive deformation [43], resulting in approximately $75 \%$ alignment. Figure 18 below shows a typical resulting microstructure.

$\mathrm{Li}$ and Graham [43] have proposed a mechanism for the alignment of the particles. They describe a grain boundary-type diffusion, in which the unfavorably oriented surfaces are dissolved into the liquid boundary phase and precipitated onto favorably oriented grains. In this model, liquid is required not only to allow hot deformation, but also to provide atomic transport. Many studies have been done on the effects of strain rate [44], rare earth content [45, 46], and temperature [41, 42] on hot workability of the compact.

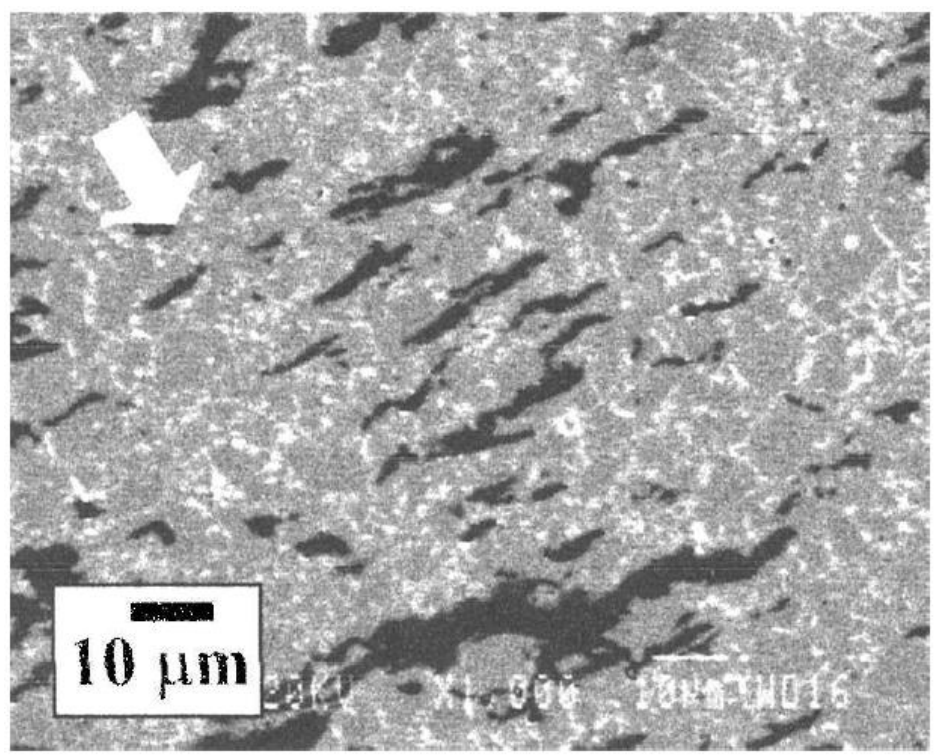

Figure 18 - Microstructure of hot forged $\mathrm{Nd}_{11.8} \mathrm{Fe}_{80.6} \mathrm{~B}_{6} \mathrm{Cu}_{1.5}$. he white arrow indicates forging direction. [47]

While the die upset method produces magnet particulate with very good properties and was commercially produced for a time, the material is no longer in 
production. The final product was very similar to that of a sintered magnet, but the cost of production exceeded that of sintered magnets.

Other methods stemming from the die upset process have been proposed as possible processing routes to induce texture within a nanocrystalline structure. One of the methods involves first creating an amorphous structure then crystallizing under uniaxial pressure. This has been attempted for RE-lean magnetic materials for use in nanocomposite exchange-spring magnets by Liu et al. [46] and Wu et al. [48]. Both groups found that texture was moderately increased when crystallization occurred when exposed to very high uniaxial pressure (on the order of $1 \mathrm{GPa}$ ).

A more recent method of inducing texture through a process known as surfactant-assisted ball milling has been attempted by several groups [49-53]. In this process, isotropic (or large-grained) particulate, milling balls, and an organic surfactant (often oleic acid) is placed within a ball mill jar and high energy ball-milled. After milling, the surfactant is removed and a (hopefully) textured set of nanoparticles of the desired composition is obtained. An additional benefit is that composition can be easily tailored. It allows for creation of a nanoscale, Fe-rich material to be formed. This is critical in the creation of exchange-spring magnets.

In the Sm-Co system, this process appears to produce anisotropic flakes displaying good coercivity (on the order of several kOe). However, in the $\mathrm{Nd}-\mathrm{Fe}-\mathrm{B}$ system, the observed coercivities are lower ( $\sim 1 \mathrm{kOe}$ at a temperature of $40 \mathrm{~K})$. Figure 19 shows the morphology of the particulate formed in this process. It can easily be seen that the surface area to volume ratio is very high for these systems. Due to this, one major issue with this process is oxidation of the particulate. Another is the ability to effectively consolidate the particulate into a bulk material without significantly degrading the magnetic properties.

Not much work has been done on feasibility studies to see if this process could be commercially viable. However, it is unlikely that this would easily ramp to a commercial scale due to the potential yields of high energy ball milling. Currently, the highest capacity high energy ball mills are only capable of processing up to about $250 \mathrm{~g}$ per batch [54]. The particulate observed so far has required milling times on 
the order of 10 hours to create a sufficient degree of texture. This would likely make the cost prohibitively high.

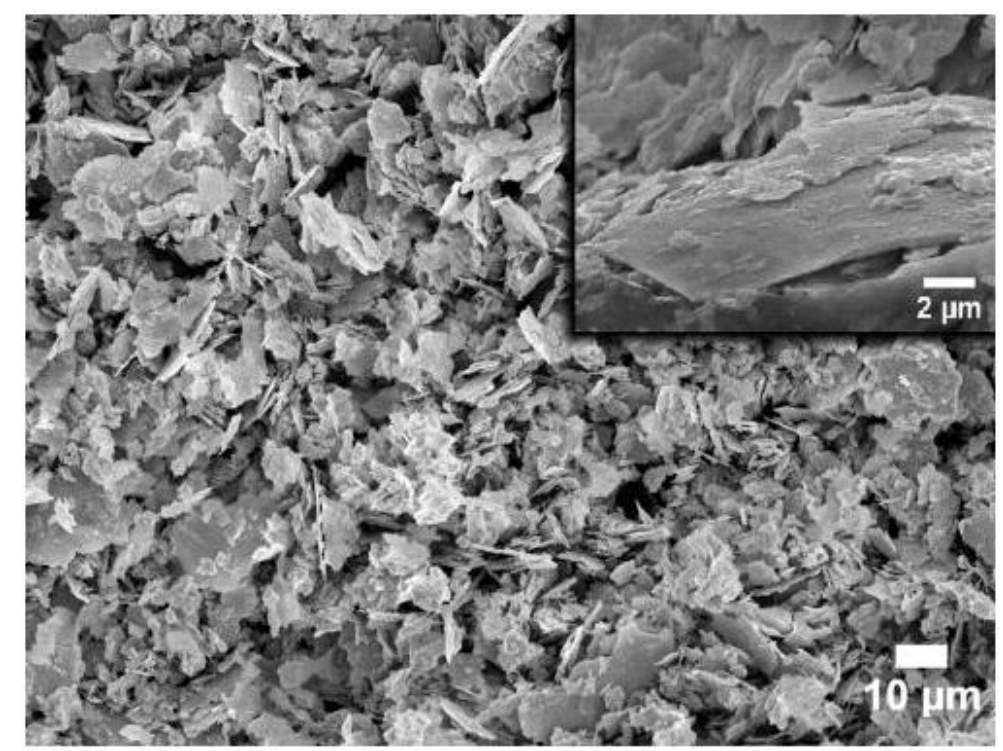

Figure 19 - Particle morphology of surfactant-assisted, ball-milled NdFeB [50].

\section{7 - Alloying considerations}

While the $\mathrm{RE}_{2} \mathrm{Fe}_{14} \mathrm{~B}$ systems are relatively complex, alloying is often done to modify the properties of the final product. Alloying additions can be broken into a few general categories: substitution in the 2-14-1 phase, modification of the grain boundary phase, or grain refinement. A fourth category to alloy selection can be made, though it does not necessarily require addition of further elementsmodification of the ratios of the elements in the base alloy.

By substituting in the 2-14-1 phase, itself, intrinsic properties are generally modified. While $\mathrm{Nd}_{2} \mathrm{Fe}_{14} \mathrm{~B}$ shows the highest saturation magnetization, its temperature performance is relatively poor, with coercivity dropping precipitously with increasing temperature. To combat this, Dy is often substituted for a portion of the $\mathrm{Nd}$. Dy shows a higher anisotropy field than $\mathrm{Nd}$, resulting in higher coercivity at elevated temperatures. However, in $\mathrm{Dy}_{2} \mathrm{Fe}_{14} \mathrm{~B}$ the moment of the Dy atoms aligns anti-parallel to the Fe atoms, resulting in a lowered remanent magnetization [19]. Tb 
can also serves the same function as Dy. However, $\mathrm{Tb}$ is not often used due to its high cost. Another substitution often made in high temperature alloys is Co for a portion of the Fe. As $x$ is increased from 0 to 14 in $\mathrm{Nd}_{2} \mathrm{Fe}_{14-x} \mathrm{Co}_{x} \mathrm{~B}$, Curie temperature is increased from $585 \mathrm{~K}$ to approximately $1000 \mathrm{~K}$. However, both anisotropy field and saturation magnetization are decreased as the material transitions from uniaxial anisotropy to basal plane anisotropy. Many other elements have been used as substitutional alloying additions, including $\mathrm{Ga}, \mathrm{Ni}, \mathrm{Si}, \mathrm{Al}, \mathrm{Cr}$, and $\mathrm{V}$ [19], but have been found to have either minimal or deleterious effects on magnetic properties.

A second class of alloying additions serves the function of modifying the grain boundary phase. These additions are typically used in either rapidly solidified materials for bonded magnet application, or in die-upset applications. The addition of copper has been of particular interest for die-upset applications. It has been determined that the grain boundary phase is changed from the Fe-RE eutectic to the $\mathrm{Cu}-\mathrm{RE}$ eutectic. This allows for lower temperature pressing due to the reduced temperature of the eutectic reaction $\left(\sim 520^{\circ} \mathrm{C}\right.$ vs. $\left.665^{\circ} \mathrm{C}\right)$. Additionally, it has been determined that the Cu-Nd liquid allows for increased transport of the 2-14-1 phase, resulting in greater deformation capability [55]. It has also been reported that Cu can greatly improve coercivity melt-spun materials [56].

The third class of alloying additions consists of materials added with the intention of minimizing grain size or forming amorphous material. These compounds are important in bonded magnet applications to improve uniformity of the nanocrystalline structure and improve coercivity. Brannagan, McCallum, Kramer, et al. have researched the effects of adding transition metal carbides to the alloy [5760]. They determined that $\mathrm{TiC}$ and $\mathrm{ZrC}$ were the most effective at maintaining grain size and improving coercivity. In practice, the carbides are rejected from the 2-14-1 particles to the grain boundaries, particularly triple junctions. As the material is rejected, it interferes with continued grain growth [61].

A fourth category of alloying is alteration of the ratio of the base alloy elements. This will change the relative phase assemblage of the material and can be done for a number of different reasons. For example, excess rare earth elements are 
used in sintered magnets to increase the fraction of the $\mathrm{Nd}$-rich grain boundary phase. This will improve the sintered density as well as improve the coercivity of the material. It also reduces the fraction of free $\alpha-F e$ and $R E_{2} F_{17}$, phases that can be detrimental to high temperature properties.

In certain cases, excess Fe will be added with the intention of forming either $\mathrm{Fe}_{2} \mathrm{~B}$ or $\mathrm{\alpha}-\mathrm{Fe}$. These are both soft magnetic phases, but display higher saturation magnetization than the $\mathrm{Nd}_{2} \mathrm{Fe}_{14} \mathrm{~B}$ structure. Typically the two-phase structure would be expected to display magnetic behavior that is simply an addition of the two hysteresis loops proportional to the volume of each phase. Figure 20b shows a schematic of the expected hysteresis loop. However, if the distribution of the two phases can controlled such that the soft phase will coat the hard phase with a layer less than approximately $10 \mathrm{~nm}$ thick, the material will behave as a single magnetic phase with improved saturation magnetization, while still maintaining a significant portion of the coercivity of the hard phase. This idea was proposed by Kneller and Hawig [62], who coined the term "exchange-spring magnet" to describe this phenomenon.

(a)

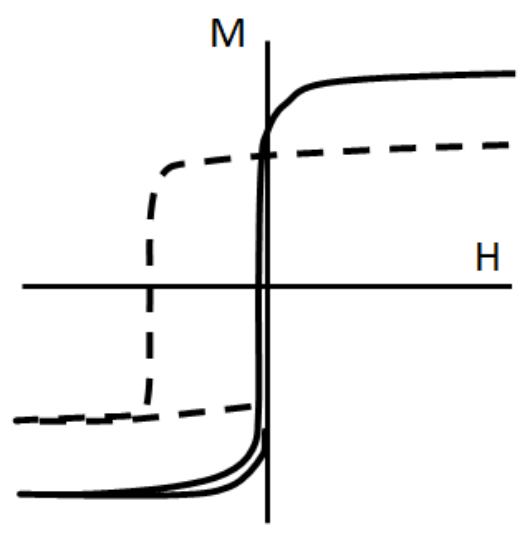

(b)

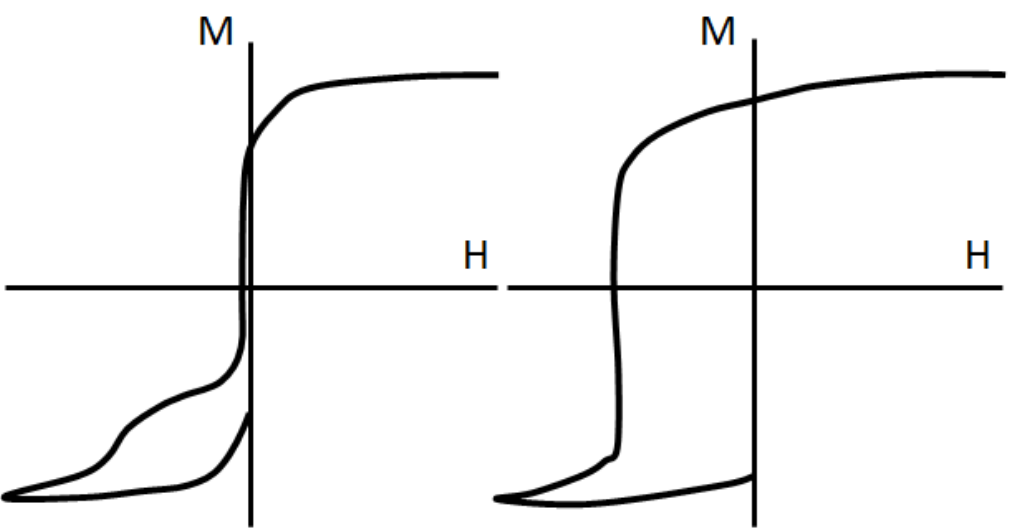

Figure 20 - Schematic hysteresis loops for a composite of a soft and a hard magnetic material. The hysteresis behavior of the two materials when separate is shown in (a). In (b) the hysteresis behavior of a composite of the two materials when fabricated in a coarse structure is shown. When the structure is formed on a fine enough scale (c), enhancement of the coercivity, remanence and energy product is seen. 
Isotropic exchange spring magnets have been successfully realized through melt-spinning, followed by either polymer-bonding $[63,64]$ or spark plasma sintering [65]. However, a challenge still exists to successfully create these in an anisotropic form. Attempts at die-upset [66, 67], thick film synthesis [68], chemical deposition [69], hot extrusion [70], and surfactant-assisted high energy ball milling [50] have all been attempted with very limited success. In each case, either the spacing is insufficiently small or the processing route is no longer viable with the alloy.

\section{8 - Solidification considerations}

In many of the processes described above, solidification microstructures play an important role. Further understanding of how the solidification process occurs is often helpful in determining how to form a desired microstructure. Solidification science is a broad field, with many factors affecting the behavior of the solidifying species [71]. It is a mix of thermodynamics, kinetics, heat transfer, and chemistry, among others. Since the work presented here focuses on rapidly solidified alloys, we will focus on the factors affected by rapid solidification of multicomponent systems.

One of the primary features of rapid solidification is the ability to obtain highly non-equilibrium states. Boettinger and Coriell have created a hierarchy of equilibrium states [72] (shown below). 
I. Full Diffusional Equilibrium

- No chemical potential gradients (phase compositions are uniform)

- No temperature gradients

- Lever rule applies

II. Local Interfacial Equilibrium

- Phase diagram gives compositions and temperatures only at liquid/solid interface

- Corrections made for interface curvature (Gibbs-Thomson effect)

III. Metastable Local Interfacial Equilibrium

- Stable phase cannot nucleate or grow sufficiently fast

- Metastable phase diagram (true thermodynamic phase diagram) gives the interface conditions

IV. Interfacial Non-Equilibrium

- Phase diagram fails at the interface

- Chemical potentials are not equal at the interface

- Free energy functions of phases still lead to criteria which predict impossible reactions

As the solidification rate/undercooling is increased lower positions on the hierarchy are achieved. Full diffusional equilibrium (I) is typically only obtained during very long term annealing treatments. Local interfacial equilibrium (II), while not truly obtained in practice, serves as the basis for many of the solidification models (such as the Scheil model, which assumes local equilibrium and no diffusion in the solid). It can serve as a very good approximation under many circumstances. Metastable local interfacial equilibrium (III) is what is truly seen in practice during conventional solidification processes [73]. Interfacial non-equilibrium (IV) is typically only achieved during rapid solidification processes.

During solidification of alloys, assuming local equilibrium, solute will pile up in front of the growing interface. As solute builds up in front of the progressing interface, the local composition will be increased in the solute. This will alter the local liquidus and solidus temperatures (though it should be noted that, with rapid solidification, the interface temperatures and compositions of the solid and liquid can vary significantly from that predicted by the equilibrium phase diagram). The thermal gradient imposed on the structure can dip below the local liquidus temperature, 
resulting in constitutional undercooling. The presence of this undercooling creates instability in the interface and the formation of dendritic or cellular structures.

Since the structures desired for permanent magnets are typically very fine, rapid solidification is used to refine the grains by increasing the solidification front velocity through a greater imposed thermal gradient. By increasing the velocity, greater concentrations of solute are pushed directly ahead of the interface, further increasing the constitutional undercooling and the driving force for formation of fine cellular and dendritic structures. If local equilibrium and zero diffusion within the solid are assumed, the stable perturbation wavelength is inversely proportional to the square root of the interfacial velocity [71]. However, in rapid solidification local equilibrium can break down if the front velocity exceeds the diffusion rate of the solute in the liquid.

For local equilibrium to be present, the chemical potentials of the solid and liquid in each species are equal, while there is a composition gradient over the interface. Interfacial non-equilibrium is reached when the solidification velocity is high enough that the chemical potential for each species is no longer equal in the solid and liquid. This means that the concentration of solute in the solid is increased beyond equilibrium conditions, a phenomenon known as solute trapping [29,74]. For extreme cases, the velocity will exceed the diffusion rates allowing for the formation of long range order (i.e. crystalline material), resulting in formation of an amorphous material. 


\section{Chapter 4 - The effects of applied uniaxial stress during devitrification on microstructure and texture}

Creation of anisotropic material for use in bonded magnet applications poses a number of engineering challenges. The particulate, itself, must display good magnetic properties prior to consolidation. In traditional rapidly solidified magnet materials, this is achieved by the creation of a nanometric grain size. However, most materials displaying good properties are nearly isotropic. In order to achieve an anisotropic structure, modification of how the material is processed is required.

One processing route to obtain nanometric textured material is to start with a solid material and induce texture. The die upset process (described in section 3.6) does this by utilizing uniaxial pressure to induce texture in nanocrystalline bulk materials. In order to effectively induce texture, however, the method requires a liquid phase to be present for the unfavorably oriented grains to dissolve in and to transport atoms to the favorably oriented grains for reprecipitation. This presents a problem for alloy systems containing significant amounts of heavy rare earth elements (e.g. Y and Dy), since the solidus temperature of the alloy can be increased upwards of $800^{\circ} \mathrm{C}$. Utilizing these temperatures for die upsetting would likely result in excessive grain growth and reduction of magnetic coercivity. One method to avoid the excessive grain growth is to produce an amorphous precursor and induce the texture upon crystallization. Grain growth can be further suppressed through the addition of a secondary phase which can act as a Zener pinning precipitate. TiC can serve as an effective grain pinning phase. Additionally, the presence of $\mathrm{TiC}$ can increase the glass formability of the alloy, resulting in easier production of the precursor material.

The work presented in this chapter investigates the effects of applied uniaxial pressure during devitrification on microstructure and texture of mostly amorphous $\mathrm{MRE}_{2} \mathrm{Fe}_{14} \mathrm{~B}+\mathrm{TiC}$ melt-spun ribbon. 


\section{1 - Experimental Methods}

Ingots ( 10g in size) of composition $\mathrm{Nd}_{0.8} \mathrm{Y}_{0.8} \mathrm{Dy}_{0.4} \mathrm{Fe}_{14} \mathrm{~B}+3$ at. \% TiC were created for melt-spinning feed stock. The constituent elements were first weighed to the proper proportions, then arc melted into a single button on a watercooled copper hearth using a non-consumable tungsten electrode. In the arc melting chamber, the buttons were re-melted at least twice to aid in mixing of the elements.

Melt-spinning was then used to obtain rapidly solidified ribbons for a mostly-amorphous precursor material used for crystallization from the amorphous state. A $10 \mathrm{~g}$ button of the desired alloy (created as described above) was placed in a quartz crucible with a $0.8 \mathrm{~mm}$ diameter opening. The ingot was then inductively heated to approximately $1400^{\circ} \mathrm{C}$ (approximately $150^{\circ} \mathrm{C}$ above the alloy's liquidus temperature). The melt was then ejected onto a rotating copper wheel with a tangential surface velocity of $25 \mathrm{~m} / \mathrm{s}$. The setup can be seen below in Figure 21 . The atmosphere of the chamber was maintained at $0.33 \mathrm{Atm}$. high purity helium. An additional $0.17 \mathrm{Atm}$. was used to eject the melt from the quartz tube.

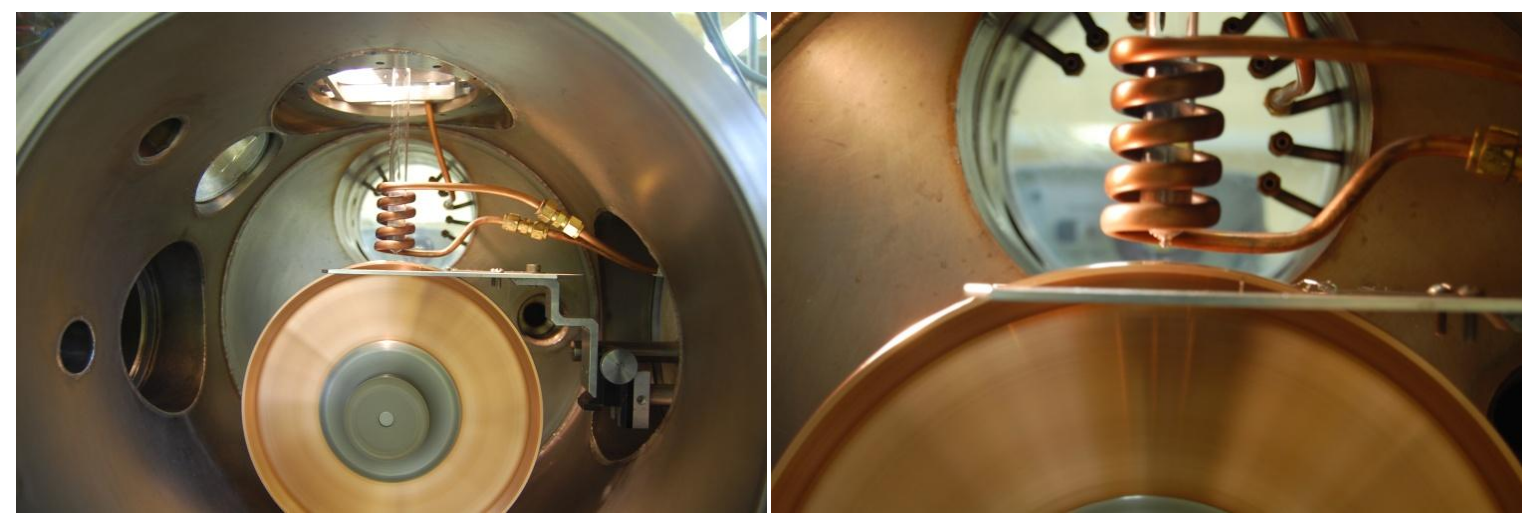

Figure 21 - Melt-spinning setup

A single layer of the mostly-amorphous precursor ribbons was placed between two pieces of boron nitride-coated tantalum foil. This assembly was placed in a $1.27 \mathrm{~cm}$ diameter graphite die. The die was then placed in an inductively heated vacuum hot press. A vacuum of roughly $10^{-6}$ Torr was achieved prior to heat 
treatment. The die was heated at $30^{\circ} \mathrm{C} /$ minute until the desired temperature was reached. If uniaxial pressure was desired, the pressure was applied prior to heating.

\section{2 - Results}

Mostly-amorphous $\mathrm{Nd}_{0.8} \mathrm{Y}_{0.8} \mathrm{Dy}_{0.4} \mathrm{Fe}_{14} \mathrm{~B}+3 \mathrm{wt} \% \mathrm{TiC}$ was formed through arc melting and melt-spinning as described above in section 4.1. Differential scanning calorimetry was completed on the ribbons. Figure 22 shows the resulting thermal profile. An exothermic crystallization event with an onset temperature of $624^{\circ} \mathrm{C}$ can be seen in the trace. This indicates that a significant fraction of amorphous material is present in the ribbon.

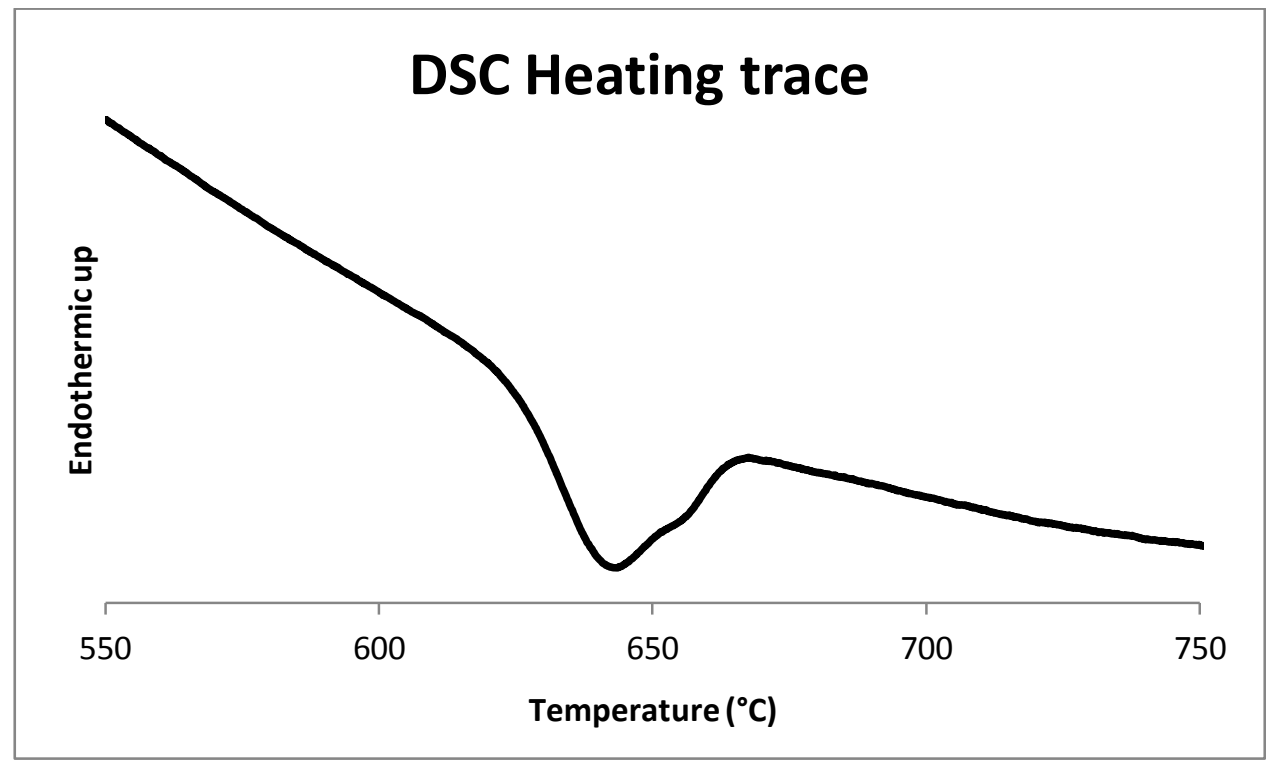

Figure 22 - DSC heating trace of as-spun $\mathrm{Nd}_{0.8} \mathrm{Y}_{0.8} \mathrm{Dy}_{0.4} \mathrm{Fe}_{14} \mathrm{~B}+3 \% \mathrm{TiC}$

TEM was also done on the as-spun ribbons. Figure 23 shows a bright field TEM image of the as-spun ribbon. The structure observed is mostly disordered, with a single crystallite of 2-14-1 observed (indicated by the arrow in the figure). The selected area diffraction pattern shows broad, diffuse rings, indicating a mostly amorphous structure.

X-ray diffraction was also completed on the as-spun ribbons using Mo k- $\alpha$ radiation. The instrument used was a Stoe IPDS II diffractometer with a Debye- 
Scherrer geometry and 2-dimensional detector. The resulting diffraction pattern shows diffuse rings with a small number of higher intensity diffracted spots indicating crystallites. This confirms a structure that is largely amorphous, with a small number of crystalline regions. Figure 24 shows the two dimensional diffraction pattern observed (left) and the one dimensional integrated trace (right).
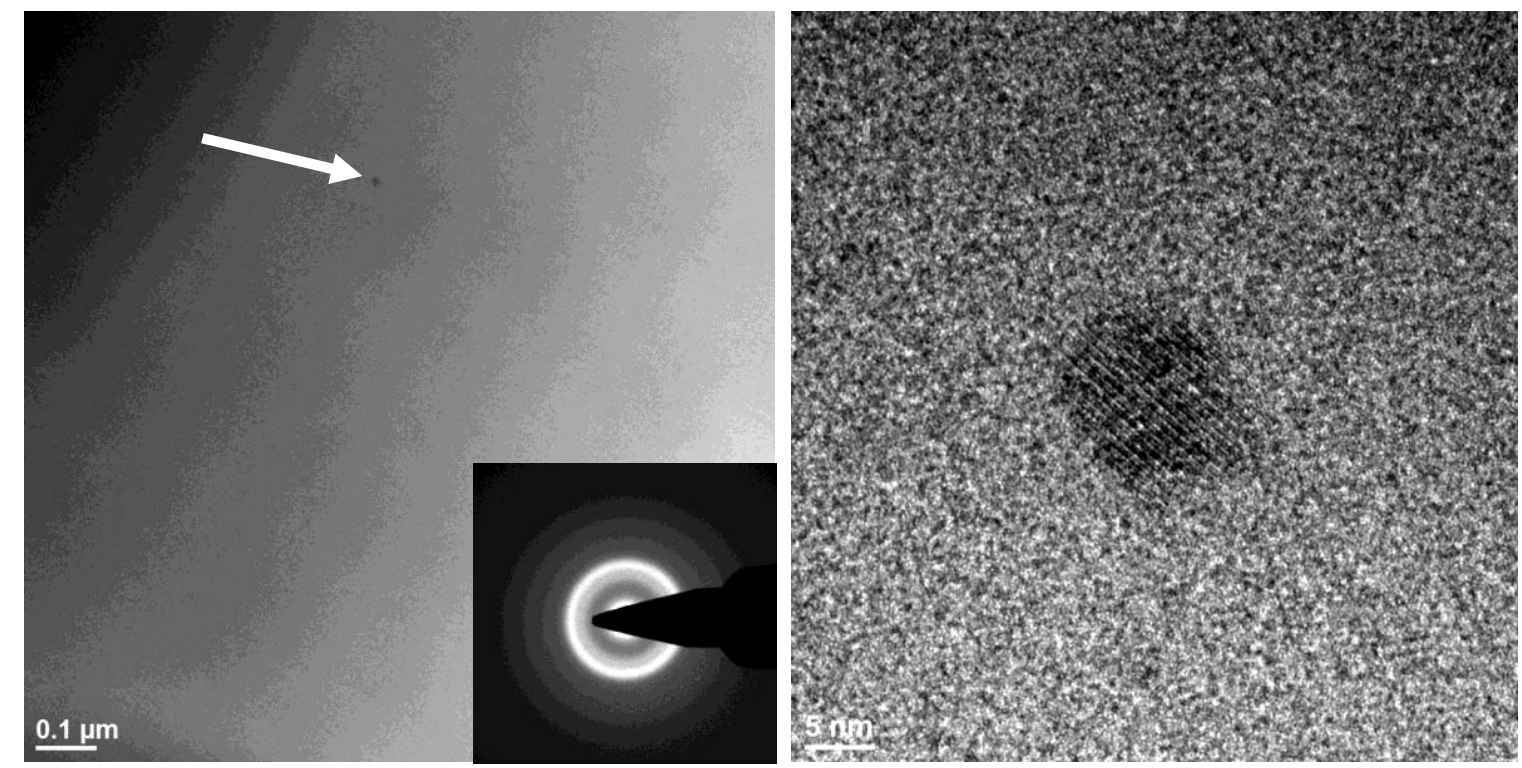

Figure 23 - Bright field TEM image of as-spun ribbon and corresponding selected area diffraction pattern (SADP) (left). The arrow points to the only crystalline material observed. On the right is a close up image of the crystallite.

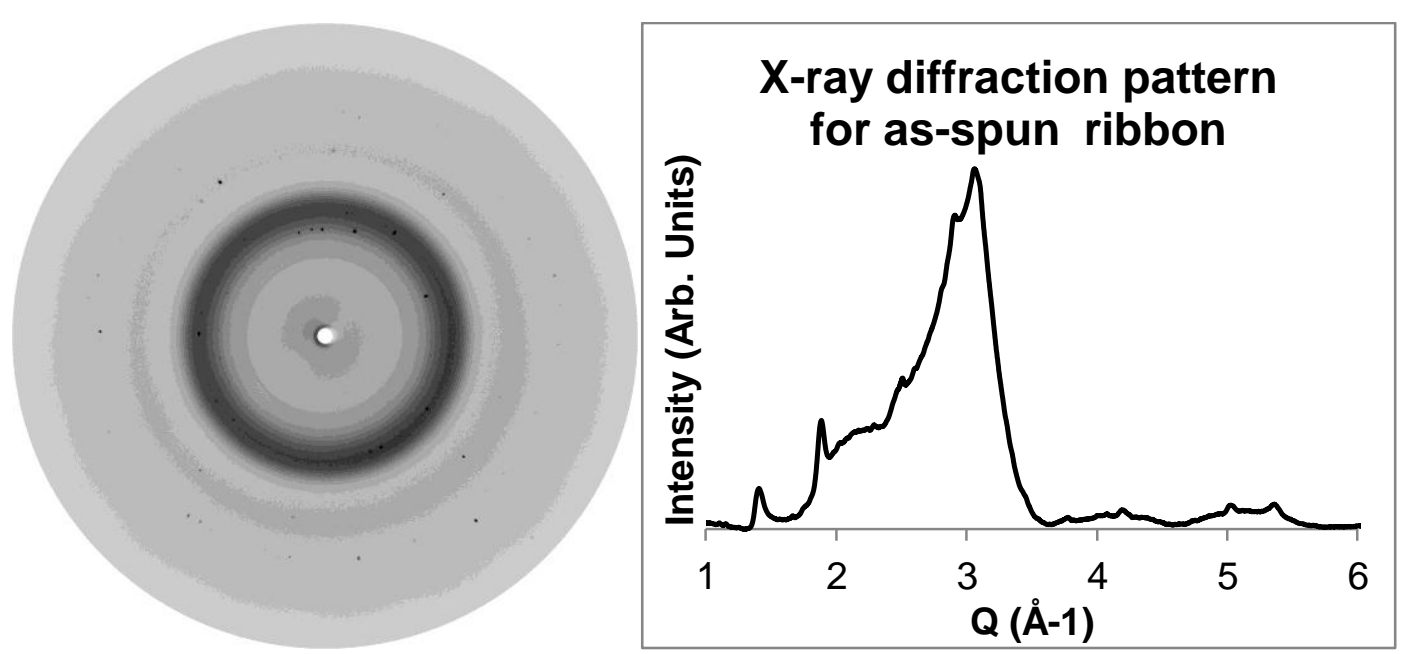

Figure 24 - X-ray diffraction pattern of as-spun $\mathrm{Nd}_{0.8} \mathrm{Y}_{0.8} \mathrm{Dy}_{0.4} \mathrm{Fe}_{14} \mathrm{~B}+3 \mathrm{wt} \% \mathrm{TiC}$ 
The as-spun ribbon was then vacuum hot pressed at $580^{\circ} \mathrm{C}$ and held for one hour as described above. For the control case, no uniaxial pressure was applied during pressing. In another case, a uniaxial pressure of $120 \mathrm{MPa}$ was applied prior to heating and held throughout. The resulting ribbons were mounted on a copper slot grid and ion milled for TEM preparation. In the control case, TEM images show a microstructure with grain size on the order of $50-70 \mathrm{~nm}$. The grain boundaries are decorated with $\mathrm{Ti}$ - and $\mathrm{C}$-rich areas. This microstructure is typical of isotropic meltspun alloys. The structure observed in the sample where pressure was applied, however, showed an unusual defect structure. The mottled appearance and poorly defined grain boundaries indicate a high defect density.

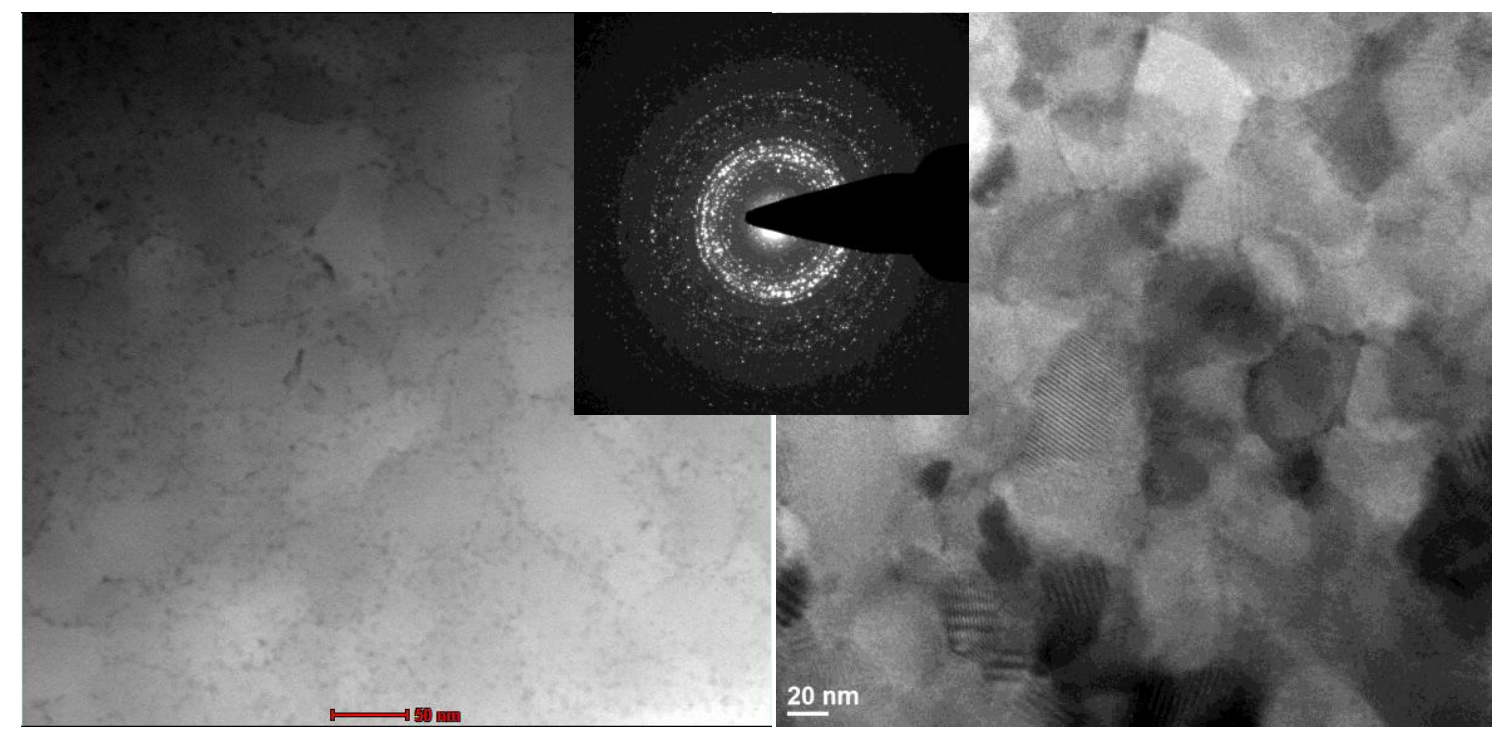

Figure 25 - High angle annular dark field (HAADF) scanning transmission electron microscopy (STEM) (left) and bright field (right) TEM images of sample annealed under no uniaxial pressure and the corresponding SADP.

X-ray diffraction was also done on the samples. The diffraction patterns are shown below in Figure 27. It can easily be seen that significant texturing is caused by the application of pressure. This is shown by the large number of suppressed peaks. 


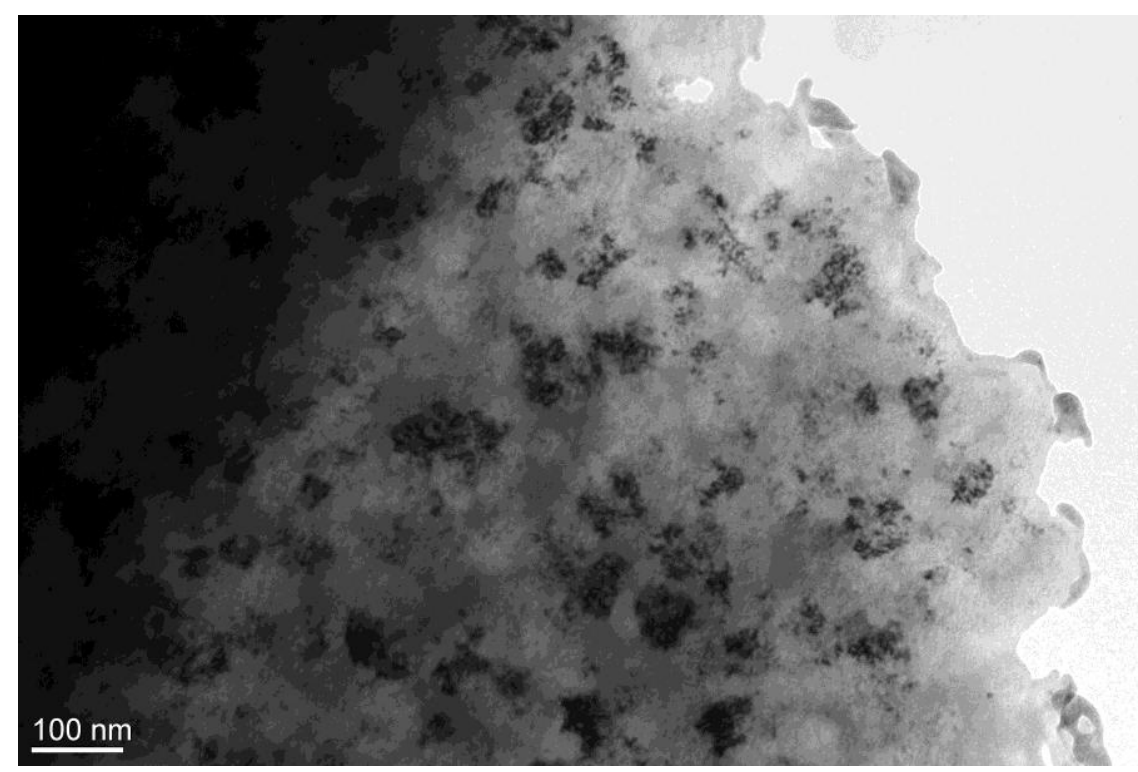

Figure 26 - Bright field TEM image of sample annealed under $120 \mathrm{MPa}$ uniaxial pressure.

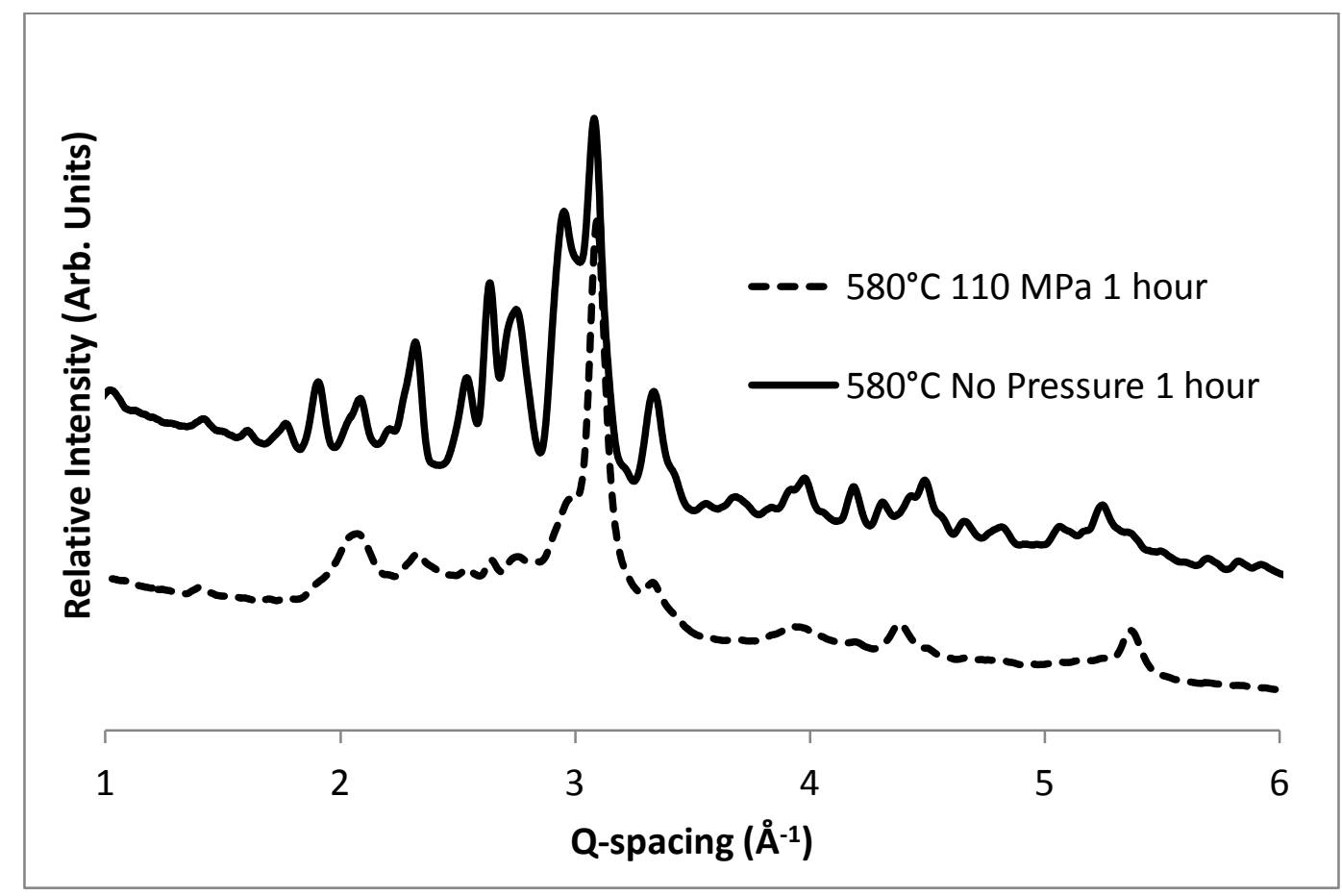

Figure 27 - X-ray diffraction pattern of heat treated ribbons with and without applied uniaxial pressure 
Hysteresis curves for the two samples were also measured using a SQUID magnetometer. Curves were measured both parallel and perpendicular to the pressing direction. Figure 28 shows the obtained curve for the sample treated with no applied pressure. Figure 29 shows the curves for the sample treated with $120 \mathrm{MPa}$ applied pressure. No units are given for the vertical axis since the ribbon fragments had residual boron nitride attached to them and, therefore, magnetization could not accurately be reported due to uncertainty of the sample mass. Units along the horizontal axis are kOe.

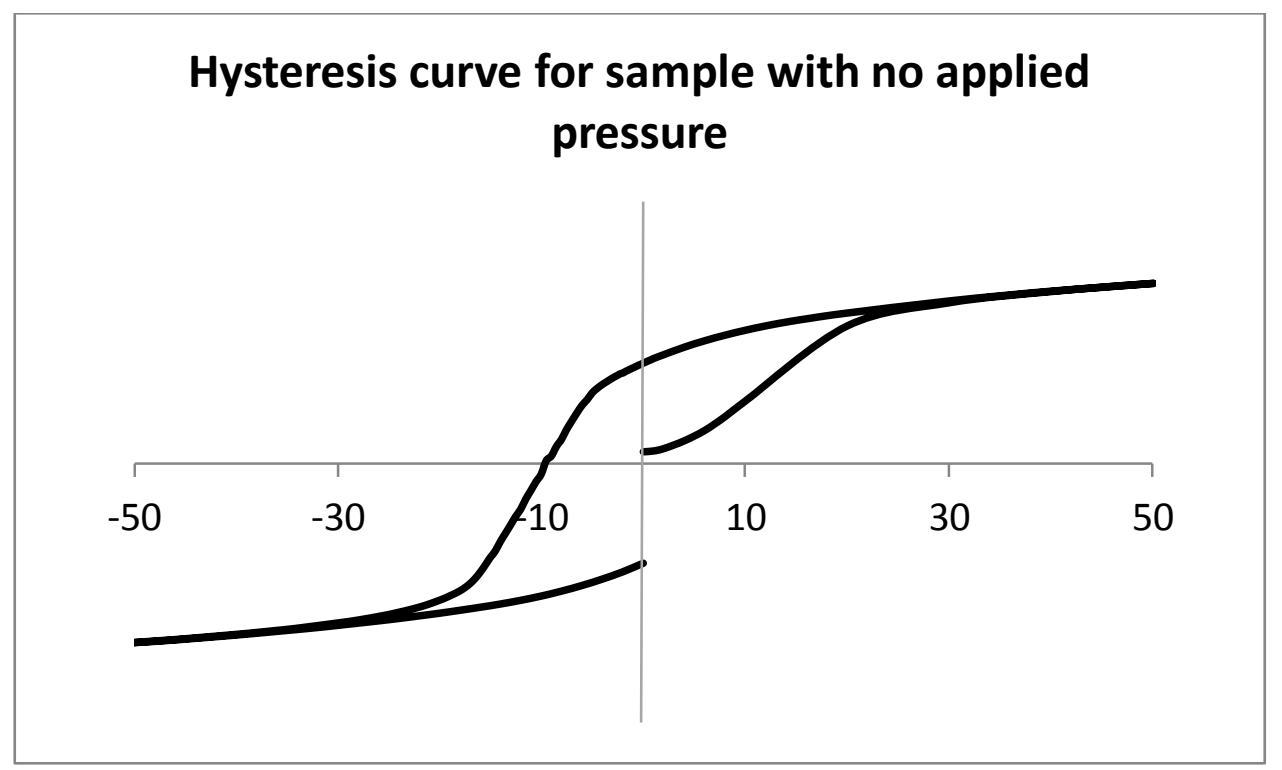

Figure 28 - Hysteresis curve for sample heat treated with no applied pressure. 


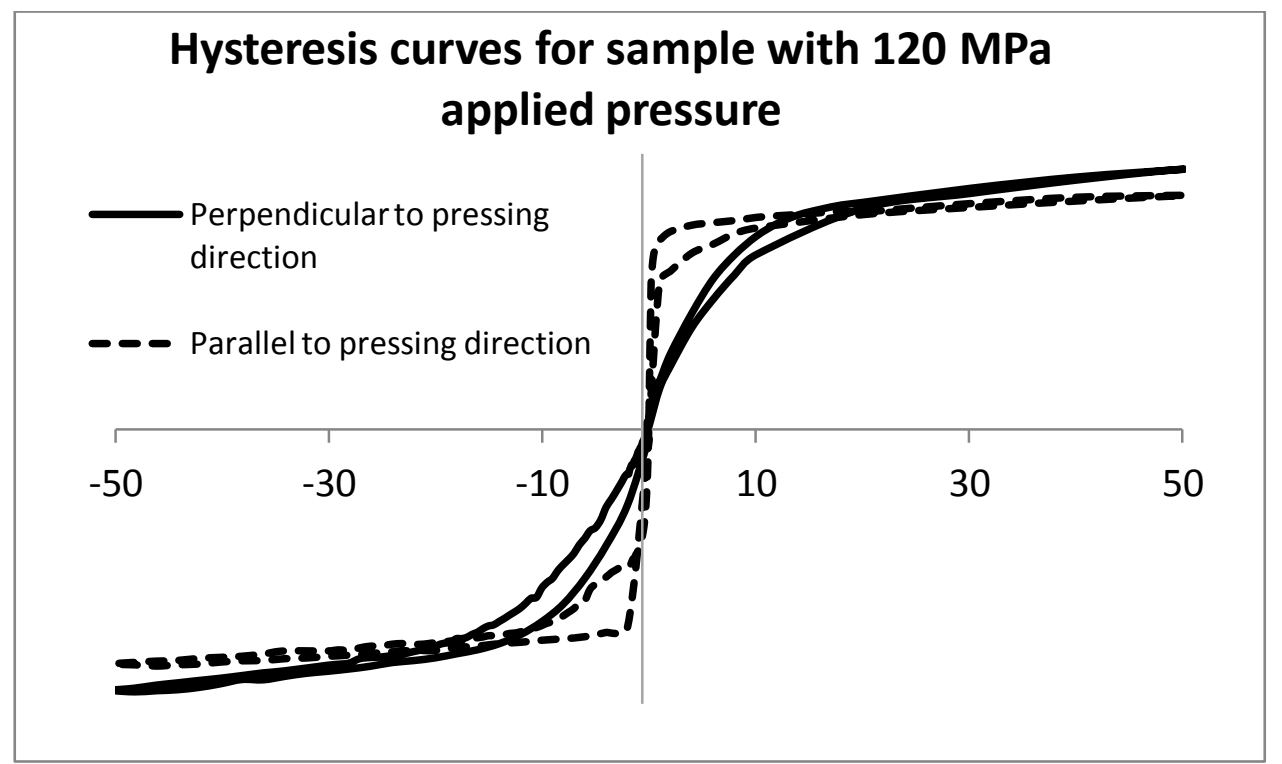

Figure 29 - Hysteresis curves for the sample annealed under uniaxial pressure taken in the directions parallel and perpendicular to the pressing direction.

\section{3 - Discussion}

Creation of suitable precursor amorphous ribbon was successfully created through the alloy selection and processing parameters. DSC, TEM, and XRD measurements all confirmed that a largely amorphous structure was present. In the TEM and XRD measurements, it is apparent that a portion of the material contains a number of crystalline regions. While the material formed was not fully amorphous, it should not be an issue. Since the observed crystallites were very small, it is unlikely that excessive growth of these nuclei should be a major problem. It may even be helpful to have some heterogeneous crystallization sites in the bulk of the material in order to reduce the temperature needed to crystallize the structure.

The application of uniaxial pressure during crystallization was seen to have a strong effect on the observed microstructure and texture in these mixed rare earth-iron-boron alloys. The TEM images of the material crystallized without applied pressure displayed microstructures very comparable to isotropic alloys which have been overquenched and annealed. It is likely that there is a relatively high degree of crystal perfection within the individual grains, as lattice fringes can be easily seen in 
the bright field image. Grain boundaries are also easily discerned. The HAADF image shows that the titanium and carbon segregate to the grain boundaries. The hysteresis curve for the sample with no applied pressure very closely resembles what would be expected of rapidly solidified isotropic particulate like what is predicted by Stoner and Wohlfarth [23] in Figure 10. It can be concluded that the control case results in a typical isotropic RE-Fe-B magnet.

In contrast, the sample that had pressure applied during crystallization displayed properties atypical of isotropic magnet particulate. Most notably, the microstructure is drastically different. The mottled appearance of the grains indicates a high defect density. In addition, lattice fringes are not easily seen, further suggesting imperfection within the crystal lattice. Grain boundaries are not well defined in this structure and the TiC cannot be easily distinguished from the RE-Fe$B$ matrix. This defect structure is not seen in die upset material or cold pressed material. In fact, no slip systems are activated within the 2-14-1 crystal structure until temperatures upwards of $900^{\circ} \mathrm{C}$ are reached. This was determined by Kuhrt et al. in their study of high temperature compression of $\mathrm{Nd}_{2} \mathrm{Fe}_{14} \mathrm{~B}$ single crystals [75]. This indicates that the defect structure is likely locked into place during crystallization. In cold pressing of the material, fracture would occur prior to defect formation. In the die upset process, grain boundary diffusion takes place, not deformation of the crystals themselves. The defects observed are likely a function of the restricted geometry and a strong driving force to form crystalline material. In addition to formation of the defects, a preferred c-axis texture is also created.

One other likely result of the defect structure is loss of coercivity. While strain within the lattice can effectively pin domain walls through increasing the magnetoelastic energy of the system, an increase in coercivity is not observed. Two different explanations can be used to describe the result: increased coupling of neighboring grains or too high of a defect density to pin domain walls. In section 3.7, a discussion of various additives were discussed. One class of these additions is intended to segregate to the grain boundaries. The effect is that the grain boundaries are widened such that they are on the order of the domain wall 
thickness. This helps decouple grains, so a domain cannot easily pass from one grain to the next. If the defect structure were to effectively blur the grain boundaries, it is possible that the grains may couple more easily and the boundaries may no longer act as strong domain pinning sites. The second possibility is that the defect density is simply so high that a domain wall would not be able to distinguish one defect from the next. As a result, the overall potential the domain wall sees would have little variation and no defects would pin the domain wall. It is possible that the material could be annealed to reduce strain within the grains. However, texture might be reduced upon recrystallization.

One of the primary objectives of this work is to minimize processing costs. The controlled devitrification approach requires several processing steps. Meltspinning to create amorphous precursor, heating under applied pressure, and a likely annealing step. The process is somewhat similar to the die upset processing route. It is important to note that the die upset method has not been deemed a commercially viable processing route. It is likely that this route would be subject to many of the same hurdles. Additionally, scaling this process to a commercial scale may be difficult, if not impossible.

\section{4 - Conclusions}

- Creation of nanocrystalline aligned structures can be achieved through devitrification of amorphous material under applied uniaxial stress.

- Melt-spinning at high wheel speeds, in combination with the addition of TiC, can produce suitable amorphous precursor material for controlled devitrification.

- Processing the material in the same manner, but without applied stress, results in a nanocrystalline isotropic ribbon.

- A high defect density is created within the sample when crystallized under uniaxial pressure, resulting in degraded coercivity. 


\title{
Chapter 5 - Effects of wheel surface conditions on phase assemblage and glass formation in melt-spun TiC-modified $\mathrm{Nd}_{2} \mathrm{Fe}_{14} \mathrm{~B}$
}

\author{
A paper to be submitted to Metallurgical and Materials Transactions $B$ \\ N.T. Oster, D.T. Cavanaugh, K.W. Dennis, R.W. McCallum, M.J. Kramer, I.E. \\ Anderson
}

\begin{abstract}
Melt-spinning is a commonly used rapid solidification method for production of nanocrystalline Nd-Fe-B magnet alloys. The final structure of the ribbons is dictated, in part, by the various processing parameters. Wheel speed and melt superheat are two of the primary factors affecting the final ribbon. In this work, two additional parameters are investigated-wheel temperature (WT) and surface finish. Ribbons of composition Fe-11Nd-6B-3Ti-3C (at\%) were formed by melt spinning on a copper wheel that was maintained at 25,100 , or $200^{\circ} \mathrm{C}$. The wheel was polished with either $320,400,600$, or $800 \mathrm{SiC}$ abrasive paper. Tangential wheel speed was varied from $10-30 \mathrm{~m} / \mathrm{s}$. The effects of the wheel temperature and surface finish on phase assemblage and glass formation of the alloy were investigated through differential scanning calorimetry, $x$-ray diffraction, and SQUID magnetometry. Surface features were characterized by scanning electron microscopy, optical microscopy, and optical profilometry. Melt-spinning onto a copper wheel at $200^{\circ} \mathrm{C} W T$ resulted in greater formation of amorphous phase than the lower WT values. Finer polishes on the wheel surface resulted in decreased amorphous material formation. The authors propose that the change in ability to form amorphous material is caused by modification of the interface between melt and wheel.
\end{abstract}

\section{Introduction}

Controlling the microstructure and phase assemblage of Nd-Fe-B alloys is of critical importance in obtaining desirable magnetic properties for high performance 
permanent magnets (PMs). One processing route that has been commonly used is melt-spinning $[11,76,77]$. This processing route allows for rapid solidification of the melt and fine grain structures. Overall cooling rates of $\sim 10^{4}-10^{6} \mathrm{~K} / \mathrm{s}$ have been reported[78]. For Nd-Fe-B alloys, near-stoichiometric alloys are rapidly solidified to an "over-quenched" state[59], where nanocrystalline grains and amorphous material are present, followed by a flash anneal to crystallize the remaining amorphous fraction. The resulting microstructure consists of equiaxed, nanocrystalline grains $(\sim 50 \mathrm{~nm})$. Control of this microstructure is of critical importance to maintain adequate magnetic properties.

Several melt-spinning processing parameters and alloy considerations have been investigated regarding control of the as-spun microstructure and phase assemblage. Several works have observed the effects of tangential wheel speed during melt-spinning on the final microstructure and magnetic properties [59, 79]. Low wheel speeds $(5-15 \mathrm{~m} / \mathrm{s})$ result in a fine, equiaxed structure at the surface, with a coarser columnar structure extending out of this region. The relatively large grain structure results in low coercivity. High wheel speeds $(>20 \mathrm{~m} / \mathrm{s})$ result in fine $(<70$ $\mathrm{nm}$ ) grains and amorphous material. Branagan and McCallum, Kramer et al., and Filip et al. observed the effects of additions of Ti, C, and TiC[58, 60, 61]. They determined that these additions ( $\mathrm{TiC}$, in particular) increased the fraction of amorphous material observed at high wheel speeds, and reduced grain growth at lower wheel speeds.

Several studies have also documented the effects of chamber atmosphere on the properties of the ribbon $[80,81]$. It was found that optimal quench properties were achieved when $\sim 1 / 3 \mathrm{~atm}$. of He was used. When higher pressures of the $\mathrm{He}$ chamber atmosphere were used, excessive amounts of gas were entrapped between the melt and the wheel, reducing the overall heat transfer rate. Several other studies have related the properties of the ribbon to the interfacial conditions $[82,83]$. In this work, the interfacial conditions are observed through variation of the wheel surface finish. 
Some work has also been devoted to observing the effects of WT on cooling rate and the amorphous content in resulting melt spun ribbons. Wang and Matthys [84] calculated the effects of wheel material and melt superheat on the thermal profiles in both the wheel and melt during planar flow melt-spinning. They determined that solidification front velocity (and presumably amorphous content) increases with wheel material of increasing thermal conductivity. Additionally, large degrees of localized heating under the melt pool were predicted, enough that the wheel temperature (WT) would increase with successive wheel rotations for an uncooled wheel. Most experimental work concerning WT focuses on actively cooling the wheel, but one of the co-authors (RWM) had performed a preliminary analysis that suggested that modest heating of the wheel may also increase amorphous content. Thus, the work presented here documents the effects of intentionally heating the wheel on phase selection and amorphous content.

\section{Experimental Procedure}

Ingots of $\mathrm{Fe}-11 \mathrm{Nd}-6 \mathrm{~B}-3 \mathrm{Ti}-3 \mathrm{C}$ (at\%) were arc melted from the constituent elements using a non-consumable tungsten electrode on a water-cooled copper hearth. TiC was added to increase the ability of the alloy to form amorphous material. The ingots were then free jet melt-spun on a rotating copper wheel. Chamber atmosphere was maintained at 250 Torr high purity He. To prepare the atmosphere, the chamber was evacuated to approximately $50 \times 10^{-3}$ Torr, backfilled, then evacuated to less than $10^{-3}$ Torr prior to backfilling with He.

The wheel used was approximately $0.4 \mathrm{~m}$ in diameter with a mass of roughly $100 \mathrm{~kg}$. This melt-spinning apparatus was specially designed and built in the Ames Laboratory to have the ability to inductively heat the wheel. For these experiments, the surface finish of the wheel was varied by polishing the wheel using varying grit sizes of $\mathrm{SiC}$ grinding papers. $320,400,600$, and 800 grit papers were used (average abrasive particle diameters are $36.0 \mu \mathrm{m}, 26.3 \mu \mathrm{m}, 16.0 \mu \mathrm{m}$, and $12.2 \mu \mathrm{m}$, respectively). To prep the wheel surface, the wheel was polished using fresh sheets of the various grit sizes for 3 minutes each, starting with the coarsest grit paper (320) and proceding until the finest desired grit size was used. The 
surface was then cleaned using ethanol. The ingots were inductively heated to $1400^{\circ} \mathrm{C}$ and ejected from a quartz crucible with a $0.8 \mathrm{~mm}$ circular orifice using an ejection pressure of 120 Torr of high purity He.

In one group of experiments, the wheel was polished to 600 grit, tangential wheel speed was varied from $10 \mathrm{~m} / \mathrm{s}$ to $30 \mathrm{~m} / \mathrm{s}$, and wheel temperature was set to either $25^{\circ} \mathrm{C}$ or $200^{\circ} \mathrm{C}$. In a second group of experiments, tangential wheel speed was maintained at $15 \mathrm{~m} / \mathrm{s}$, wheel surface was varied (polished to either $320,400,600$, or 800 grit), and wheel temperature was varied $\left(25^{\circ} \mathrm{C}, 100^{\circ} \mathrm{C}\right.$, or $\left.200^{\circ} \mathrm{C}\right)$.

\section{Experimental Results}

\section{III. a. Varied Wheel Speed and Wheel Temperature}

\section{Differential Scanning Calorimetry}

Differential scanning calorimetry (DSC) was done on each melt-spun alloy to determine the relative fraction of amorphous material (increased crystallization peak area corresponds to increased amorphous fraction) with a Netzsch DSC 404C calorimeter. Approximately $45 \pm 5 \mathrm{mg}$ of ribbon pieces were used from each run to normalize the results. To ensure good sampling of the ribbon, 3-5 separate scans for each batch of ribbon were tested. The ribbons were placed in an $\mathrm{Al}_{2} \mathrm{O}_{3}$ crucible and heated at $20^{\circ} \mathrm{C} / \mathrm{min}$ to $750^{\circ} \mathrm{C}$ in a flowing high purity He atmosphere. A sample trace can be seen below in Figure 30. Since a large amount of amorphous material can be obtained, many of the ribbon sets displayed an exothermic crystallization peak, observed at approximately $600^{\circ} \mathrm{C}$. The area of each peak was measured using the Netzsch software provided with the calorimeter. Figure 31 shows the crystallization peak area as a function of tangential wheel speed for the room temperature and $200^{\circ} \mathrm{C}$ wheel. The error bars represent a 95\% confidence interval. Larger peak areas indicate greater fractions of amorphous material. The amount of amorphous material present in the samples melt-spun on a room temperature wheel showed a nearly linear increase in amorphous content with tangential wheel speed. The observation that amorphous content in TiC-containing alloys increases with increasing wheel speed for a typical ambient WT is consistent with previous studies $[4,58,60]$. In all 
cases displaying any amorphous content, it is interesting that the elevated WT resulted in a greater amorphous content than the room temperature wheel for a given wheel speed.

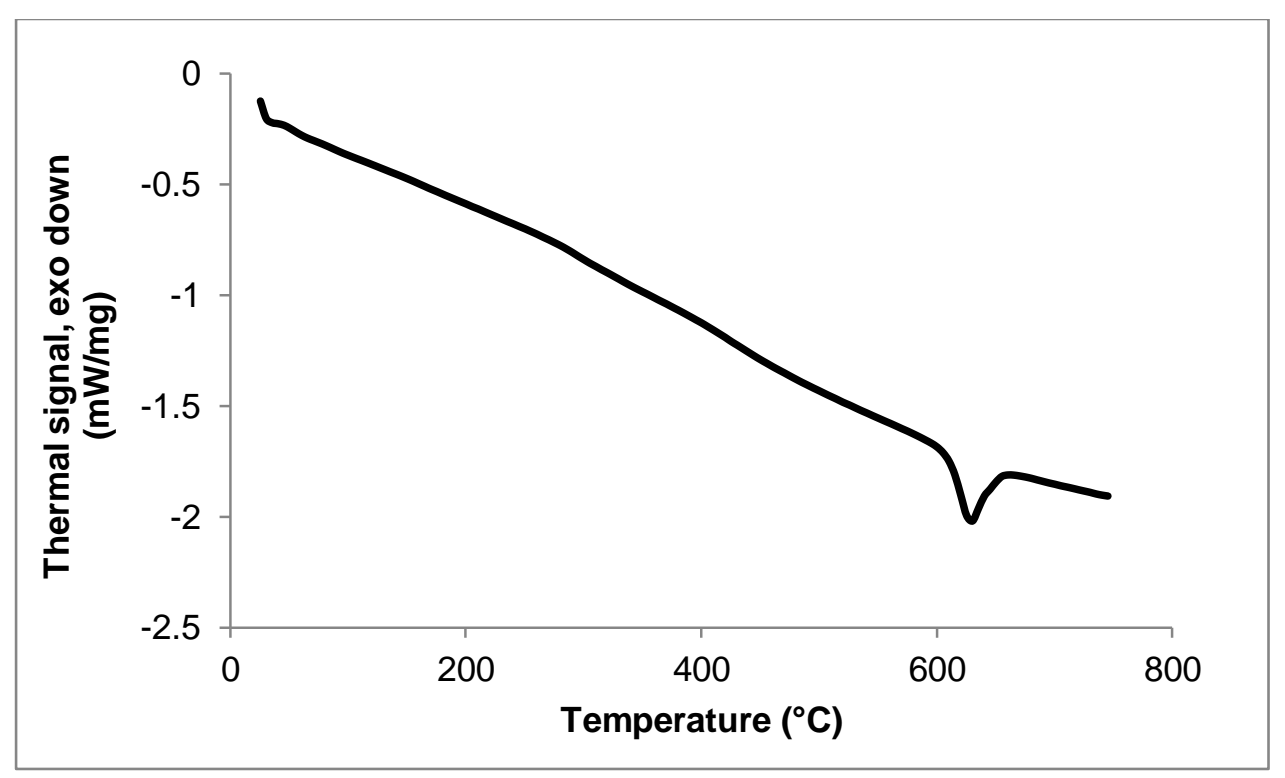

Figure 30 - DSC trace for $\mathrm{Nd}_{4} \mathrm{Fe}_{28} \mathrm{~B}_{2} \mathrm{TiC}$ melt-spun at $20 \mathrm{~m} / \mathrm{s}$ tangential wheel speed onto a room temperature $\mathrm{Cu}$ wheel 


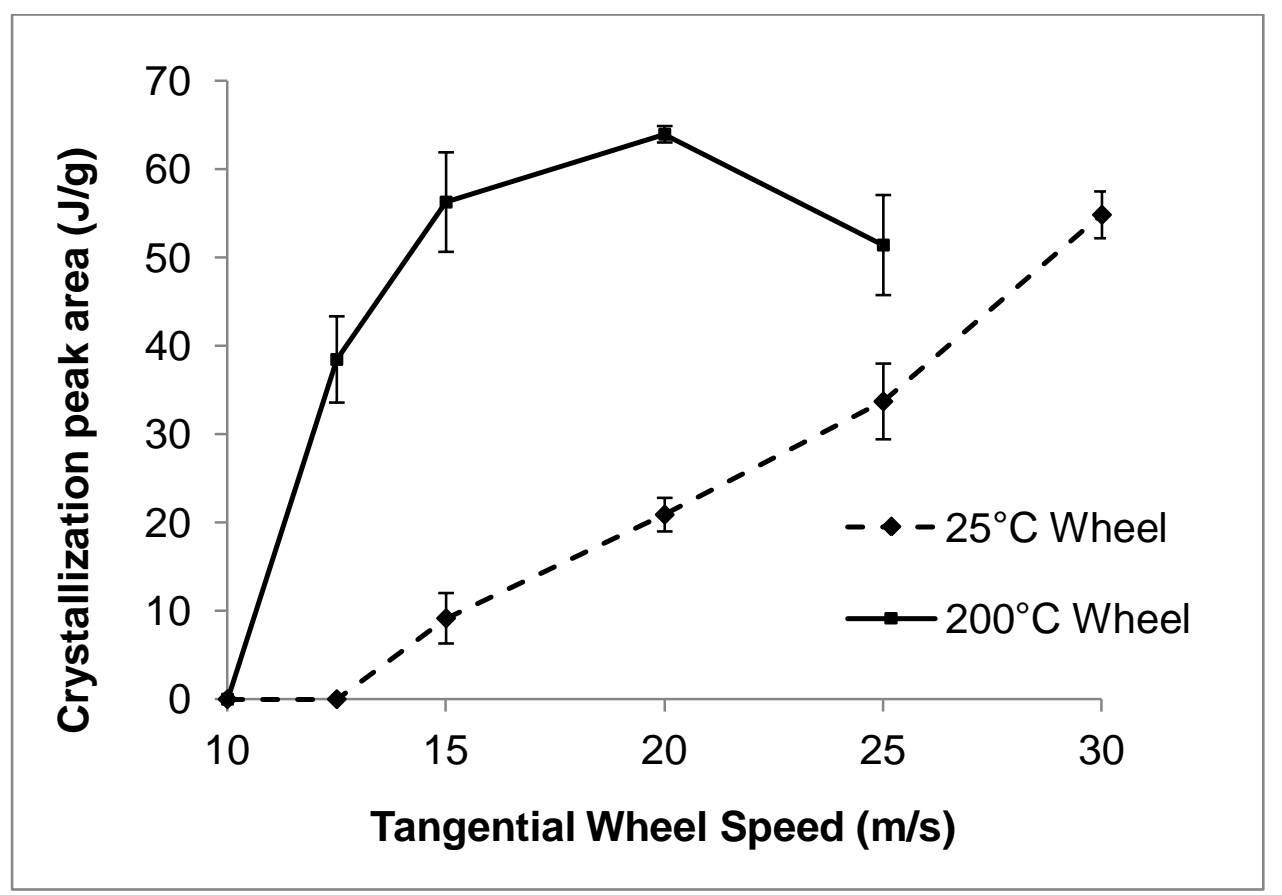

Figure 31 - Exothermic crystallization peak area as a function of tangential wheel speed for two different wheel temperatures (as determined by DSC). Error bars represent a $95 \%$ confidence interval.

\section{Ribbon Thickness}

Ribbon thickness was measured using a rounded-tip micrometer with accuracy of $1 \mu \mathrm{m}$. For each set of ribbons, approximately 25 measurements were taken from different ribbon pieces. Figure 32 shows the ribbon thickness as a function of wheel speed for each WT. The error bars indicate a 95\% confidence interval. It can be seen that ribbon thickness decreases with increasing wheel speed. Thus, wheel surface temperature has no statistically significant effect on the thickness of the obtained ribbons. 


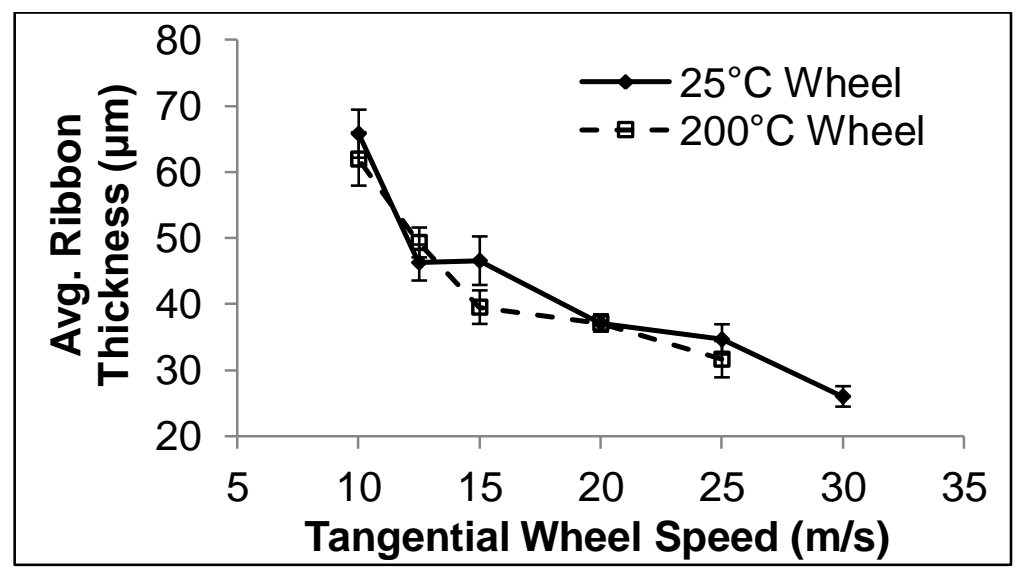

Figure 32 - Average ribbon thickness. Error bars represent a 95\% confidence interval.

\section{$X$-ray Diffraction}

To determine phase assemblage, $x$-ray diffraction was completed on each sample. Approximately 10 ribbon pieces were adhered to a glass slide using doublesided tape with either the wheel side or the free side facing up. Diffraction patterns were taken using a Panalytical x'Pert Pro diffractometer with $\mathrm{Cu}$ K- $\alpha$ radiation.

Figure 33 illustrates the differences in the patterns seen between the wheel and free sides of the ribbons.

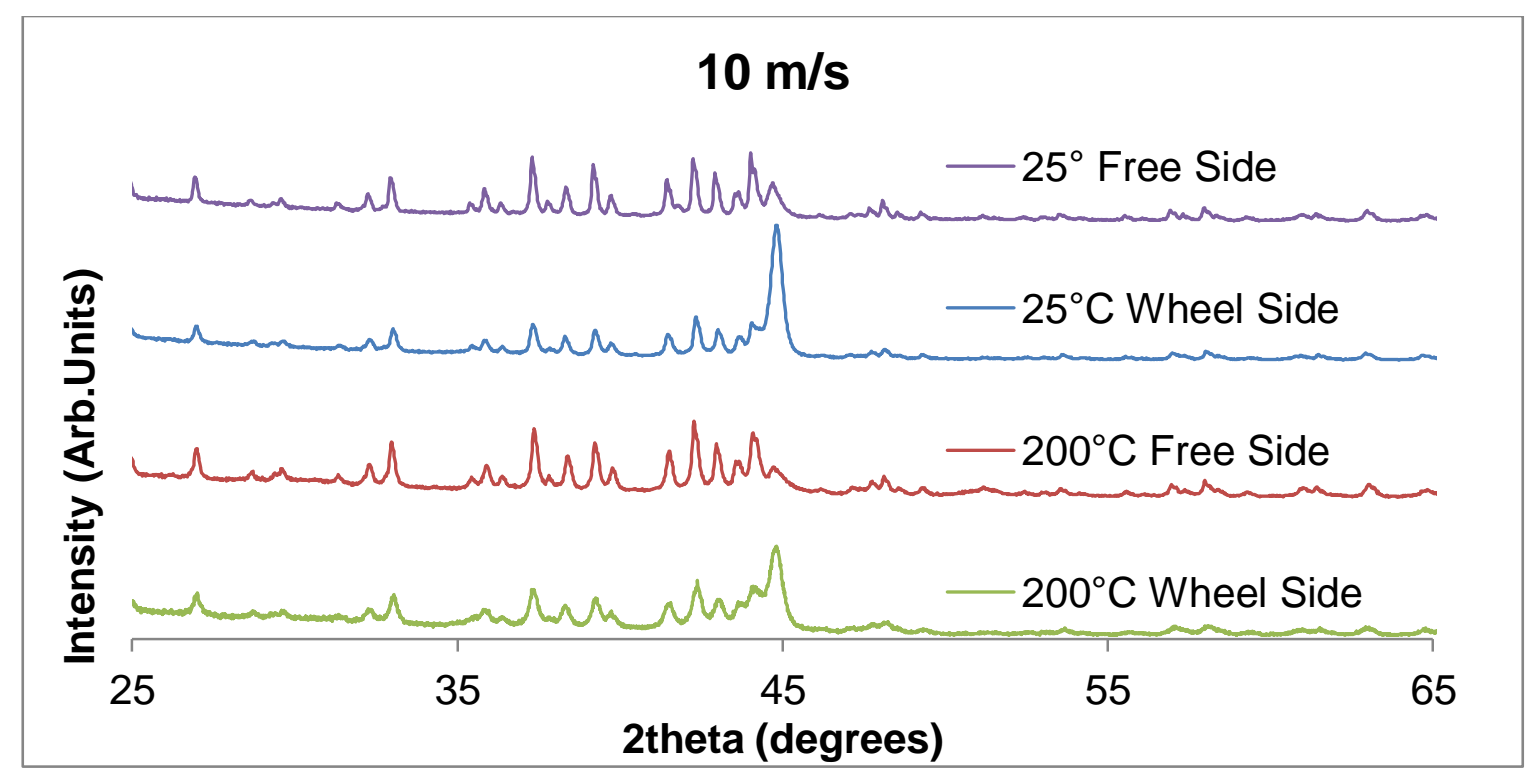

Figure 33 - XRD Traces for ribbons melt-spun at $10 \mathrm{~m} / \mathrm{s}$ tangential wheel velocity. 

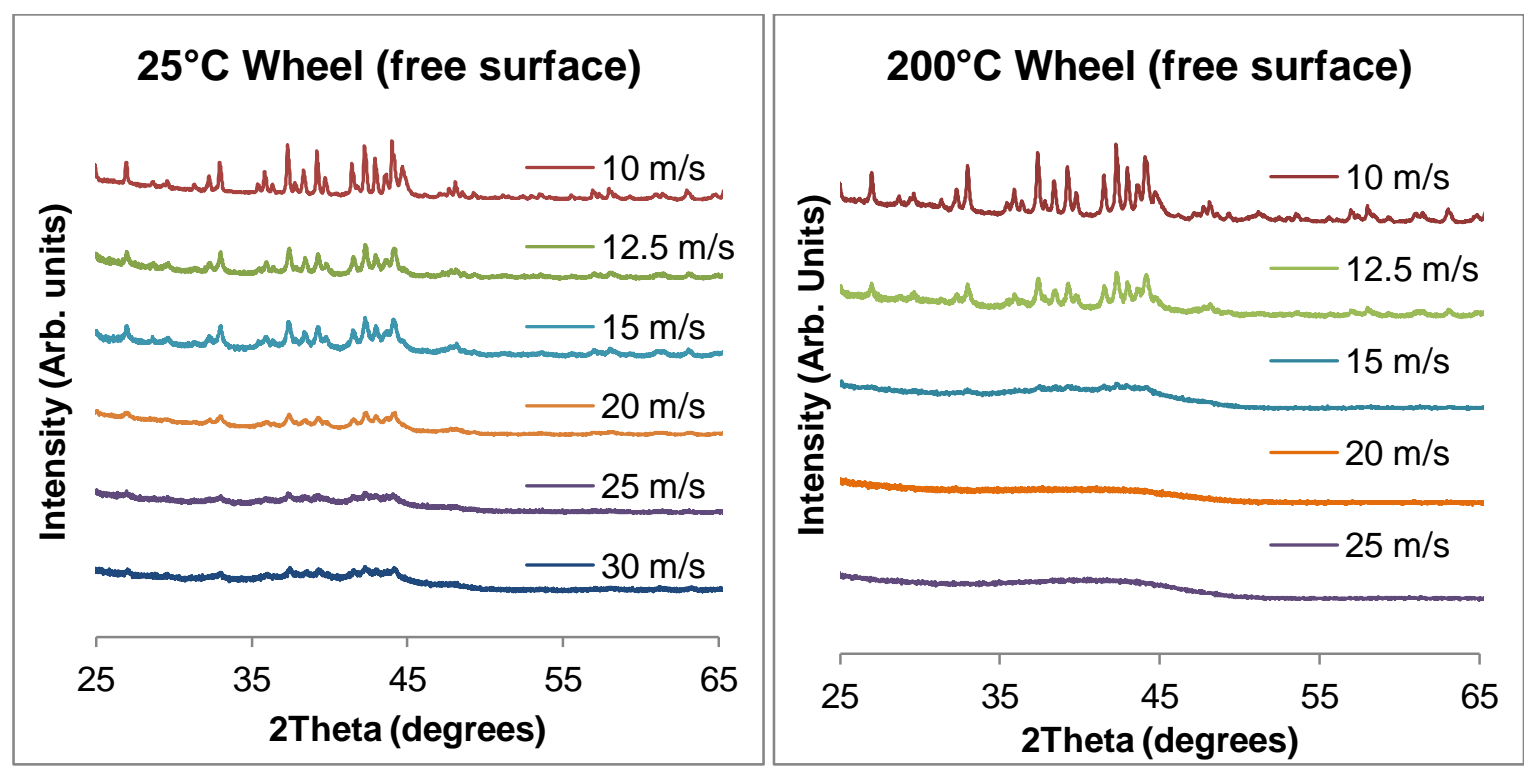

Figure $34-X R D$ traces of free surfaces of ribbons melt-spun using a WT of a) $25^{\circ} \mathrm{C}$ or b) $200^{\circ} \mathrm{C}$.

In the samples melt-spun on a $25^{\circ} \mathrm{C}$ wheel, none of the ribbon batches resulted in a fully amorphous structure (see Figure $34 \mathrm{a}$ ). When a $200^{\circ} \mathrm{C}$ wheel was used, the traces from ribbons melt-spun at 20 and $25 \mathrm{~m} / \mathrm{s}$ indicated a fully amorphous structure (see Figure 34b). In all crystalline material, the peaks correspond well to the $\mathrm{Nd}_{2} \mathrm{Fe}_{14} \mathrm{~B}$ intermetallic structure. On the wheel surface scans containing significant crystalline material, the peaks at $44.8^{\circ} 2 \theta$ are enhanced. This is (presumably) caused by primary Fe dendrites (either still present in the microstructure, or consumed during formation of the $\mathrm{Nd}_{2} \mathrm{Fe}_{14} \mathrm{~B}$ structure). Kramer et al.[59] proposed a texturing mechanism where the 2-14-1 crystals template on the Fe dendrites (which form first and preferentially grow along their $<100>$ directions). If the iron were not present, the peak would not be enhanced. This enhancement allows one to assume that $\mathrm{Fe}$ is one of the primary solidifying phases in this system in all crystalline samples. 


\section{SQUID Magnetometer}

Saturation magnetization was measured using a Quantum Design MPMS SQUID magnetometer. $35 \pm 15 \mathrm{mg}$ of ribbon pieces from each melt-spinning trial were exposed to a $50 \mathrm{kOe}$ applied field. Three different samplings were taken from each trial. The resulting magnetizations for each wheel temperature are shown in Figure 35 as a function of wheel speed. This data is consistent with the expected trend in saturation magnetization that is inversely related to crystallization energy, resulting from the difference in Fe bonding state between amorphous and crystalline materials.

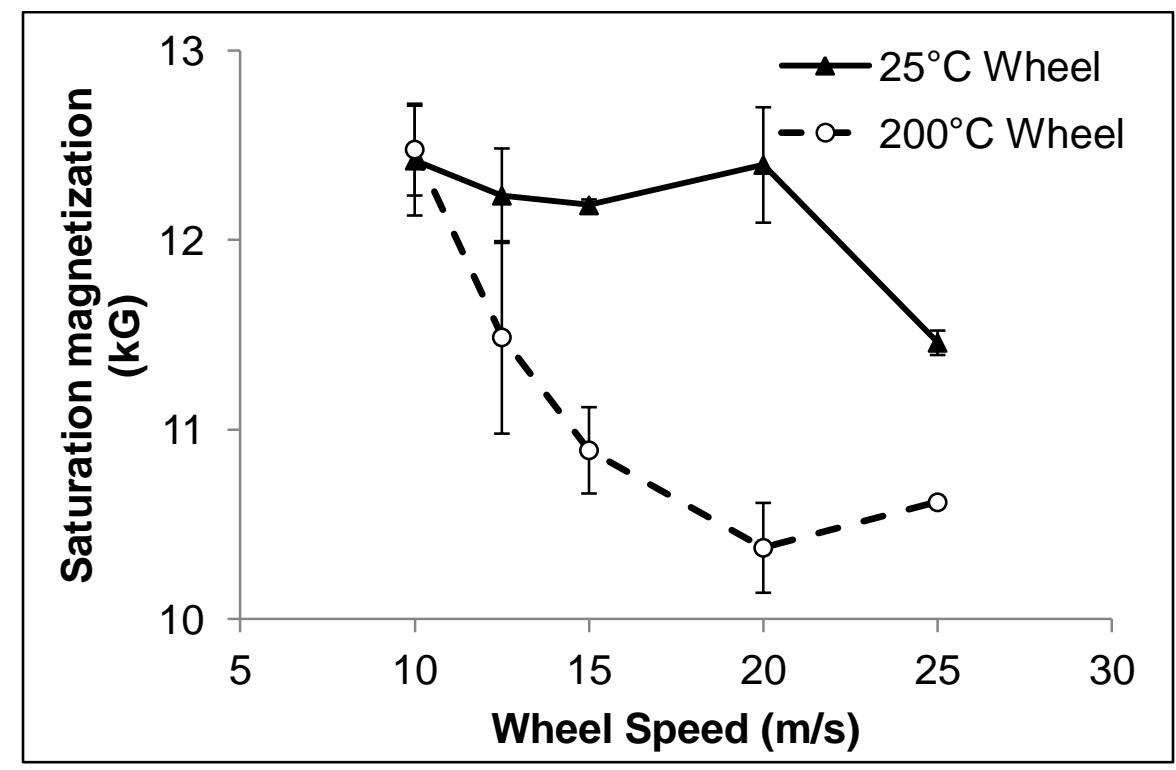

Figure 35 - Saturation magnetization as a function of wheel speed. Error bars represent a $95 \%$ confidence interval.

\section{III. b. Varying Surface Finish and Wheel Temperature}

\section{Differential Scanning Calorimetry}

DSC was performed on each of the samples where surface finish and temperature were both varied as described above. In Figure 36 all of the data points have been compiled to reflect the effects of a) WT and b) surface finish, on amorphous content (proportional to crystallization peak area). 
It can be readily seen that both factors significantly impact the total amorphous fraction in the samples. With a wheel temperature of $200^{\circ} \mathrm{C}$, the average crystallization peak size was increased by nearly a factor of 2 over samples meltspun on a wheel held at either room temperature or $100^{\circ} \mathrm{C}$. The amorphous content also decreased continuously with increasing grit size (decreasing abrasive particle diameter).
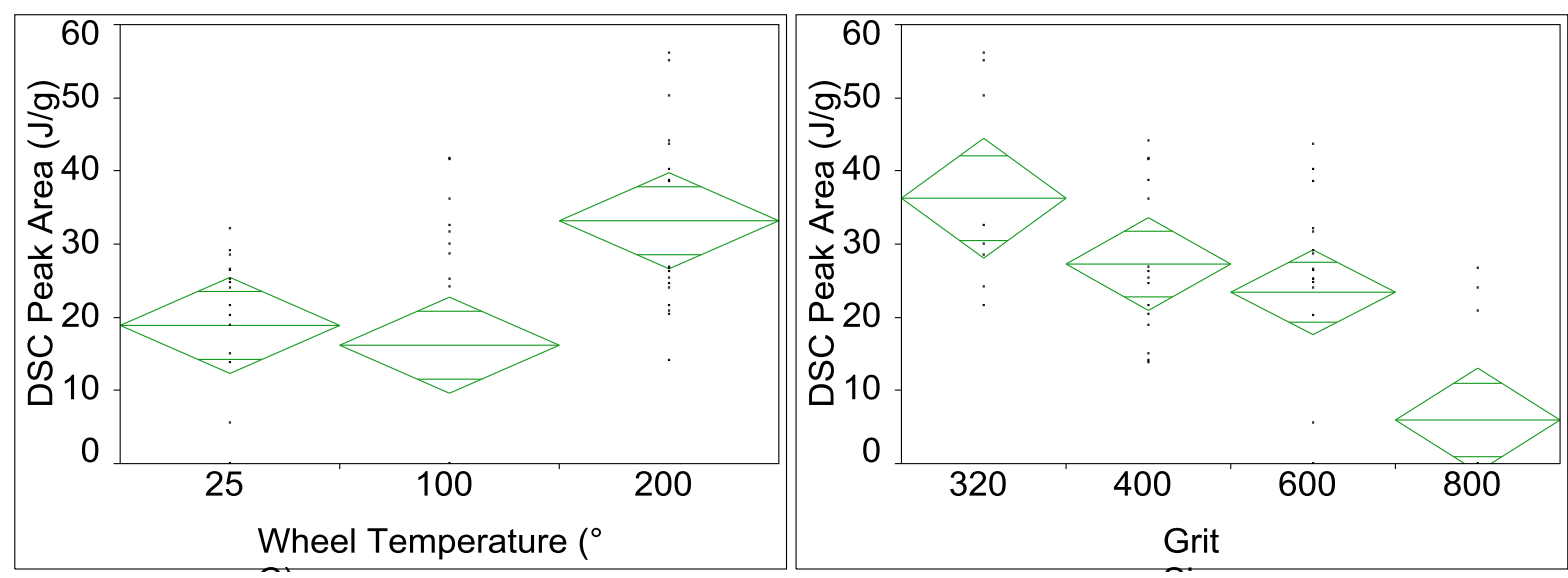

Figure 36 - Differential scanning calorimetry crystallization peak area plotted as a function of a) wheel temperature, and b) abrasive grit size used to polish the wheel. The centerlines of the diamonds represent population means and the points represent $95 \%$ confidence intervals.

\section{Optical Profilometry}

The wheel-side surfaces of the ribbons were characterized using a Zygo

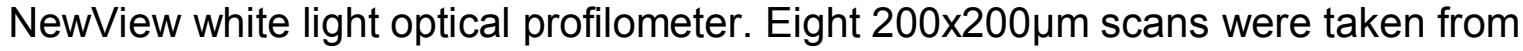
each batch of ribbons. An example profile is shown in Figure 37. From the data, tenpoint mean roughness values $\left(R_{z}\right)$ were calculated (see Figure 38 ). This parameter is calculated by summing the 5 greatest height peaks, subtracting the 5 lowest height valleys, and dividing by 5 . Wheel temperature was seen to have no statistically significant effect on roughness, while the 320 grit surface finish was seen to have significantly higher roughness than the other two finer grits that were characterized with the profilometer. 


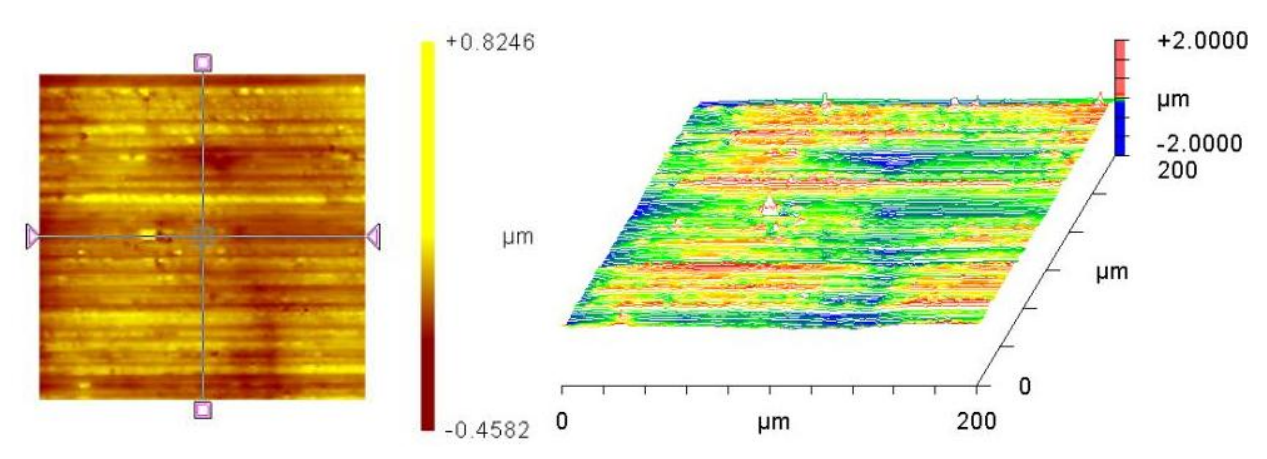

Figure 37 - Optical profilometer scan of $\mathrm{Nd}_{4} \mathrm{Fe}_{28} \mathrm{~B}_{2} \mathrm{TiC}$ ribbon melt-spun at $15 \mathrm{~m} / \mathrm{s}$ on a $25^{\circ} \mathrm{C}$ wheel polished to 600 grit.

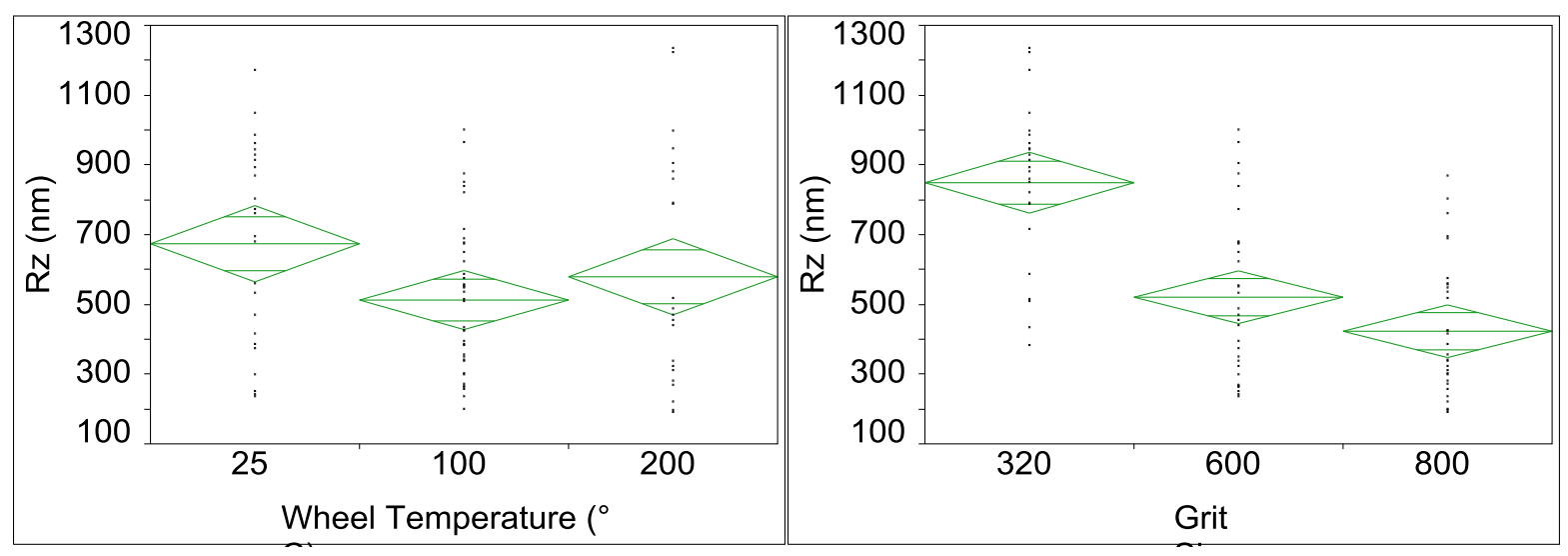

Figure 38 - Ten-point mean roughness (Rz), as measured by $200 \times 200 \mu m$ optical profilometer scans. Data is plotted by a) wheel temperature and b) wheel surface finish.

\section{Optical and Scanning Electron Microscopy}

Optical micrographs were taken of the wheel sides of several ribbons from each batch. Once the liquid cools and solidifies, a solid-solid interface between the ribbon and wheel forms. This interface forms a template of the wheel surface, depending on the influences of wetting and gas entrapment that will be discussed. Grooves run along the circumference of the wheel, a by-product of the polishing step used to clean the wheel between runs. These grooves are transferred to the ribbon in regions where good contact (wetting) occurs between the ribbon and wheel.

In the optical images, three different regions became apparent: regions where the melt wetted the wheel well, regions where the melt wetted the wheel 
poorly, and regions were there was a high density of entrapped gas between the wheel and the melt. Figure 39 shows an optical image of a piece of ribbon where both regions of good wetting and poor wetting (indicated by the arrows) can be seen. Figure 40 shows a backscattered electron image of the transition from good wetting to poor wetting regions. An arrow denotes the transition boundary. Note that the wheel striations can still be seen in the ribbons, but the features appear blurred. This could be explained by the schematic in Figure 41 . If the melt does not wet the wheel fully, the reproduction of the wheel surface loses contrast.

The relative amounts of each type of region were measured over 15 different images from each batch using ImageJ software [85]. The fraction of poor contact area is plotted in Figure 42 vs. a) wheel temperature, and b) wheel surface finish. Again, wheel temperature does not exhibit a consistent trend, unlike the apparent rising trend with smaller polishing grit size.

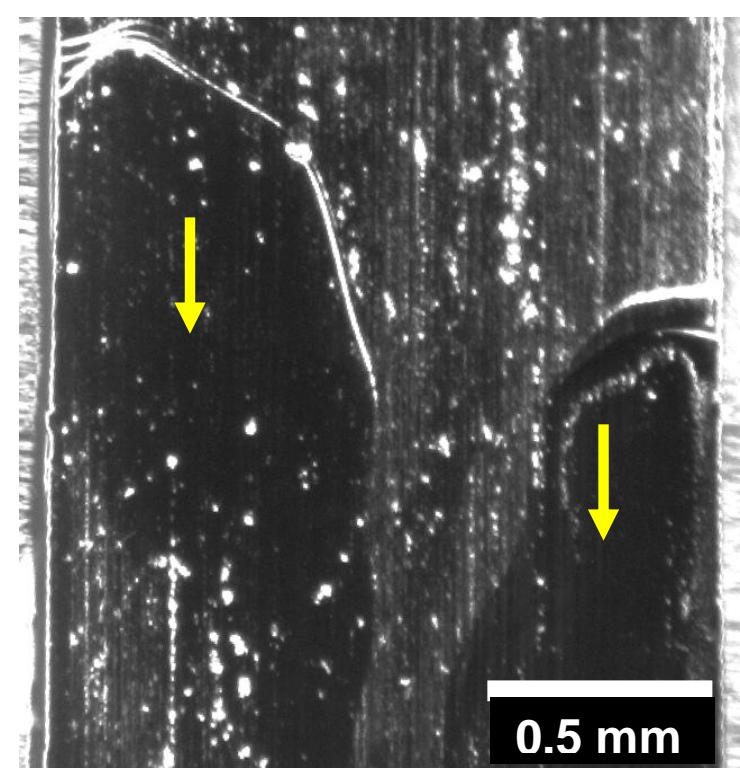

Figure 39 - Optical micrograph of the wheel side of a ribbon piece melt-spun on a $100^{\circ} \mathrm{C}$ wheel using 600 grit abrasive paper to polish the wheel. The arrows point to regions where contact between the wheel and the melt were poor. 


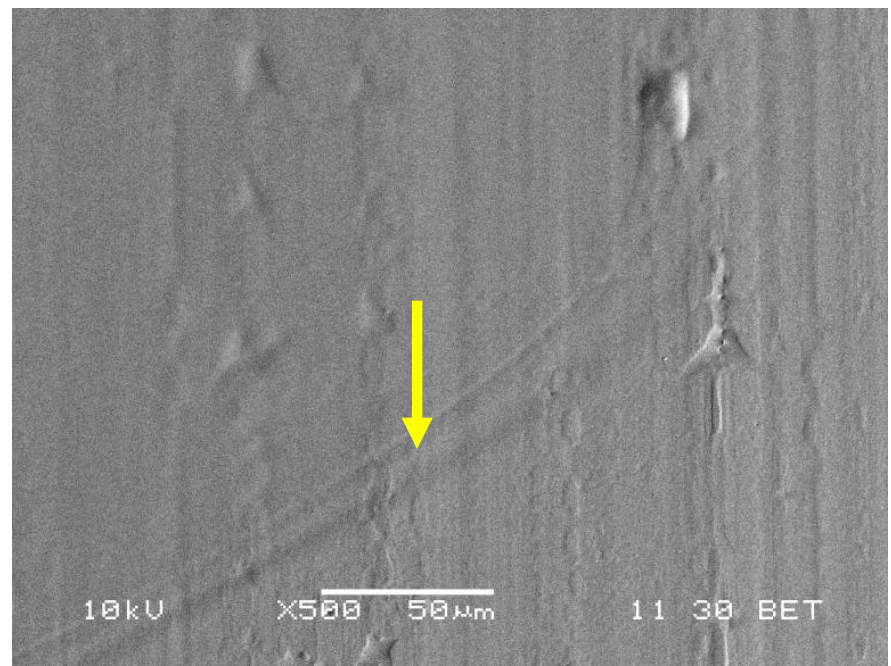

Figure 40 - Backscattered electron image of a transition from good wetting to poor wetting regions. The boundary is marked with an arrow.
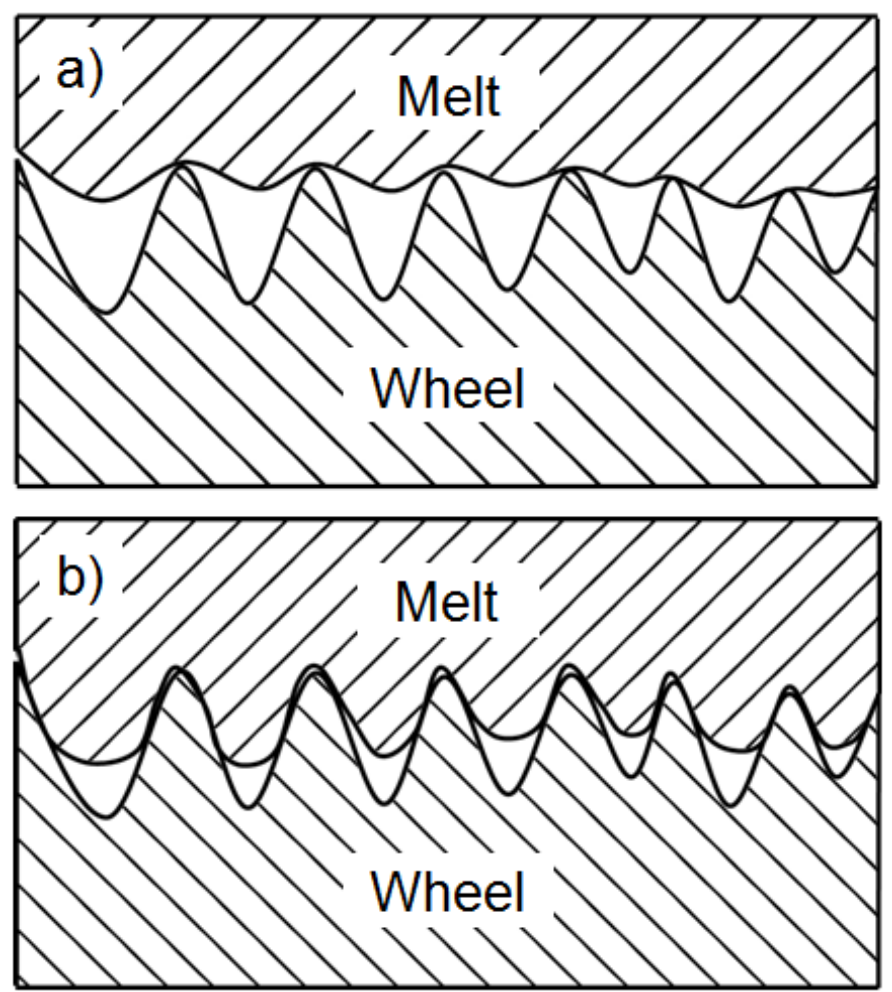

Figure 41 - Schematic of melt/wheel interface for a) poor wetting region b) good wetting region. 

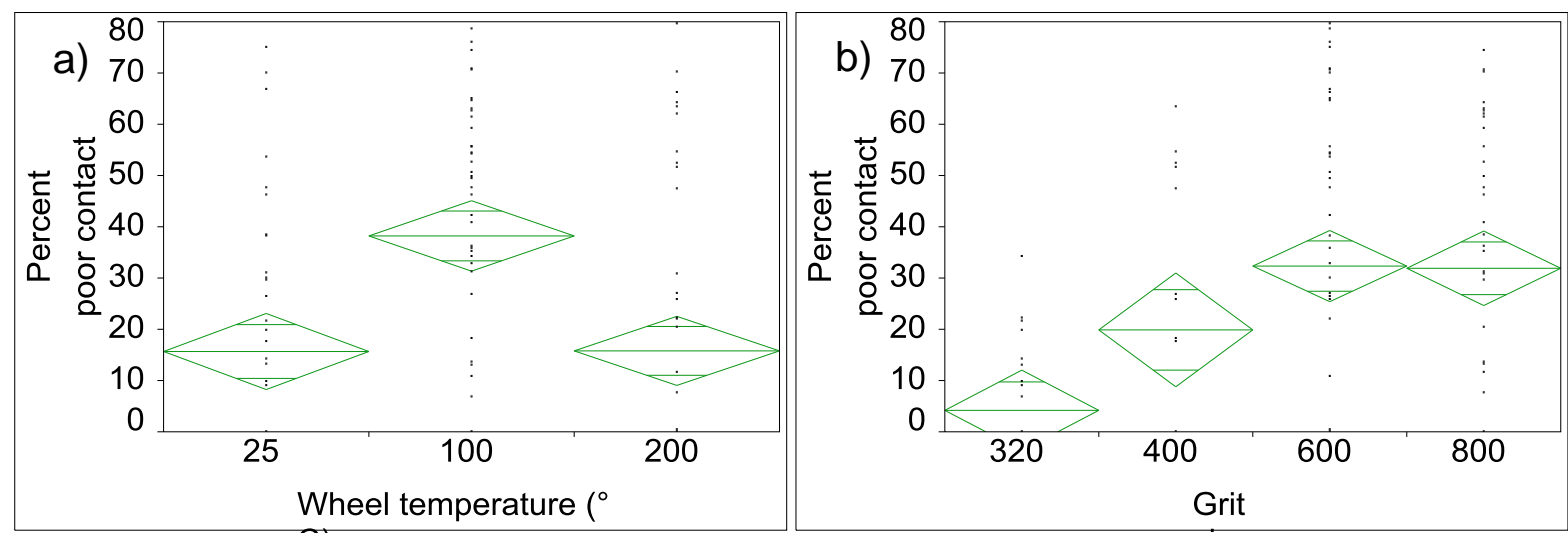

Figure 42 - Relative amount of poor contact region (as determined by ImageJ analysis of optical micrographs) as a function of a) WT and b) surface finish.

Entrapped gas regions were found in all batches of ribbon. These regions made up approximately $15 \%$ of the total area of ribbon surveyed. However, no statistically significant differences were found when either the WT or surface finish was varied. Figure 43 shows a region where a high concentration of entrapped gas pockets was seen.

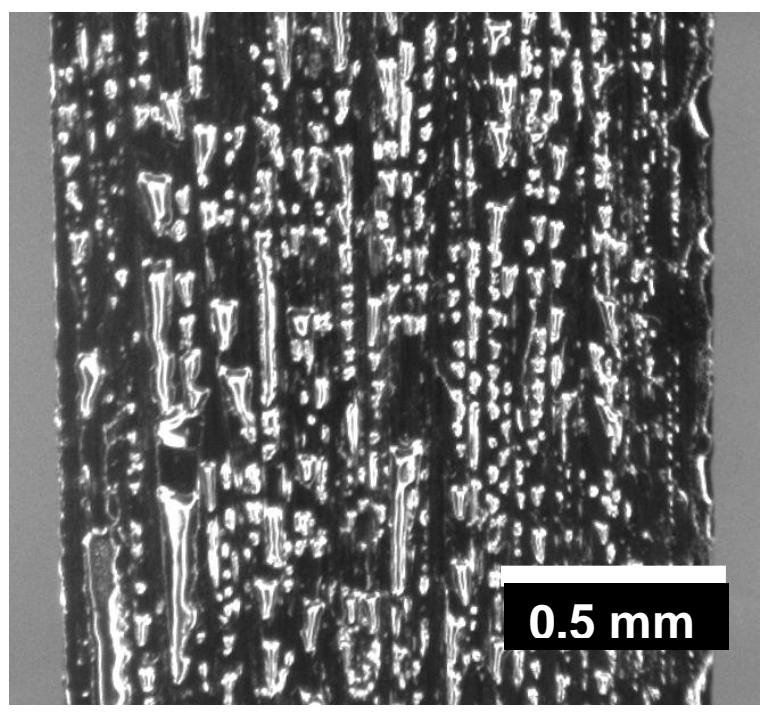

Figure 43 - Optical micrograph of the wheel side of a ribbon piece melt-spun on a $100^{\circ} \mathrm{C}$ wheel using 600 grit abrasive paper to polish the wheel. Surface features indicate a high degree of entrapped gas pockets. 


\section{Discussion}

Heat Flow Considerations

The data presented above clearly indicate that modification of surface finish and WT result in significant changes in microstructure and amorphous phase selection upon solidification at moderate wheel speeds (around $15 \mathrm{~m} / \mathrm{s}$ ). While the effects of increasing wheel temperature to $200^{\circ} \mathrm{C}$ that increase the formation of glassy phase may seem counterintuitive (most models indicate that increased temperature of the wheel would result in reduced temperature gradient and, therefore, have a negative impact on heat extraction rate $[84,86,87])$, this increased amorphous phase selection can be explained by other enhancements of the heat flow between the melt and the wheel.

The x-ray diffraction data shows evidence of three different phases upon solidification of the ribbons: amorphous phase, $\mathrm{Nd}_{2} \mathrm{Fe}_{14} \mathrm{~B}$, and $\alpha-\mathrm{Fe}$. Depicting the conditions necessary for these phases to form can be presented through a timetemperature-transformation (TTT) diagram, or continuous cooling transformation (CCT) diagram. The CCT diagram depicts the amount of time necessary to form a given phase (either partially or wholly) based on the thermal cooling profile. These diagrams are largely affected by the kinetics of the system. To be useful, it must be assumed that the nucleation kinetics have not been affected by factors such as changes in composition or the number of heterogeneous nucleation sites. At very slow cooling rates, the system approaches thermodynamic equilibrium conditions. At higher cooling rates, nucleation and growth of certain phases can be suppressed or avoided entirely. A schematic CCT diagram, similar to one created by Branagan et al.[88] for such magnet alloy ribbons, provides a useful tool to map phase formation based on ribbon cooling conditions (see Figure 44).

The sample cooling curves on the diagram show three different regions. Initially, very high cooling rates are seen when the liquid melt is in direct contact with the wheel surface and essentially all heat is withdrawn through conduction to the wheel. At an intermediate time, a solid layer forms between the liquid and the wheel (as seen in Figure 45), reducing the conductive heat flow rate and allowing top 
surface radiative heat flow to split the heat flow directionality. The final region represents the point at which the ribbon separates from the wheel surface and cooling is fully bifurcated to top and bottom surfaces through radiation and convection within the chamber atmosphere.

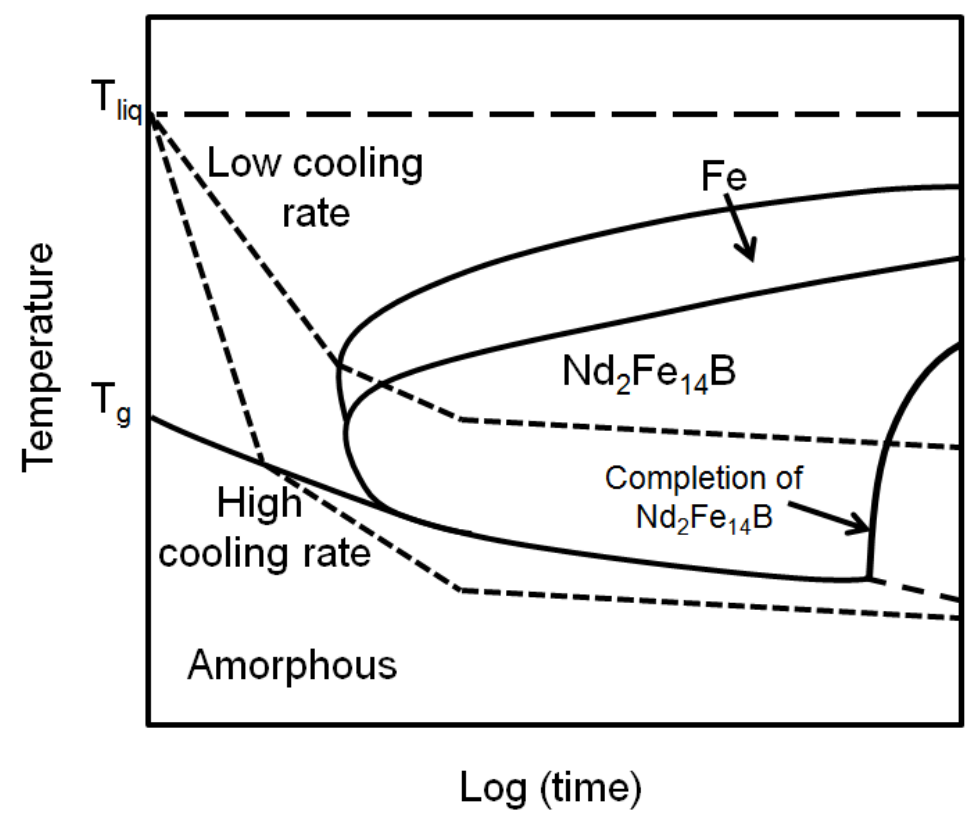

Figure 44 - Schematic TTT diagram for high cooling rates in $\mathrm{Nd}_{2} \mathrm{Fe}_{14} \mathrm{~B}$. The cooling curves depicted show 3 regions representing cooling while the melt is liquid on the wheel, once a solid layer is present in the melt, and once the ribbon has parted from the wheel surface.

Since this work focuses on rapidly solidified materials, the left side of the diagram is of primary concern. At high cooling rates, diffusion is slowed to such a degree that there is insufficient time to nucleate any crystalline phase, resulting in a fully amorphous structure (schematically represented by the dashed line marked "High cooling rate" on the TTT diagram). While this is a likely explanation for complete glass formation (from XRD and DSC evidence) in the samples melt-spun at 20 and $25 \mathrm{~m} / \mathrm{s}$ on a $200^{\circ} \mathrm{C}$ wheel, the $25^{\circ} \mathrm{C}$ wheel never did produce fully amorphous ribbon, even at the highest wheel speeds. At lower cooling rates, Fe and $\mathrm{Nd}_{2} \mathrm{Fe}_{14} \mathrm{~B}$ will nucleate and grow sequentially, resulting in fully crystalline material (represented by the dashed line marked "Low cooling rate"). This was observed in 
the samples melt-spun at $10 \mathrm{~m} / \mathrm{s}$ at both $25^{\circ} \mathrm{C}$ and $200^{\circ} \mathrm{C}$ wheel temperatures and $15 \mathrm{~m} / \mathrm{s}$ in the $25^{\circ} \mathrm{C}$ wheel (see Figure 34). At cooling rates between those described above, some glassy phase will form initially, but both $\mathrm{Nd}_{2} \mathrm{Fe}_{14} \mathrm{~B}$ and Fe nuclei form, producing all 3 solidification phases after cooling. Therefore, it should be emphasized that fully amorphous ribbons formed at moderate (only $20 \mathrm{~m} / \mathrm{s}$ ) wheel speeds in spite of lowering the melt/wheel surface temperature gradient (by raising the wheel temperature to $200^{\circ} \mathrm{C}$ ). This means that some other influences must have operated to promote a high cooling rate and bypass of crystallization, assuming no changes in the nucleation kinetics.

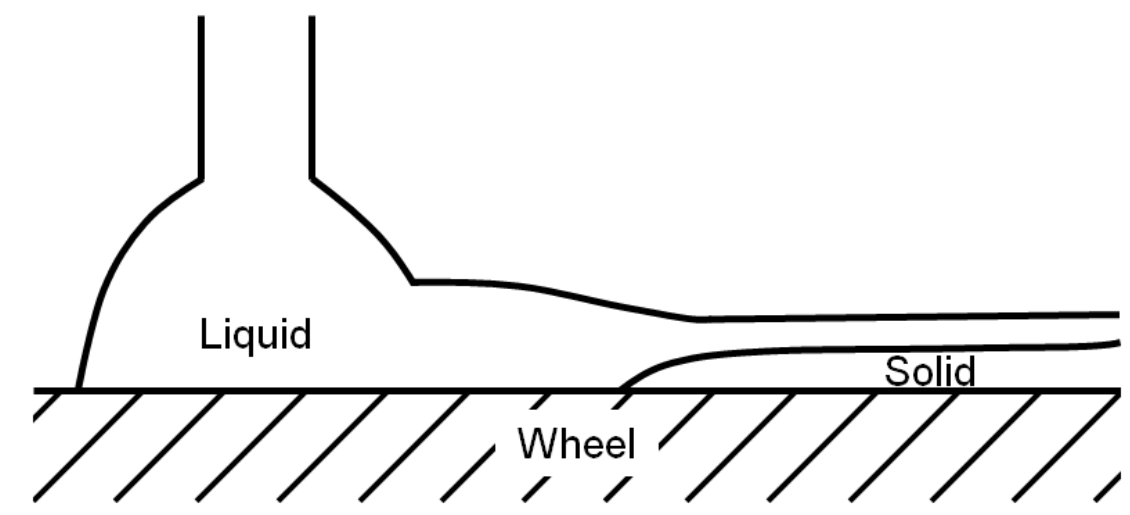

Figure 45 - Melt pool schematic. The motion of the wheel surface is to the right.

If a decrease in ribbon thickness were observed for a high WT, one could argue that the increased cooling rate was a function of decreased thermal mass. However, no significant change in ribbon thickness is observed for WT of $25^{\circ}$ and $200^{\circ} \mathrm{C}$, indicating that the increase in glass forming tendency for WT of $200^{\circ} \mathrm{C}$ is likely caused by increase of conductive heat transfer into the wheel. This change induced by an increased WT must be caused by some means of enhancing the interfacial heat transfer coefficient at the wheel-melt interface. As previously mentioned, a higher wheel temperature decreases the temperature difference between the melt and the wheel surface, resulting in a lower thermal gradient and a decrease in heat flow, if nothing else changes (i.e. thermal conductivity or contact 
area) in the conductive heat transfer equation (Fourier's law of conduction, shown below).

$$
q=-k A \nabla T
$$

Where $q$ is the heat flow (in W), $k$ is the thermal conductivity (in $W / m-K$ ), and $\nabla \mathrm{T}$ is the thermal gradient (in $\mathrm{K} / \mathrm{m}$ ).

However, thermal gradient is likely not the dominant factor for melt spinning of this magnet alloy since the resulting heat flow appears to be increased at the highest wheel temperature observed, not decreased.

Another factor that can affect heat flux is thermal conductivity of the wheel. As temperature is increased, thermal conductivity in copper is decreased, which should produce a reduced heat flow, again counter to the observations. However, the thermal conductivity of copper, even at $200^{\circ} \mathrm{C}$, is so much greater (by approximately an order of magnitude[89]) than the thermal conductivity of the other phases in the system, relatively little contribution to a decreased heat flux would be expected.

Heating the wheel may also alter the phases present in the melt while the ribbon is in contact with the wheel. For example, if a solid layer (e.g., a glassy layer) forms between the wheel and the liquid, an additional interface (and the thermal resistance associated with it) must be considered to add heat flow resistance. Additionally, differences in the thermal conductivity between the liquid and the solid likely exist. The thermal conductivity of crystalline $\mathrm{Nd}_{2} \mathrm{Fe}_{14} \mathrm{~B}$ is very low $(\sim 7$ $\left.\mathrm{W} / \mathrm{m}^{*} \mathrm{k}\right)$ [90]. For comparison, the thermal conductivity of liquid $\mathrm{Fe}$ is on the order of $30 \mathrm{~W} / \mathrm{m}^{*} \mathrm{k}[89]$. As the melt solidifies, thermal contraction of the melt can also cause it to pull away from the wheel, degrading the interface and further reducing the interfacial heat transfer coefficient [91].

The most likely cause of the observed changes in amorphous content with different WT is the "condition" of the interface between the wheel and the melt, e.g., wetted contact area and wheel surface topology and surface compounds. This is 
partially evidenced by the trends in crystallization peak area (see Figure 36) that mirror the changes in surface preparation condition (i.e., grit size) for each ribbon batch (see Figure 42). In other words, heat transfer can still occur across non-wetted gaps through convection and radiation in a poor contact region, but the efficiency is reduced. By improving interfacial wetting and contact area, the interfacial heat transfer can be increased, for example. While the exact blend of phenomena that enhance the interfacial thermal conductivity is not yet clear, possible explanations can be derived from the effects on glass formation for changes in both the wheel surface finish and the wheel temperature.

Using coarser grit sizes to polish the wheel can result in larger grooves for the melt to fill, possibly increasing the available surface area for heat to be transferred across. The deeper grooves may also permit an avenue for entrapped gas to escape, allowing for better conformity between the melt and the wheel.

Surface tensions of molten metals are dependent on temperature [92], as well as viscosity, especially for glass forming alloys [93]. By heating the wheel and lowering the thermal gradient, the liquid can remain at higher temperature (and, therefore, lower viscosity and surface tension) for a longer period of time, again allowing for greater conformity of the melt to the wheel surface, as illustrated in Figure 41. As a result, greater heat extraction rates from the melt would be achievable.

Wetting behavior can also be affected by the presence of an oxide on the wheel surface [94, 95]. Interestingly, copper has been shown to have somewhat complex oxidation behavior at low temperatures, displaying at least 3 different oxides in the temperature range of room temperature to $330^{\circ} \mathrm{C}$ in an air atmosphere $[96,97]$. From room temperature to approximately $70^{\circ} \mathrm{C}$, little oxide is formed, but the presumed phase is $\mathrm{Cu}_{2} \mathrm{O}$. From $70^{\circ} \mathrm{C}$ to $110^{\circ} \mathrm{C}, \mathrm{Cu}_{2} \mathrm{O}$ grows significantly. $\mathrm{Cu}_{3} \mathrm{O}_{2}$, a highly defected $\mathrm{Cu}_{2} \mathrm{O}$ structure grows from $110^{\circ} \mathrm{C}$ to about $200^{\circ} \mathrm{C}$. At temperatures above $200^{\circ} \mathrm{C}, \mathrm{CuO}$ is the primary oxide. However, the oxidation behaviors at low partial pressures of oxygen (as is the case in the experiments presented here) have not been well studied. Thus, it is possible that different oxide phases might be 
present at different wheel temperatures. Most importantly, these different oxide phases may have significantly different wettability for the magnet alloy melt and, therefore, much different contact area for heat transfer. Further study on the effects on wetting behavior of the different oxide stoichiometries and, perhaps, oxide thicknesses on the Cu wheel surface are needed to test this. Another obvious experiment might be to switch the type of metal on the wheel surface, e.g., by applying a Ni plating, and, then, to perform the same series of experiments with WT changes.

\section{Ribbon Adhesion to the Wheel}

As another way to gain understanding of the changes in the wheel condition, several of the runs were filmed using a Vision Research Phantom v7.1 high speed camera. On runs using the heated wheel and higher wheel speeds $(>20$ $\mathrm{m} / \mathrm{s}$ ), initially, a constant melt stream and melt pool were observed (see Figure 46). Partway through the run, ribbon was seen to adhere to the wheel surface, indicating the effect of improved wetting. The adhered, solidified ribbon disrupted the melt stream, causing splashing and initiating an arc from the induction coil (see Figure 47). To explain the later onset of ribbon adherence, it is likely that, upon successive rotations, a track on the wheel heats to even greater temperatures. If the ribbon conforms to the wheel better at higher wheel temperatures, greater increases in WT may result in adhesion to the wheel. Further, these types of observations showed that the apparent effect of improved wetting with the $200^{\circ} \mathrm{C}$ wheel also was exacerbated by utilizing coarser grits to finish the wheel. While heating the wheel and using coarser finishes can improve the quench obtained, too much improvement of wetting can also cause excessive adhesion of the ribbon and disruption of the melt stream, showing the need for careful monitoring and control of the wheel temperature, for example, during extended runs. 


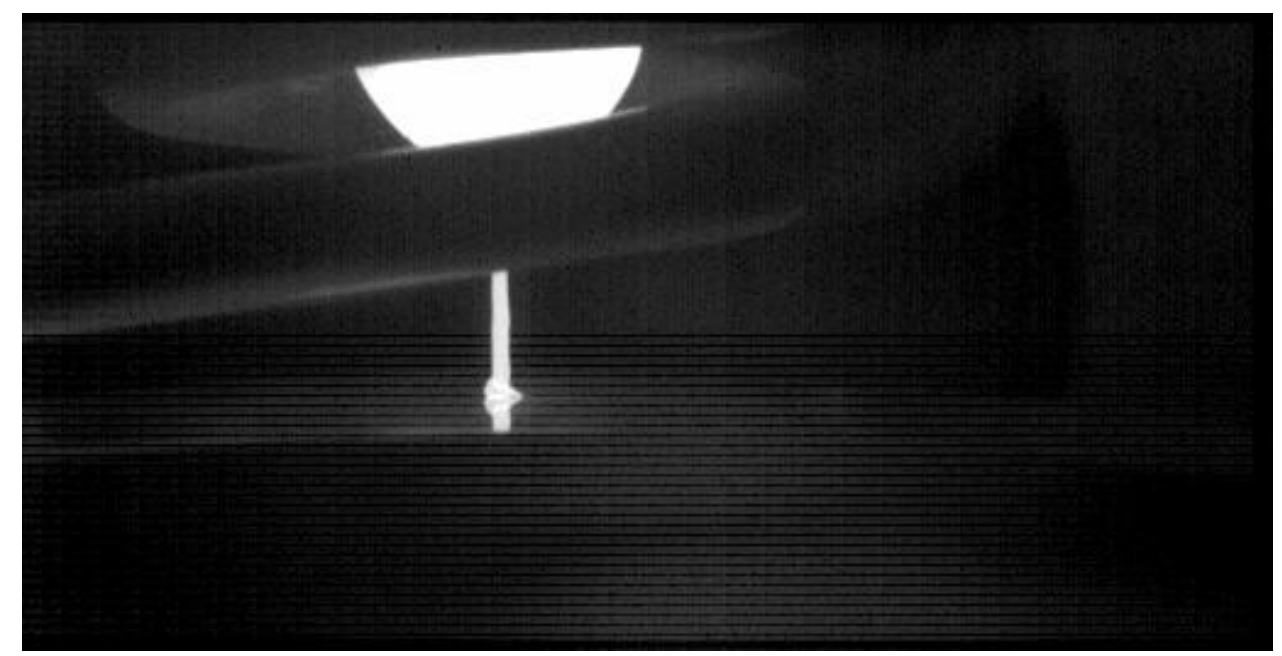

Figure 46 - Melt stream image taken with a high speed camera. The wheel was rotating to the right.

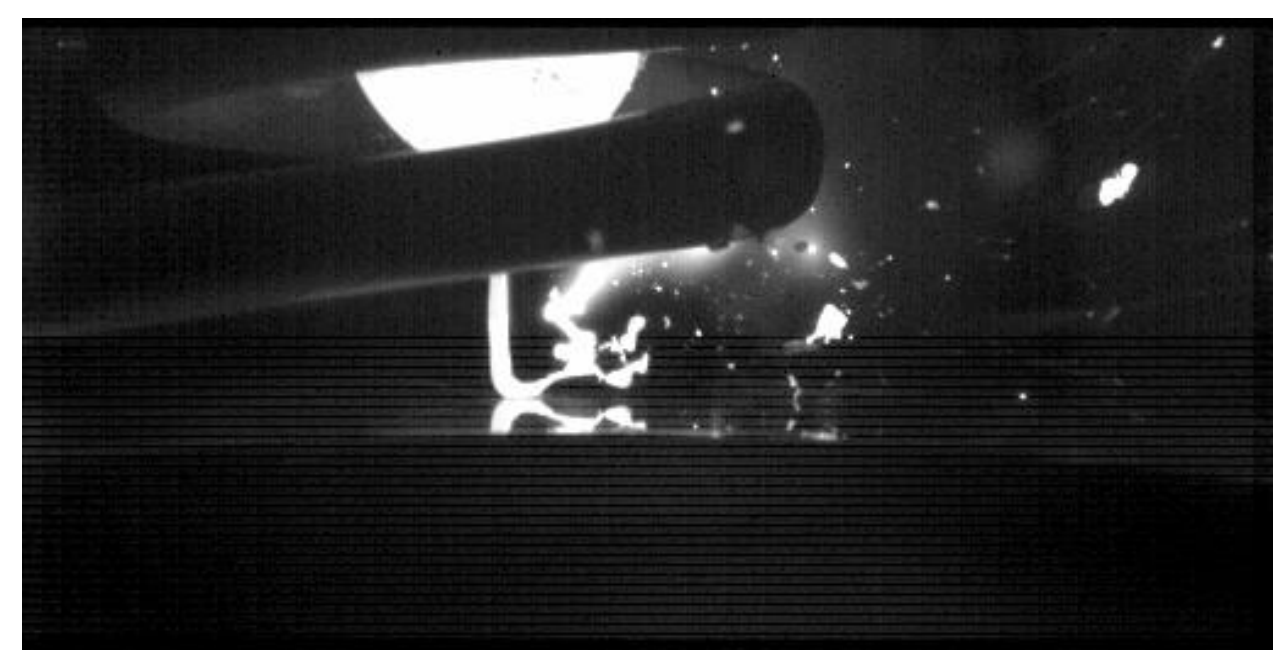

Figure 47 - Melt stream image once ribbon began adhering to the wheel surface.

\section{Conclusions}

- Melt-spinning onto a $200^{\circ} \mathrm{C} \mathrm{Cu}$ wheel was found to increase the amount of amorphous phase present (as determined by DSC and XRD) and, presumably, 
heat flow between the melt and wheel in Fe-11Nd-6B-3Ti-3C (at\%) ribbon relative to melt-spinning on a room temperature or $100^{\circ} \mathrm{C}$ wheel.

- In the room temperature wheel, fully amorphous samples were never achieved, while fully amorphous samples could be achieved at 20 and $25 \mathrm{~m} / \mathrm{s}$ using the $200^{\circ} \mathrm{C}$ wheel.

- Amorphous content (and heat flow between the melt and wheel) were seen to decrease when the wheel was surfaced using finer grit abrasive paper (from 320 to 800 grit).

- The amorphous content was found to correlate with surface roughness (as measured by optical profilometry) and surface wetting (as determined by optical microscopy), indicating overall heat flow is partially dictated by the wetting behavior between the melt and the wheel.

- Further evidence of the enhanced wetting was seen as ribbon pieces adhered to the wheel, particularly at high wheel temperature and coarse grit surface finishes.

- While the exact phenomena resulting in enhanced wetting by the elevated WT is not yet known, possible explanations for the enhanced wetting include delay of increased surface tension or viscosity of the melt (resulting from the melt staying at a higher temperature for a longer period of time) and modification of the oxide compound on the copper wheel surface.

\section{Acknowledgements}

This work was supported by the Department of Energy-Energy Efficiency and Renewable Energy, Vehicle Technology Office, US-DRIVE program, under Contract No. DE-AC02-07CH11358 for the operation of Ames Laboratory (USDOE). 
The assistance of Nicholas Fries, Allison Loecke, and Eric Harms in sample preparation and characterization is greatly appreciated. Optical profilometry data collection was completed at Arnprior Rapid Manufacturing Solutions. 


\section{Chapter 6 - Effects of $\mathrm{Ag}$ additions on melt-spun $\mathrm{RE}_{2} \mathrm{Fe}_{14} \mathrm{~B}$ microstructure and texture}

A paper published in the Journal of Applied Physics

N.T. Oster ${ }^{1,2}$, D.T. Cavanaugh ${ }^{2}$, K.W. Dennis ${ }^{2}$, M.J. Kramer ${ }^{1,2}$, R.W. McCallum ${ }^{1,2}$, I.E. Anderson ${ }^{1,2}$

${ }^{1}$ Department of Materials Science \& Engineering, lowa State University, Ames, IA 50011, USA

${ }^{2}$ Materials and Engineering Physics, Ames Laboratory, Ames, IA 50011, USA

\section{Abstract}

Control of microstructure and texture is of critical importance in forming rare earth $(\mathrm{RE})$-iron-boron particulate suitable for anisotropic polymer-bonded permanent magnets and anisotropic sintered permanent magnets. In this study, the selected approach to controlling grain size, while maintaining texture, is through stabilization and refinement of directional growth in melt-spun ribbons. Varying concentrations of $\mathrm{Ag}$ were added to melt-spun ribbons of composition $\left(\mathrm{Y}_{0.55} \mathrm{Nd}_{0.45}\right)_{2.2} \mathrm{Fe}_{14} \mathrm{~B}_{1.1}$. Effects on microstructure and texture were observed through scanning electron microscopy (SEM) and $x$-ray diffraction (XRD). It was determined that Ag stabilized columnar growth (compared to alloys with no Ag added) with additions as small as 0.3 at. \%, but the $\mathrm{Ag}$ also produced a unique texture in the ribbons. In RE-Fe-B ribbons without $\mathrm{Ag}$, strong $<00>$ texture is observed at the free surface and a mechanism has been established. In all Ag-containing ribbons, the observed texture is canted to both the $\mathrm{c}$ - and a-axes, but the mechanism remains unclear.

\section{Introduction}

Rare earth ( $\mathrm{RE}$ )-iron-boron permanent magnet (PM) alloys, primarily consisting of the structure $\mathrm{RE}_{2} \mathrm{Fe}_{14} \mathrm{~B}$, are widely used in applications where high performance PMs are required [11]. In order to obtain the highest performance from 
these alloys, anisotropic material is required. Especially in the case of polymerbonded magnets, anisotropic magnets would have a great advantage over current isotropic bonded magnets, up to $4 \mathrm{X}$ the maximum energy product. Particulate for these applications must be able to maintain coercivity as well as large enough particle size to avoid excessive oxidation. Suitable particulate for isotropic polymerbonded magnets for use at high temperatures $\left(\sim 200^{\circ} \mathrm{C}\right)$ has been realized through the addition of yttrium and heavy rare earth elements (i.e. Dy) [98]. Concerns over price and availability of heavy rare earths have led us to experiment on Dy-free alloys.

One avenue to obtain suitable anisotropic particulate is through stabilization of directional growth in melt-spun ribbons, followed by mild crushing (a low-melting transition metal coating may be needed to reduce reverse domain nucleation sites on fracture surfaces). The solidification pathways for these materials have been previously documented, both from single crystals ${ }^{3}$ and melt-spun ribbons ${ }^{4}$. Single crystal growth experiments by Tenaud et al. [99] have shown that the a-axes of the tetragonal unit cell are the preferred growth directions. However, Kramer et al. [59] determined that ribbons melt-spun at low wheel speed (e.g. 5-10 $\mathrm{m} / \mathrm{s}$ ) displayed $\mathrm{a}<00 />$ texture on the free surface. This occurs since BCC-Fe is the primary solidification phase, not $\mathrm{RE}_{2} \mathrm{Fe}_{14} \mathrm{~B}$. Fe dendrites grow perpendicular to the wheel surface in the $<100>$ directions. When the alloy cools below the peritectic temperature, the $\mathrm{RE}_{2} \mathrm{Fe}_{14} \mathrm{~B}$ epitaxially templates to the iron dendrites and grows in its basal plane directions via the relationship [110](110)a-Fe $\|[110](006) 2-14-1$, resulting in the observed surface texture.

Since the columnar structure templates to the primary iron, refining the primary dendrite spacing of the iron could result in a finer microstructure that is needed for anisotropic bonded magnets. We hypothesized ${ }^{5}$ that this refinement might be achieved by using a segregating additive (such as $\mathrm{Ag}$ ) if the proper concentration was selected. Ag was chosen due to its insolubility in $\mathrm{Fe}$, the primary solidification phase, even into the liquid [100]. This study investigates the effects of Ag concentration on microstructure and the degree of texture in low wheel speed ( 5 
$\mathrm{m} / \mathrm{s}$ ) melt-spun ribbons, following Kramer et al. [59] We hypothesize that the primary Fe dendrite spacing could be refined if instability in the Fe-liquid interface can be introduced, resulting in a finer, textured structure.

\section{Experimental Procedure and Results}

Ingots of composition $\left(\mathrm{Y}_{0.55} \mathrm{Nd}_{0.45}\right)_{2.2} \mathrm{Fe}_{14} \mathrm{~B}_{1.1} \mathrm{Ag}_{\mathrm{x}}$, where $\mathrm{x}$ is varied from 0 0.3 (0-1.7 at. \%), were arc melted and cast on a water-cooled copper hearth, using a non-consumable tungsten electrode. The ingots were melt-spun at $5 \mathrm{~m} / \mathrm{s}$ tangential wheel velocity in a high purity He atmosphere $(0.33 \mathrm{~atm}$.) using a free jet meltspinner. The resulting as-spun ribbons were mounted, cross sectioned and polished. Backscattered electron (BSE) micrographs were taken using a JEOL 5910LV scanning electron microscope, as seen in Figure 26. All images are oriented such that the surface at the bottom of the image was in contact with the wheel (i.e. the hot-to-cold thermal gradient points downward). 

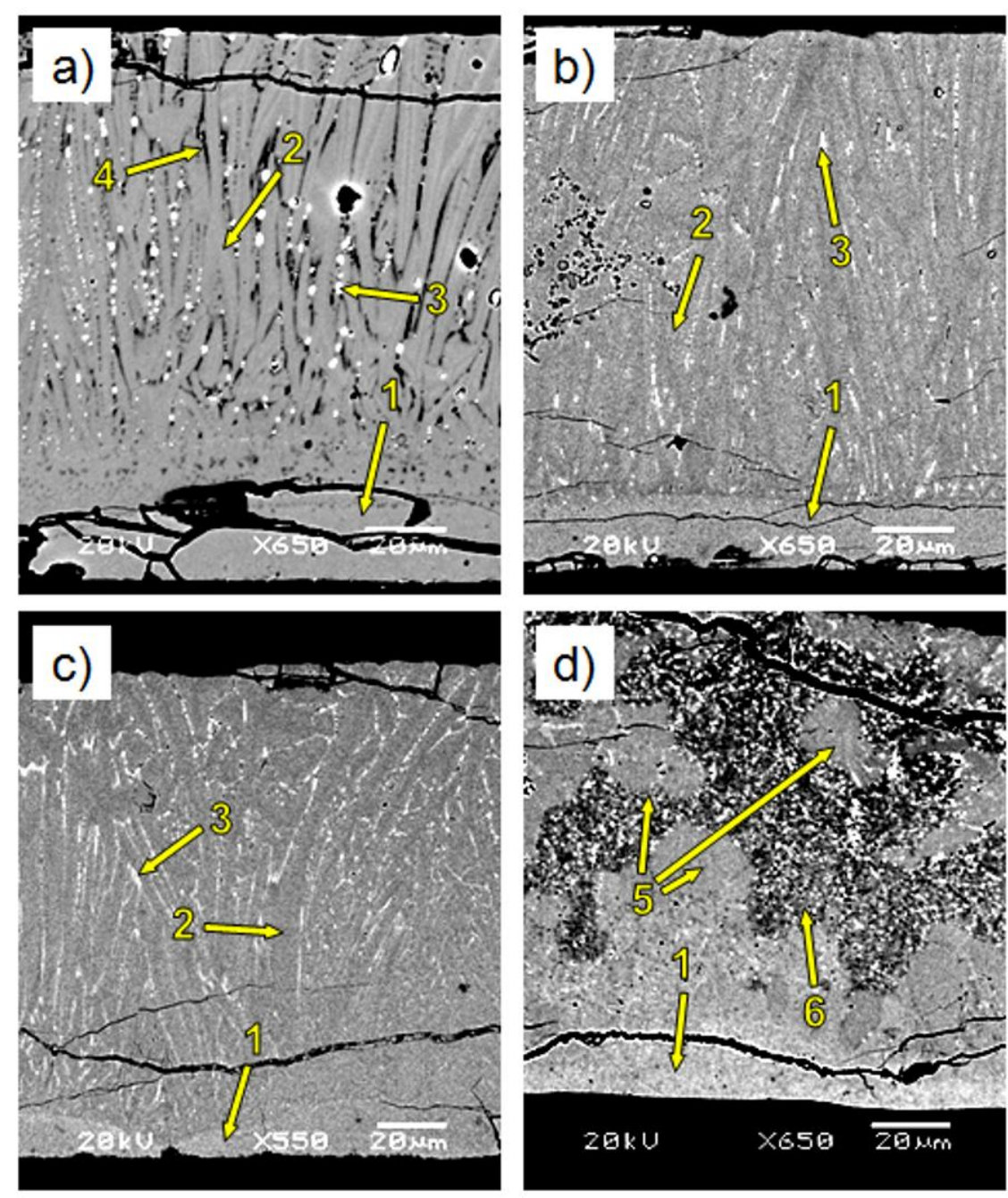

Figure 48 - Cross section BSE micrographs of $\left(\mathrm{Y}_{0.55} \mathrm{Nd}_{0.45}\right)_{2.2} \mathrm{Fe}_{14} \mathrm{~B}_{1.1} \mathrm{Ag} \mathrm{x}$ ribbon meltspun at $5 \mathrm{~m} / \mathrm{s}$, where $x$ is a) 0.3, b) 0.1 , c) 0.05 , or d) 0 . Arrows point to features described in the text.

In all alloys containing $\mathrm{Ag}$, a layer of fine equiaxed grains, approximately $20 \mu \mathrm{m}$ thick, was present near the wheel surface (identified in Figs. 26 a to $26 \mathrm{~d}$ by the arrows labeled "1") Growing out of this region, columnar dendrites of $\mathrm{RE}_{2} \mathrm{Fe}_{14} \mathrm{~B}$, with little secondary arm formation, grew through (essentially) the thickness of the ribbon (denoted by arrows labeled "2" in Figs. 26a to 26c). Alloys where $x \geq 0.1$ (0.6 at. \% $\mathrm{Ag}$ ) displayed a fine secondary phase, enriched in $\mathrm{Ag}$ and $\mathrm{Nd}$ (the bright spots labeled "3" in Figs. 26a to 26c), dispersed along the boundaries of the columns. Energy dispersive spectroscopy (EDS) of the parent ingots indicated that this phase 
is likely a $1: 1(\mathrm{Ag}: \mathrm{RE})$ intermetallic compound, with approximately $80 \%$ of the $\mathrm{RE}$ as $\mathrm{Nd}$, with the remainder $\mathrm{Y}$. This composition is consistent with a study of $\mathrm{Nd}-\mathrm{Fe}-\mathrm{Ag}$ equilibrium by Takeda et al. ${ }^{7}$ The morphology and distribution of the AgRE phase indicated that the phase likely coarsened during cooling. In the sample where $\mathrm{x}=$ 0.05 ( 0.3 at. \% Ag), a more continuous RE-rich film coated the columnar dendrites, similar to the intergranular phase observed in sintered $\mathrm{Nd}-\mathrm{Fe}-\mathrm{B}$ magnets.

In the sample with $0.3 \mathrm{Ag}$ (1.7 at. \%), a layer of Fe (labeled "4" in Fig. 26a) was also observed between the columns. This probably happened due to insufficient excess $\mathrm{RE}$ to fully bond with the $\mathrm{Ag}$ in the intermetallic, requiring $\mathrm{Nd}$ to be pulled from the 2-14-1 structure and leaving free Fe to form.

In samples containing no Ag, significant variation in microstructure exists. Some regions display a columnar microstructure similar to that observed in Fig. 26c. However, Fig. 26d shows a more typical microstructure. Along most of the wheel surface, the fine, equiaxed region exists. The columnar region is quickly interrupted by either large equiaxed grains (labeled " 5 " in Fig. 26d), or fine dendritic regions characterized by a large degree of secondary arm branching (labeled " 6 " in Fig. 26d). The bright phase in this micrograph corresponds to the RE-rich intergranular phase observed in typical sintered magnets.

Average center-to-center column spacing was determined using a lineintercept method. This was plotted as a function of distance from the equiaxed/columnar grain interface (where directional columnar growth initiates) to determine the degree to which columnar growth is stabilized and refined (Fig. 27). All cases show a fine grain spacing $(\sim 2 \mu \mathrm{m})$ near the interface between the equiaxed zone and the start of directional growth. The spacing slowly increases through the thickness of the ribbon. In the Ag-containing alloys, the concentration of $\mathrm{Ag}$ seems to have some effect on the stability of spacing of the columns, with $0.10 \mathrm{Ag}(0.6$ at.\%) as the most stable. However, an average error of $\pm 1.2 \mu \mathrm{m}$ adds uncertainty to this finding. In the case without silver, the columnar structure breaks down and grain spacing increases drastically as the columnar grains give way to larger, equiaxed ones. 


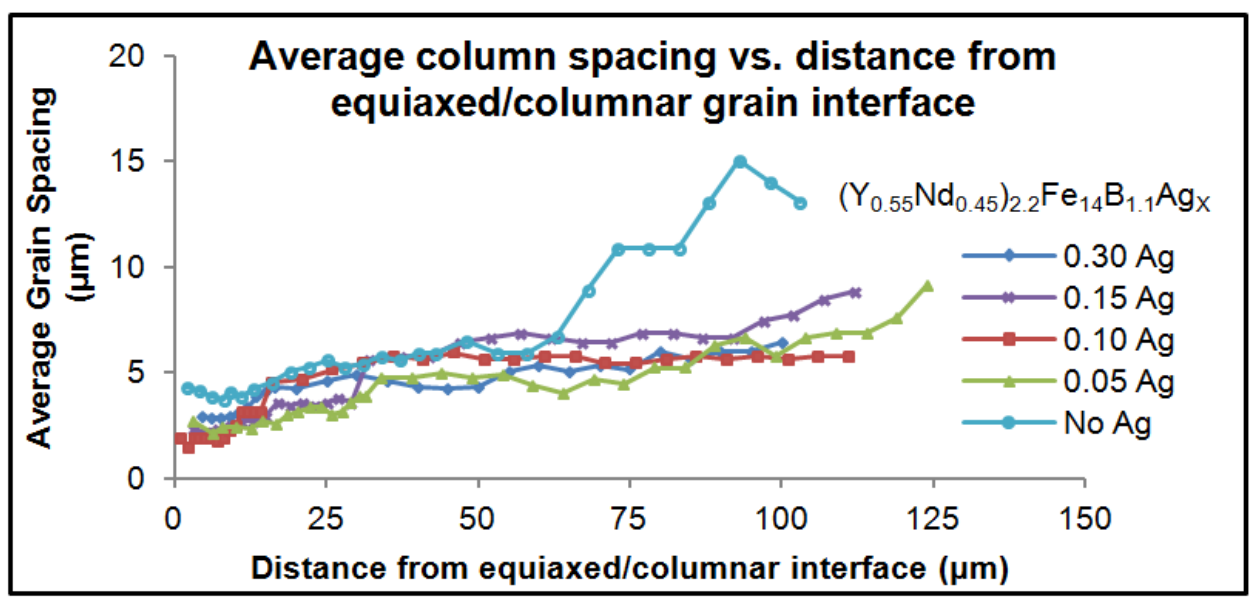

Figure 49 - Average center-to-center spacing of $\mathrm{RE}_{2} \mathrm{Fe}_{14} \mathrm{~B}$ columns as a function of distance from the fine, equiaxed region. Average error (calculated by $95 \%$ confidence interval) is $\pm 1.2 \mu \mathrm{m}$.

X-ray diffraction patterns of the ribbons were taken using a Panalytical $X$ 'Pert PRO diffractometer with Co K- $\alpha$ radiation. Ribbons of composition $\left(\mathrm{Y}_{0.55} \mathrm{Nd}_{0.45}\right)_{2.2} \mathrm{Fe}_{14} \mathrm{~B}_{1.1} \mathrm{Ag}_{0.15}$, were crushed and scanned (see Fig. 28). Rietveld refinement was done using General Structure Analysis System (GSAS) [101] software. The pattern matched well with a calculated isotropic trace for the $\mathrm{RE}_{2} \mathrm{Fe}_{14} \mathrm{~B}$ structure. One additional peak was observed at $0.379 \AA^{-1}$ inverse d-space (marked by a triangle in Figs. 28 and 29b). This peak corresponds well with the strongest peak in the AgRE phase (B2 structure), but is not enough, by itself, to confirm definitively the AgRE phase. No additional peaks, including BCC Fe, were observed. 


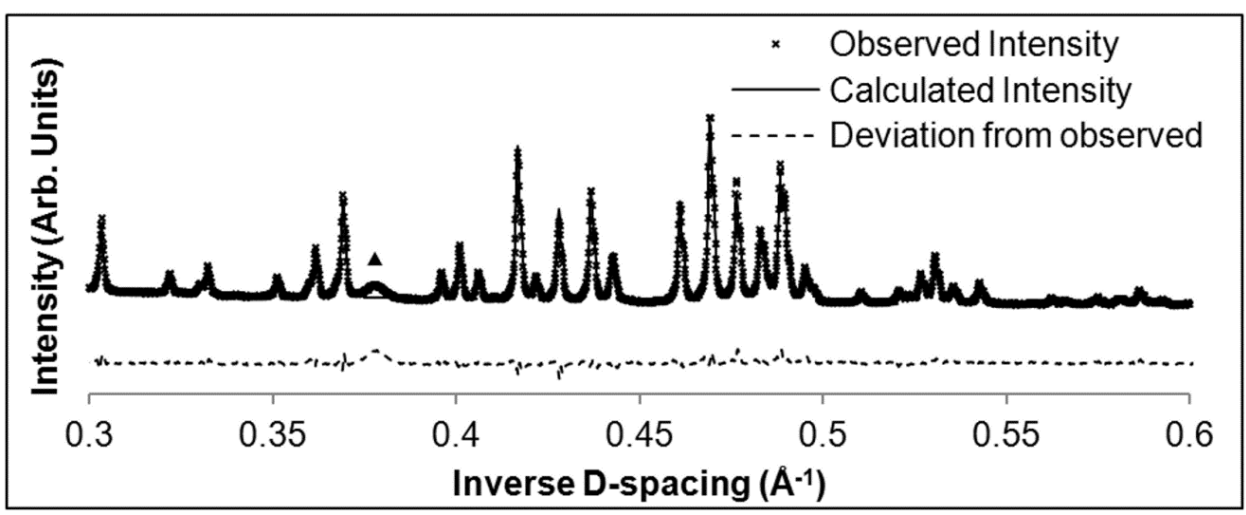

Figure 50 - XRD pattern for crushed $\left(\mathrm{Y}_{0.55} \mathrm{Nd}_{0.45}\right)_{2.2} \mathrm{Fe}_{14} \mathrm{~B}_{1.1} \mathrm{Ag}_{0.15}$ ribbon and accompanying Rietveld refinement trace for an isotropic powder pattern. The triangle marks the location of the only clear "extra" peak that corresponds to the most intense peak of the AgRE (B2 structure) phase.

To determine texture of each ribbon batch, a set of about 10 ribbon pieces, with the free surface facing the x-ray beam, were adhered to a glass microscope slide using double-sided tape and diffraction patterns were taken. Figure 29 a shows a selected portion of the pattern from the ribbon set with (baseline) composition $\left(\mathrm{Y}_{0.55} \mathrm{Nd}_{0.45}\right)_{2.2} \mathrm{Fe}_{14} \mathrm{~B}_{1.1}$. In this ribbon, strong $<00>$ texture was observed, consistent with previous results [59]. The $\{00\}$ peaks were greatly enhanced, while all other peaks were suppressed to such an extent that GSAS refinement could not be used effectively.

The Ag-containing alloys all had similar diffraction patterns to the one shown in Fig. 29b, i.e., a free side XRD pattern for ribbon with composition $\left(\mathrm{Y}_{0.55} \mathrm{Nd}_{0.45}\right)_{2.2} \mathrm{Fe}_{14} \mathrm{~B}_{1.1} \mathrm{Ag}_{0.15}$. This pattern shows a significant change in the texture of the ribbon, differing from both the isotropic case and the Ag-free baseline case. The (410) and (006) peaks (the peaks of greatest intensity in the isotropic and Ag-free cases, respectively) are both greatly suppressed, while the (214), (105), and, especially, (204) peaks are significantly enhanced. Thus, the aligned cells of the melt-spun alloys with $\mathrm{Ag}$ additions have a c-axis that is not perpendicular to the wheel surface, contrary to previous work ${ }^{4}$ on related alloys (and the baseline alloy) that were melt-spun at low wheel speed. The relative intensities of the (006), (410), and (204) for each diffraction scan of the Ag-containing alloys are listed in Table I, 
compared to the isotropic and baseline (Ag-free) cases. The unique, highly controlled pattern from Ag-modified alloys suggests that an alternate solidification path was followed, probably involving solidification catalysis by a Ag-containing phase. Several intermetallic compounds from Ag-RE [100, 102-104] and Ag-Fe-RE $[105,106]$ systems are candidates for such a catalyst. Detailed microstructural examination is in-progress to deduce the most likely phases involved and the solidification reaction sequence, but the previous findings suggest that epitaxial templating [59] is a likely mechanism.

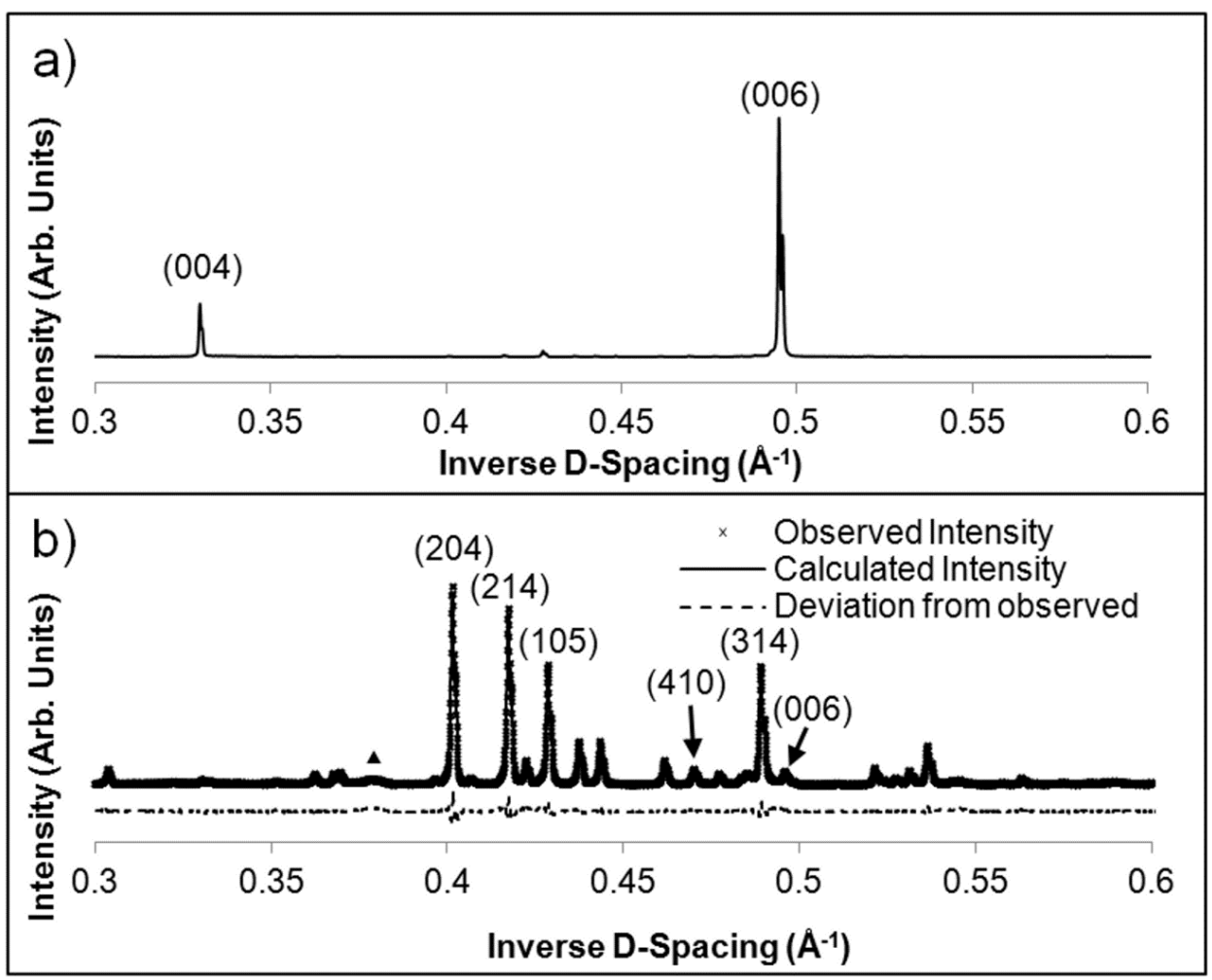

Figure 51 - Free side XRD patterns for melt-spun ribbon of composition a) $\left(\mathrm{Y}_{0.55} \mathrm{Nd}_{0.45}\right)_{2.2} \mathrm{Fe}_{14} \mathrm{~B}_{1.1}$ and $\left.b\right)\left(\mathrm{Y}_{0.55} \mathrm{Nd}_{0.45}\right)_{2.2} \mathrm{Fe}_{14} \mathrm{~B}_{1.1} \mathrm{Ag}_{0.15}$ with the accompanying Rietveld refinement trace. The triangle in $4 \mathrm{~b}$ marks the location again of the same peak (see Fig. 3) that corresponds to the AgRE (B2 structure) phase. 
Table 1 - Selected XRD peak intensities (for $\left.\left(\mathrm{Y}_{0.55} \mathrm{Nd}_{0.45}\right)_{2.2} \mathrm{Fe}_{14} \mathrm{~B}_{1.1} \mathrm{Ag}_{\mathrm{x}}\right)$ as a percentage of maximum intensity for each sample observed.

\begin{tabular}{|c|c|c|c|}
\hline Peak & $(006)$ & $(410)$ & $(204)$ \\
\hline Calculated Isotropic & $39.1 \%$ & $100.0 \%$ & $47.8 \%$ \\
\hline $\mathrm{x}=0.03 \mathrm{Ag}$ & $16.0 \%$ & $13.7 \%$ & $100.0 \%$ \\
\hline $\mathrm{x}=0.15 \mathrm{Ag}$ & $17.8 \%$ & $18.9 \%$ & $100.0 \%$ \\
\hline $\mathrm{x}=0.10 \mathrm{Ag}$ & $16.6 \%$ & $15.7 \%$ & $100.0 \%$ \\
\hline $\mathrm{x}=0.05 \mathrm{Ag}$ & $10.8 \%$ & $13.7 \%$ & $100.0 \%$ \\
\hline $0 \mathrm{Ag}$ (Baseline) & $100.0 \%$ & $0.9 \%$ & $0.8 \%$ \\
\hline
\end{tabular}

\section{Conclusions and Implications}

The addition of $\mathrm{Ag}$ to RE-Fe-B alloys provided significant modification to the microstructure and growth observed. A columnar structure was stabilized through nearly the entire thickness of the ribbon. The spacing of these columns increased gradually as growth proceeded and this trend seemed to vary slightly with $\mathrm{Ag}$ concentration. The $\mathrm{Ag}$ also altered the texture in the ribbons, indicating a disruption in the normal $\mathrm{RE}_{2} \mathrm{Fe}_{14} \mathrm{~B} / \alpha-\mathrm{Fe}$ templating mechanism [59] for Ag-free magnet alloy ribbons melt-spun at low wheel speeds. While the novel texture obtained is not a single crystalline orientation (as desired for ease in optimum alignment), understanding of the new solidification sequence could lead to additional refinement and more complete, through-thickness alignment. Such improved ribbon with a highly dominant crystalline alignment may be utilized to produce crushed fragments for bonded magnet fabrication procedures (possibly involving low melting metallic matrix materials).

\section{Acknowledgements}

This work was supported by the US Department of Energy-Office of Energy Efficiency and Renewable Energy, Vehicle Technology Office, USDRIVE, and the PEEM program, under Contract No. DE-AC02-07CH11358 for the operation of Ames Laboratory (USDOE). 


\title{
Chapter 7 - Alteration of the solidification microstructure and texture of melt-spun RE-Fe-B alloys by the addition of $\mathrm{Ag}$
}

A manuscript to be submitted to Acta Materialia

N.T. Oster, M.J. Kramer, K.W. Dennis, I.E. Anderson, and R.W. McCallum

\begin{abstract}
Control of texture and microstructure is vital to improving magnetic properties of $\mathrm{RE}_{2} \mathrm{Fe}_{14} \mathrm{~B}$-type permanent magnet materials. This study documents the effects of small (0.86 at.\%) Ag additions on the texture and microstructure of meltspun $\left(\mathrm{Y}_{0.55} \mathrm{Nd}_{0.45}\right)_{2.2} \mathrm{Fe}_{14} \mathrm{~B}_{1.1}$. At low wheel speeds, a columnar dendritic structure was observed and precipitates rich in $\mathrm{Ag}$ and $\mathrm{Nd}$ lined the column boundaries. Transmission electron microscopy (TEM) indicated that fine precipitates also are within the matrix with an average size of approximately $10 \mathrm{~nm}$. Local electrode atom probe (LEAP) revealed that the precipitates primarily consist of $\mathrm{Nd}$ and $\mathrm{Ag}$ in approximately a 1:1 ratio. Texture of the ribbon "free-side" surfaces was observed using x-ray diffraction (XRD). In ribbons of similar composition (without $\mathrm{Ag}$ ) the basal plane was observed parallel to the ribbon surface despite the a-axis easy growth direction, consistent with typical results that template on transient Fe dendrites. With the $\mathrm{Ag}$ addition, $\mathrm{x}$-ray diffraction patterns indicated that the ribbons melt-spun at low wheel speed $(5-10 \mathrm{~m} / \mathrm{s})$ are textured such that the basal plane is neither perpendicular to nor parallel to the plane of the ribbon. As the wheel speed is increased, the degree of texture is decreased. The authors propose that the unusual texture is induced through a modification of the solidification pathway caused by the presence of $\mathrm{Ag}$. The Ag apparently promotes a unique solidification mechanism that triggers transient primary dendrites of an unknown phase displaying 3-fold symmetry and a composition that decomposes rapidly to $\mathrm{RE}_{2} \mathrm{Fe}_{14} \mathrm{~B}$.
\end{abstract}

\section{Introduction}

RE-Fe-B alloys are typically used where high performance permanent magnets are required. (e.g. wind turbine electric generators or traction motors for hybrid or electric vehicles.) The alloys are based around the $\mathrm{Nd}_{2} \mathrm{Fe}_{14} \mathrm{~B}$ structure [11, 
12], which displays the highest observed energy product to date. It has been proposed that polymer-bonded magnets might be used in some of these applications due to processing advantages (e.g. reduced corrosion and the ability to injection mold the material directly into a rotor cavity). However, in order to obtain the greatest properties out of these materials, anisotropic structures must be formed. Current commercial particulates for use in anisotropic bonded magnets (i.e. die upset [44] and HDDR [32] powders) suffer from a few factors which make them unappealing alternatives to their sintered magnet counterparts, including reduced temperature stability [36] and increased processing costs. A simplified method of production would be desirable.

Isotropic particulate, on the other hand, has been suitably fabricated through rapid solidification methods (both melt-spinning [107] and gas atomization [4]). Typical isotropic particulate will be "over-quenched" to form glassy material, followed by annealing to fully crystallize the structure and maintain small grain sizes. In order to achieve the "over-quenched" state, wheel speeds in excess of $20 \mathrm{~m} / \mathrm{s}$ are typically used. At lower wheel speeds $(5-15 \mathrm{~m} / \mathrm{s})$, however, textured material is often observed.

Single crystal experiments by Tenaud et al. [99] indicated that rapid growth in $\mathrm{Nd}_{2} \mathrm{Fe}_{14} \mathrm{~B}$ crystals occurred in the basal plane of the tetragonal structure. Based on this, one would expect the $c$-axis to be parallel to the wheel surface in the melt-spun ribbon. However, the low wheel speed ribbon typically displays a texture in which the c-axis is perpendicular to the wheel surface. This was explained by Kramer et al. [59] in 2002. The phase diagram for the Nd-Fe-B system [26] indicates that the $\mathrm{Nd}_{2} \mathrm{Fe}_{14} \mathrm{~B}$ structure lies below a $\mathrm{Fe}+\mathrm{L}$ phase field. As a result, $\mathrm{Fe}$ dendrites grow from the wheel surface toward the free surface in their $<100>$ directions. As the alloy cools through the peritectic reaction, $\mathrm{Nd}_{2} \mathrm{Fe}_{14} \mathrm{~B}$ epitaxially templates to the primary Fe via the relationship [110](110) $)_{\alpha-F e} \|[110](006)_{2-14-1}[59]$ and grows in a basal plane direction. The result is a columnar structure that displays a texture in which the basal plane is parallel to the ribbon surface. While the texture is desirable, the grain structure is typically too coarse to maintain significant coercivity. If, 
however, the grain structure could be refined, while still maintaining the texture, a suitable coercivity in an anisotropic structure could be obtained.

Previously, the authors proposed that the use of a segregating additive (such as Ag) might be able to refine the grain structure [108]. Ag was added to the alloy in various concentrations and the effects on microstructure were observed. One notable finding was that, when Ag was added, the texture observed was altered from a c-axis texture (when no Ag was present) to a texture where the c-axis was neither parallel nor perpendicular to the surface. This indicates a clear alteration of the mechanism of texture formation. In this work, further characterization of the phenomenon is completed and a possible mechanism is discussed.

\section{Experimental procedure}

Ingots of composition $\left(\mathrm{Y}_{0.55} \mathrm{Nd}_{0.45}\right)_{2.2} \mathrm{Fe}_{14} \mathrm{~B}_{1.1} \mathrm{Ag}_{0.15}$ were arc melted on a water-cooled copper hearth using a non-consumable tungsten electrode. The ingots were then placed in a quartz crucible with a $0.8 \mathrm{~mm}$ circular orifice, inductively heated to $1400^{\circ} \mathrm{C}$, and free jet melt-spun on a rotating copper wheel. An atmosphere of $1 / 3 \mathrm{~atm}$. high purity He was maintained in the chamber and an additional $1 / 6 \mathrm{~atm}$. He was used to eject the melt. Wheel speed was varied from $5-20 \mathrm{~m} / \mathrm{s}$.

The resulting ribbon was then characterized using scanning electron microscopy (SEM) with energy dispersive spectroscopy (EDS), differential scanning calorimetry (DSC), x-ray diffraction (XRD), transmission electron microscopy (TEM), and local electrode atom probe (LEAP).

\section{Results}

The ribbons were mounted as transverse cross-sections, perpendicular to the melt spinning direction, in epoxy, were ground and polished, and were observed in a JEOL JSM 5910LV SEM. Representative backscattered electron (BSE) images of ribbon melt-spun at $5 \mathrm{~m} / \mathrm{s}$ and $10 \mathrm{~m} / \mathrm{s}$ can be seen below in a and $b$, respectively. At the bottom of the images is the portion of ribbon that was in contact with the chill wheel. A region of fine, equiaxed grains can be observed at the wheel surface of the ribbon. Growing out of these fine grains, a columnar structure extends through the 
thickness of the ribbon. Dispersed along the length of the cells is a fine, Ag-rich second phase. The "bead-like" morphology of the phase indicates that it likely coarsened upon cooling. This second phase, however, is too fine to be accurately quantified using energy dispersive spectroscopy (EDS) in the SEM. EDS analysis of similar product phases was conducted in the SEM by cross-sectioning one of the parent ingots. The analysis of the bright phase indicated an average composition of $46 \mathrm{Ag}-44 \mathrm{Nd}-5 \mathrm{Y}-5 \mathrm{Fe}$ (at. \%). This indicates that the second phase is likely $\mathrm{AgNd}$ (with some $\mathrm{Y}$ possibly substituted for $\mathrm{Nd}$ ), consistent with the observations of Takeda et al. [105] in their study of $\mathrm{Nd}-\mathrm{Fe}-\mathrm{Ag}$ phase equilibrium.
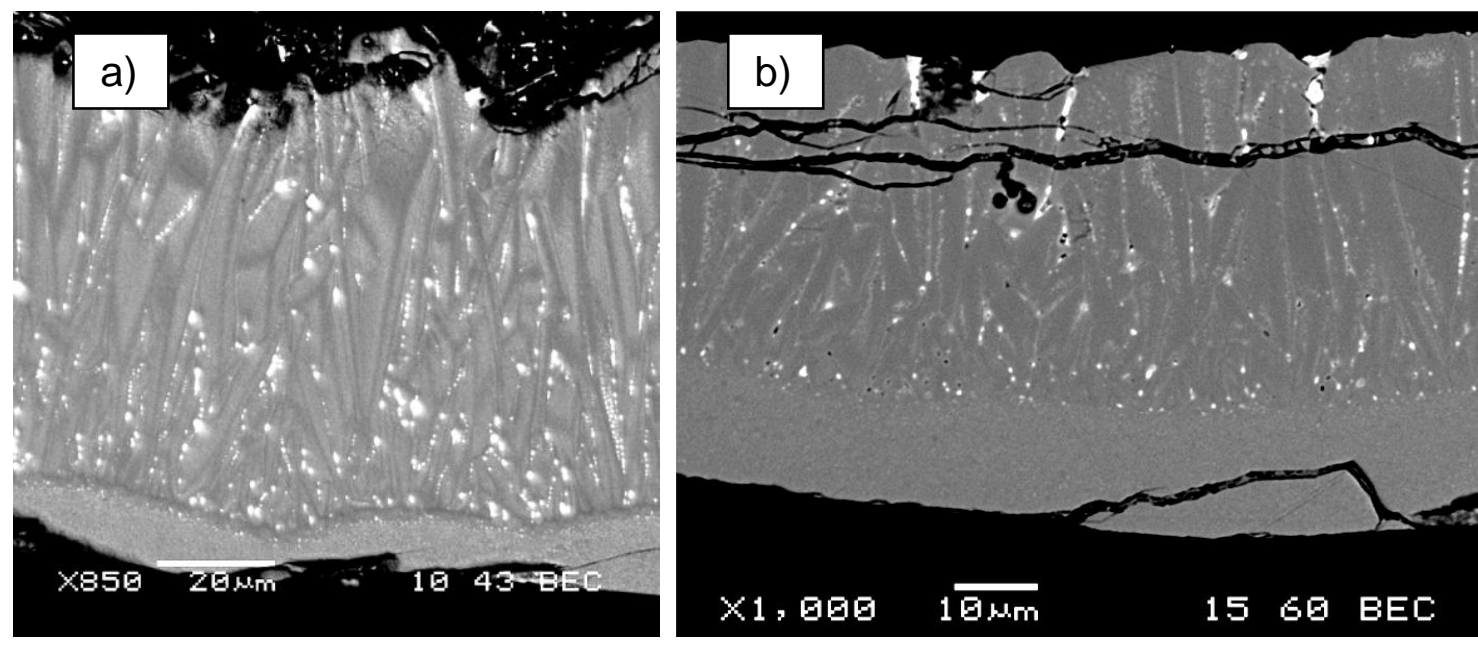

Figure 52 - Cross section backscattered electron micrograph of $\left(Y_{0.55} \mathrm{Nd}_{0.45}\right)_{2.2} \mathrm{Fe}_{14} B_{1.1} \mathrm{Ag}_{0.15}$ melt-spun at a) $5 \mathrm{~m} / \mathrm{s}$ and b) $10 \mathrm{~m} / \mathrm{s}$. The bottom of the image represents the ribbon wheel surface.

One of the ribbons that was melt-spun at $5 \mathrm{~m} / \mathrm{s}$ was mounted and polished from the free surface in order to observe the columns end-on. The resulting BSE image can be seen in Figure 53. The dark contrast structure appears to be dendrite cores which form in a "star-shaped" morphology with 3-fold symmetry. The points of the "stars" appear to grow until impinging on a neighboring column. In the space between the arms of these dendrite cores, several of the (bright contrast) Ag-rich precipitates are seen. 


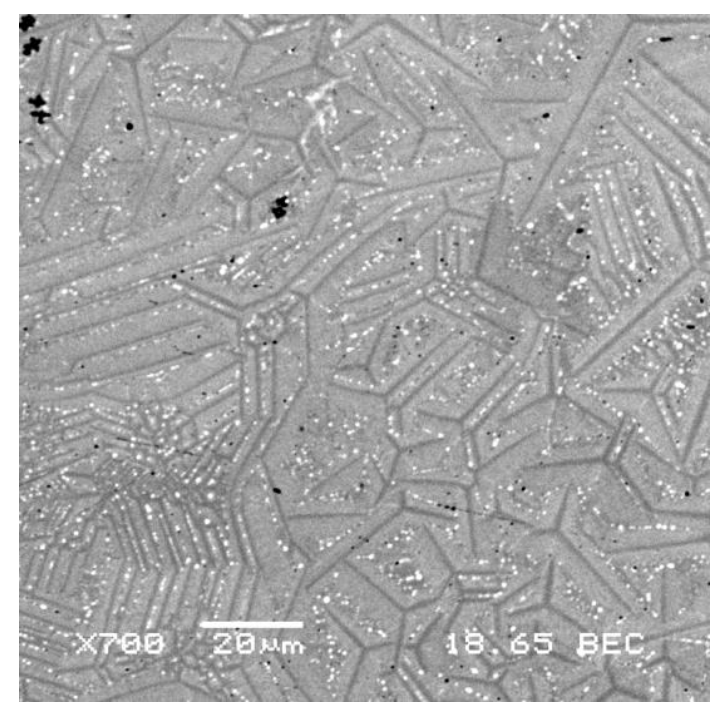

Figure 53 - BSE micrograph of $\left(\mathrm{Y}_{0.55} \mathrm{Nd}_{0.45}\right)_{2.2} \mathrm{Fe}_{14} \mathrm{~B}_{1.1} \mathrm{Ag}_{0.15}$ melt-spun at $5 \mathrm{~m} / \mathrm{s}$ taken parallel to the plane of the ribbon near the free surface.

X-ray diffraction was conducted on a sample of the ribbon that was meltspun at $5 \mathrm{~m} / \mathrm{s}$, crushed to powder, and scanned using a Panalytical X'Pert PRO diffractometer with Co K- $\alpha$ radiation. Scans were taken from $20-130^{\circ} 2 \theta$ at $0.008^{\circ}$ intervals. A Rietveld refinement confirmed the phase structures present using General Structure Analysis System (GSAS) software [101, 109]. Figure 54 shows a selected range of the $\mathrm{x}$-ray diffraction trace observed and the trace calculated by the refinement. Calculated peak locations for $\mathrm{RE}_{2} \mathrm{Fe}_{14} \mathrm{~B}$ match well with those observed, with the exception of one peak, located at approximately $39.7^{\circ} 2 \theta$. The peak is very wide, of low intensity and is likely contributed by the Ag-containing intermetallic phase since it corresponds with the location of the greatest intensity peak in the AgRE compound. However, a single peak is not enough to definitively identify the phase. A piece of the parent ingot was also crushed and $x$-rayed. The resulting pattern was nearly identical to that of the crushed ribbon. 


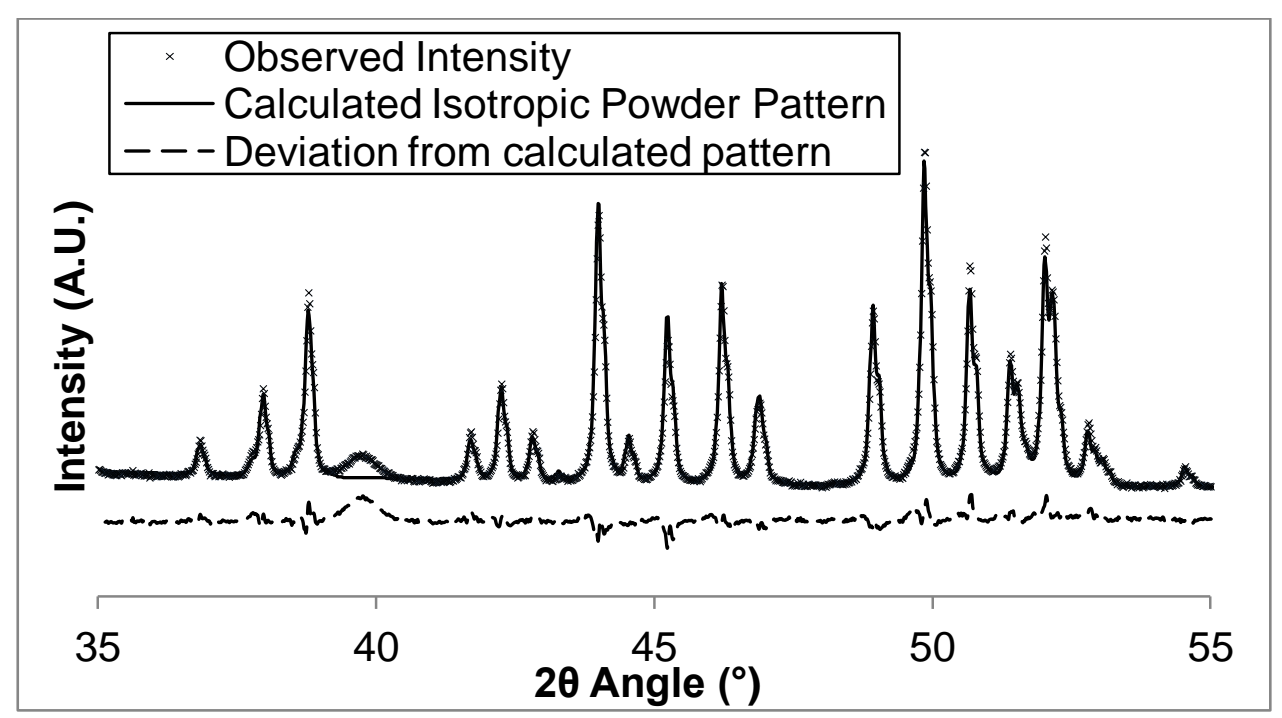

Figure 54 - X-ray diffraction Trace of crushed $\left(\mathrm{Y}_{0.55} \mathrm{Nd}_{0.45}\right)_{2.2} \mathrm{Fe}_{14} \mathrm{~B}_{1.1} \mathrm{Ag}_{0.15}$ ribbon meltspun at $5 \mathrm{~m} / \mathrm{s}$. Also pictured, Rietveld refinement data.

To analyze the extended columnar texture, approximately 10 ribbon pieces from each wheel speed were adhered to a glass slide using double-sided tape with the free surface facing up. Figure 55 shows the diffraction patterns observed for each wheel speed.

It can be seen that, while peak locations match well with the calculated values for $\mathrm{RE}_{2} \mathrm{Fe}_{14} \mathrm{~B}$, the strongest degree of texturing is present in the silvercontaining alloy at lower wheel speeds. As wheel speed is increased, the degree of texture within Ag-containing ribbons is decreased, with the $15 \mathrm{~m} / \mathrm{s}$ ribbon exhibiting very little texture. At the $5 \mathrm{~m} / \mathrm{s}$ wheel speed, the (204) and (214) peaks are greatly enhanced, while the (105) and (314) peaks display little change in relative intensity. Since the greatly enhanced peaks contain a strong c-axis component, it is apparent that the c-axis is not parallel to the surface of the ribbon. Many other peaks are suppressed. Particularly notable are the significantly suppressed (410) and (006) peaks, which are the peaks of greatest intensity in both the isotropic pattern and in the c-axis textured patterns typically observed in low wheel speed ribbons without Ag additions. 


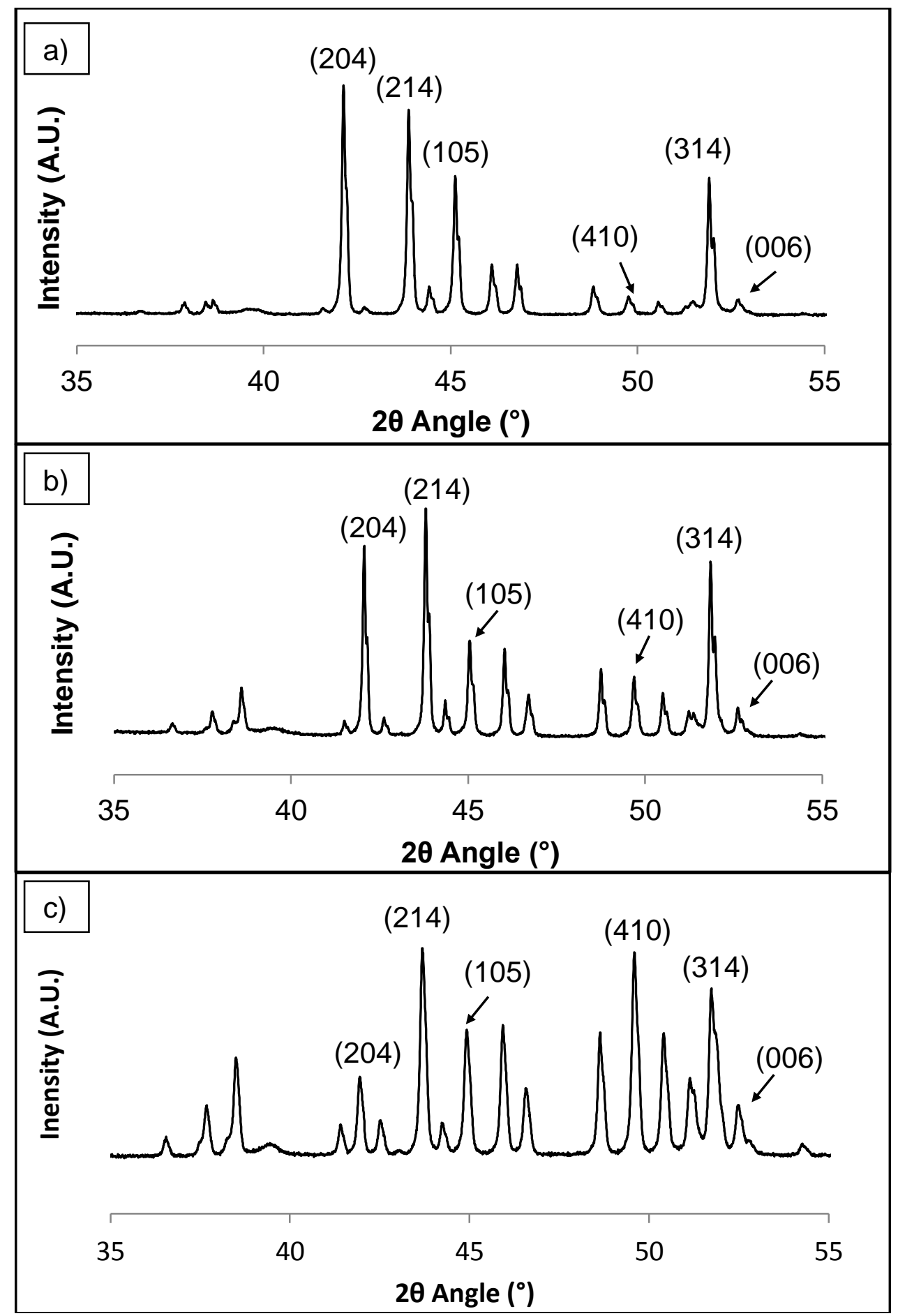

Figure 55 - Free surface $x$-ray diffraction plot for ribbons of composition $\left(\mathrm{Y}_{0.55} \mathrm{Nd}_{0.45}\right)_{2.2} \mathrm{Fe}_{14} \mathrm{~B}_{1.1} \mathrm{Ag}_{0.15}$ melt-spun at (a) $5 \mathrm{~m} / \mathrm{s}$, (b) $10 \mathrm{~m} / \mathrm{s}$, and (c) $15 \mathrm{~m} / \mathrm{s}$.

Differential scanning calorimetry (DSC) was done on each of the samples to observe thermal events. Approximately $45 \mathrm{mg}$ of ribbon was placed in an $\mathrm{Al}_{2} \mathrm{O}_{3}$ 
crucible. The crucible was heated at $20^{\circ} \mathrm{C} /$ minute under flowing high purity He. A portion of the resulting thermal trace can be seen in Figure 56 . An event at $300^{\circ} \mathrm{C}$ corresponds to the Curie temperature of the alloy. At $948^{\circ} \mathrm{C}$ a second thermal event is observed, corresponding closely to the melting temperature of the $\mathrm{AgNd}$ phase $\left(952^{\circ} \mathrm{C}\right)$. Upon close observation, very small thermal events can be seen near $750^{\circ} \mathrm{C}$ and $650^{\circ} \mathrm{C}$. Comparing these temperatures to the $\mathrm{Ag}-\mathrm{Nd}$ phase diagram, the events occur near the allotropic phase transformations in the $\mathrm{Ag}_{2} \mathrm{Nd}$ compound (at $635^{\circ} \mathrm{C}$ and $780^{\circ} \mathrm{C}$ ). While only one trace is shown, it should be noted that no significant differences between the traces from the three different batches of ribbons were observed.

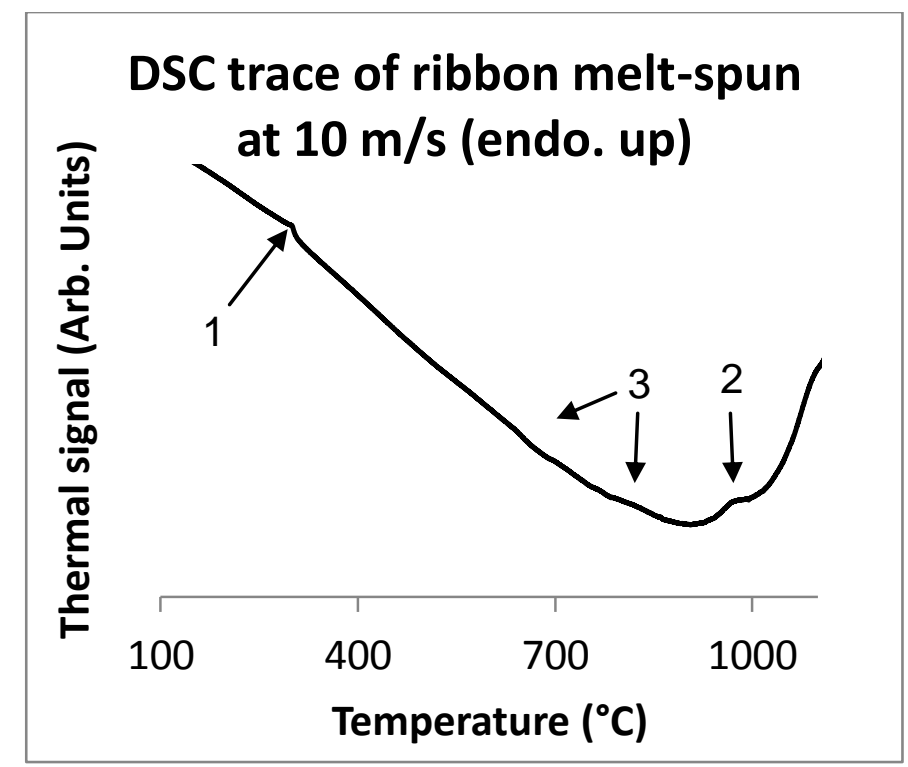

Figure 56 - DSC trace for $\left(\mathrm{Y}_{0.55} \mathrm{Nd}_{0.45}\right)_{2.2} \mathrm{Fe}_{14} \mathrm{~B}_{1.1} \mathrm{Ag}_{0.15}$ ribbon melt-spun at $10 \mathrm{~m} / \mathrm{s}$. The arrow marked "1" points to the Curie temperature. The arrow marked "2" points to the melting temperature of the AgNd phase. Arrows labeled "3" show points of small thermal events in the trace, closely corresponding to the allotropic phase transition temperatures in the $\mathrm{Ag}_{2} \mathrm{Nd}$ structure.

In order to characterize the precipitate morphology and distribution, both cross section (foil 1) and in-plane (foil 2) TEM foils were made from the ribbon meltspun at $5 \mathrm{~m} / \mathrm{s}$. A FEI Quanta 3D FEG focused ion beam (FIB) was used to create the foils with electron-transparent regions near the interface between fine equiaxed 
grains and the columnar grains (exact locations can be seen in Figure 57). A Tecnai $G^{2}$ F20 TEM and Phillips CM30 were used to image the foils. Figure 58 is a composite of TEM bright field images showing nearly the entire electron transparent region in the foil. At the bottom of the image, the fine equiaxed region can be seen. In the center of the image the sharp transition to a columnar grain structure is seen. The image shows that some interfaces have a secondary phase in the intergranular region, while others do not. Figure 59a shows a bright field TEM image taken from the columnar region. Along the grain boundaries, the "bead-like" precipitates can be seen coating the grain boundaries. EDS measurements indicate that the composition of the phase indicated by arrow "1" is approximately 67Ag-29Nd-4Y (in at. \%). This implies that the grain boundary precipitates are $\mathrm{Ag}_{2} \mathrm{Nd}$ with a small portion of the Nd replaced by Y. Arrow "2" shows a phase of composition 76Fe-18Y$6 \mathrm{Nd}$ (in at. \%). The arrow labeled "3" showed a phase with composition 87Fe-10Y$3 \mathrm{Nd}$ (in at. \%), very close to the composition of the $\mathrm{RE}_{2} \mathrm{Fe}_{14} \mathrm{~B}$ structure. The phase assemblage in the triple junctions between grains is also rather complex, showing at least 3 separate phases (see Figure 59b). EDS measurements indicate the composition of these phases to be $50 \mathrm{Ag}-44 \mathrm{Nd}-6 \mathrm{Y}$ at.\% (arrow "5"), 93Nd-6Y-1Fe at.\% (arrow "6"), and 72Fe-15Y-12Nd at.\% (arrow "7").

In the interior of the grains, fine precipitates are also observed. These are seen to have a size range of about 2-50 $\mathrm{nm}$, with the majority of the precipitates around $7 \mathrm{~nm}$. In the larger precipitates it is apparent that the morphology is disk-like. However, there is no directly observed indication of coherence between the precipitates and the matrix. Near some of the grain boundaries, a region depleted in these precipitates is seen. Regions in the center of the grains also show depletion of the precipitates. Close observation (Figure 63) shows that the regions are "starshaped" like what was seen in Figure 53. It can also be seen that the precipitates form lines extending from the arms of the "stars", a possible indication of dendrite arms. These lines show average spacings of $\sim 80 \mathrm{~nm}$. 


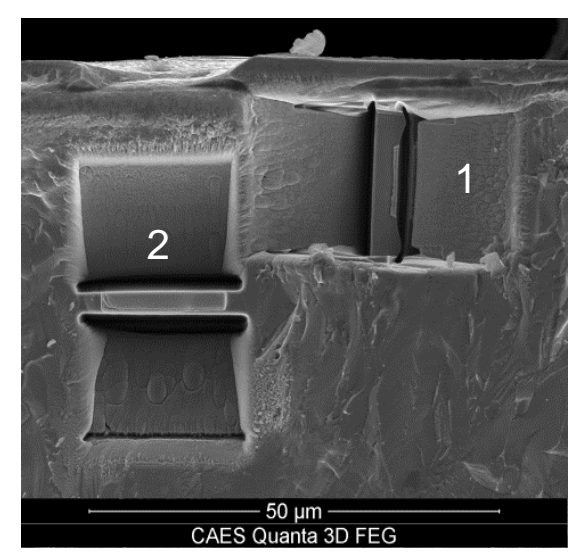

Figure 57 - Locations of TEM foils on ribbon cross section. The top of the image is the surface of the ribbon that was in contact with the wheel. The foil marked " 1 " spanned across the interface between equiaxed and columnar grains. The foil marked "2" was an in-plane section in the columnar region.

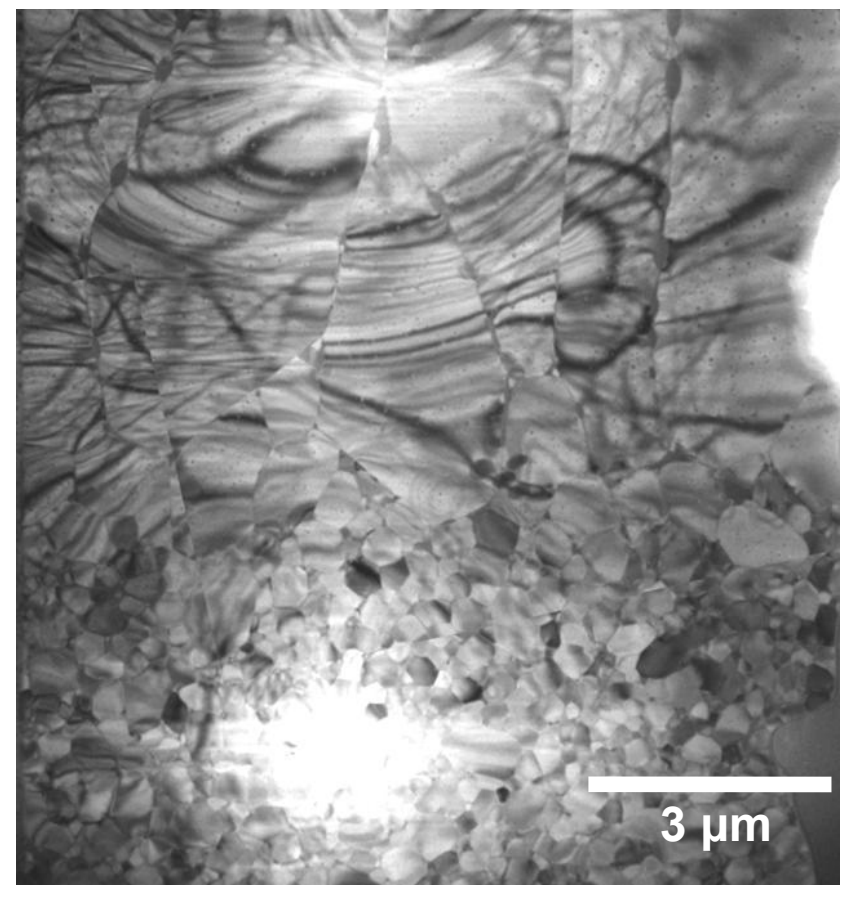

Figure 58 - A composite of brightfield TEM images showing virtually the entire electron transparent region in the cross section TEM foil (foil 1). 

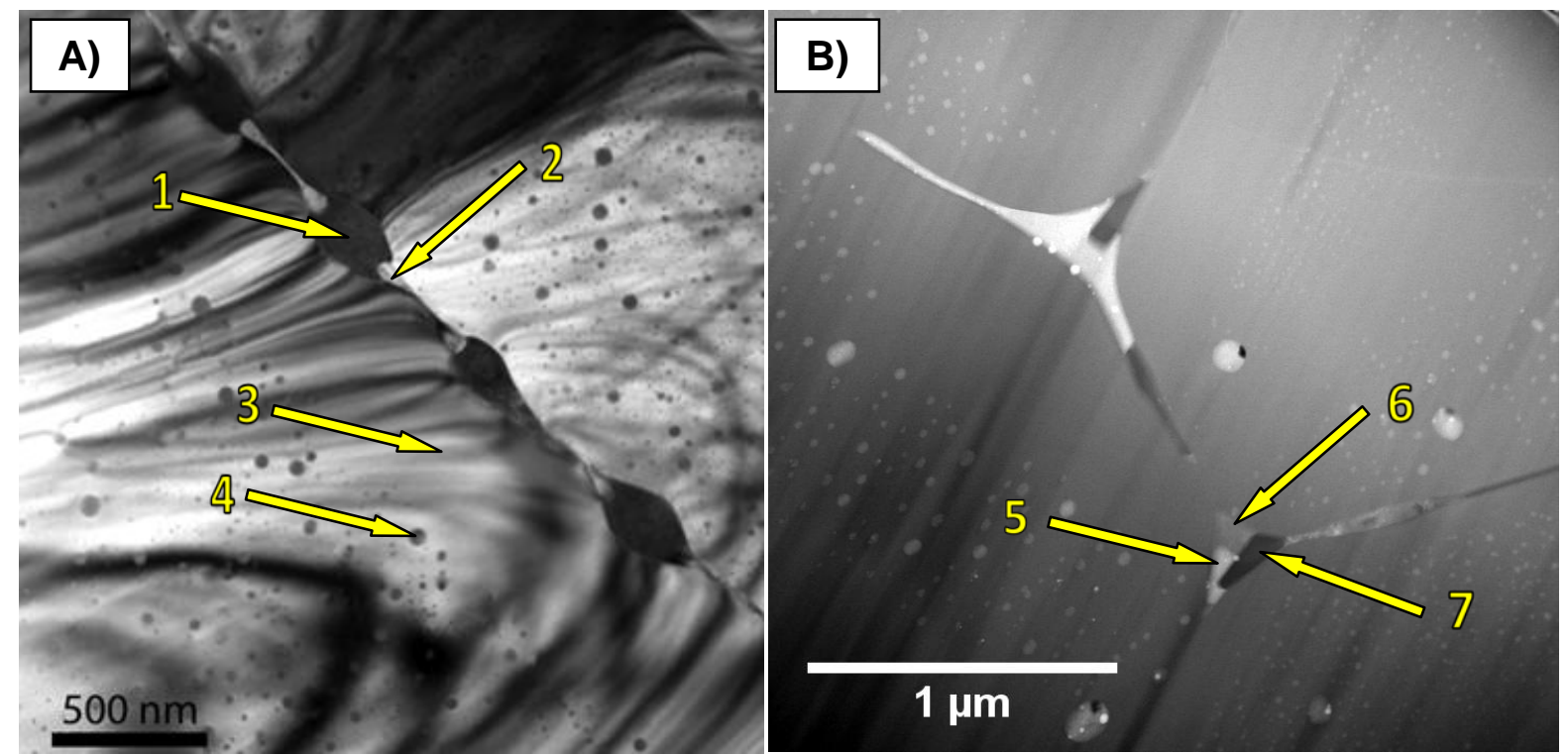

Figure 59 - A) Bright field TEM image of columnar region in foil 1. The arrow labeled " 1 " points to the grain boundary precipitates. A second phase at the grain boundaries is indicated by arrow " 2 ". The arrow labeled " 3 " points to the region depleted in precipitates. The arrow labeled "4" points to the fine precipitates located within the matrix. B) In-plane high angle annular dark field (HAADF) STEM image of the grain boundaries in foil 2. Arrows 5-7 point to three different phases observed within the grain triple junctions.

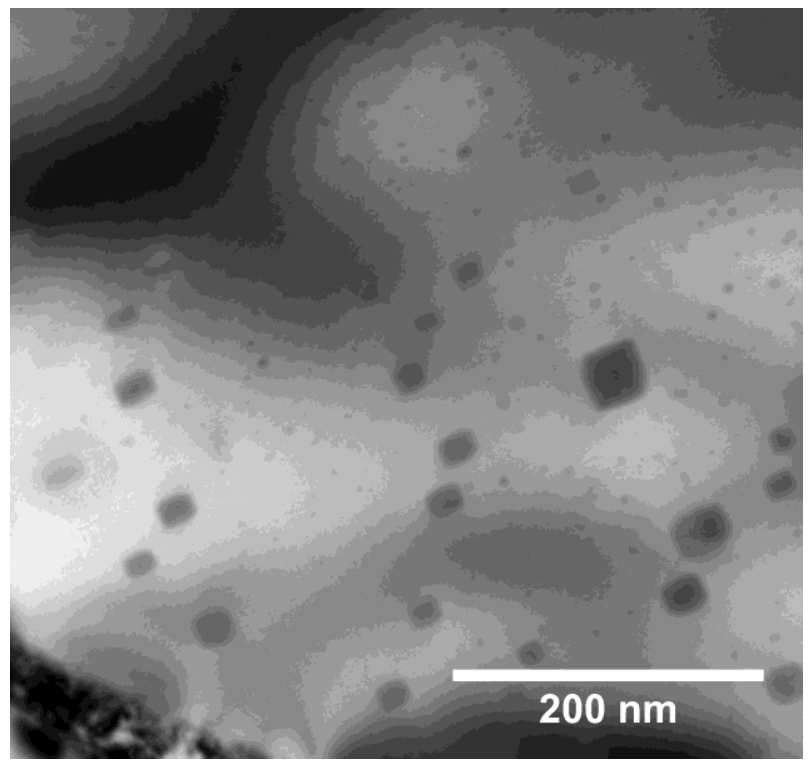

Figure 60 - Bright field TEM image of the fine precipitates seen in foil 2. The largest of these precipitates display some degree of faceting and appear to have an orientation relationship with the matrix. 


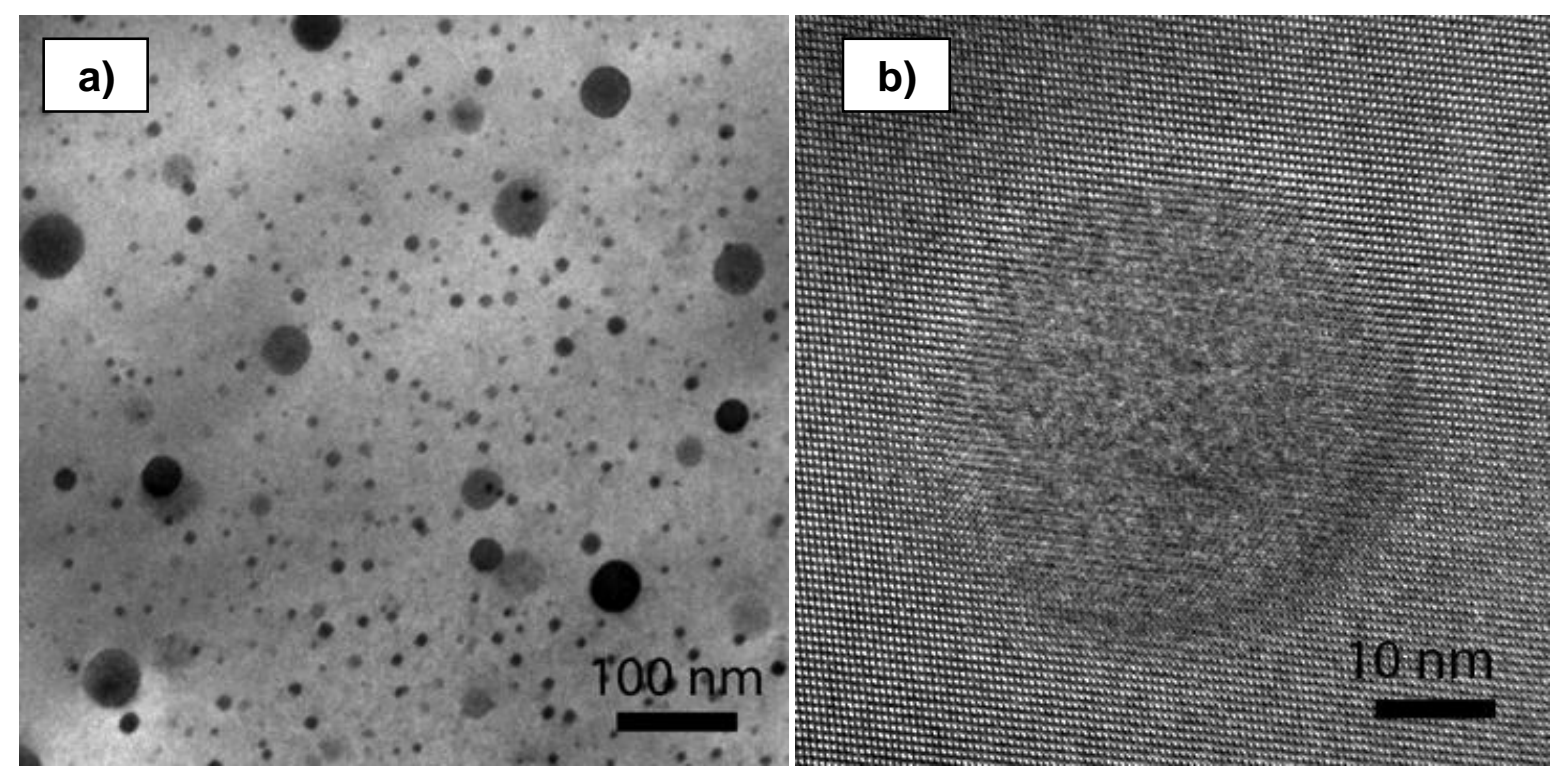

Figure 61 - Bright field TEM images of fine precipitates from foil 1. The distribution and size of the precipitates can be seen in image a). A high resolution image of one of the larger precipitates is seen in image b).

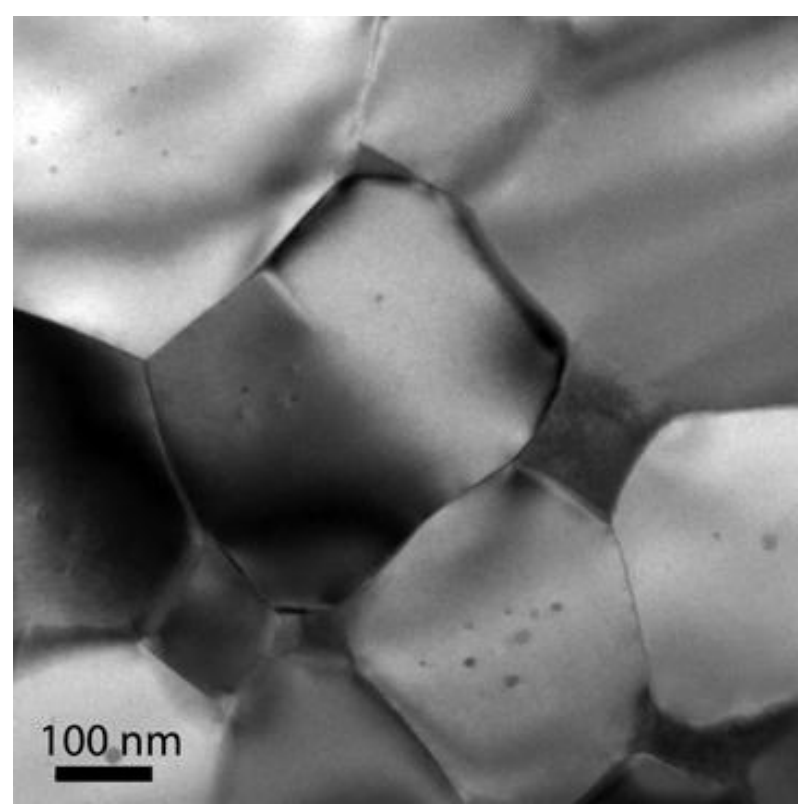

Figure 62 - Bright field TEM image of the fine, equiaxed region in foil 1. Fine precipitates can be seen near the center of the grains. The phase enriched in Ag and $\mathrm{Nd}$ is also observed at grain triple junctions. 


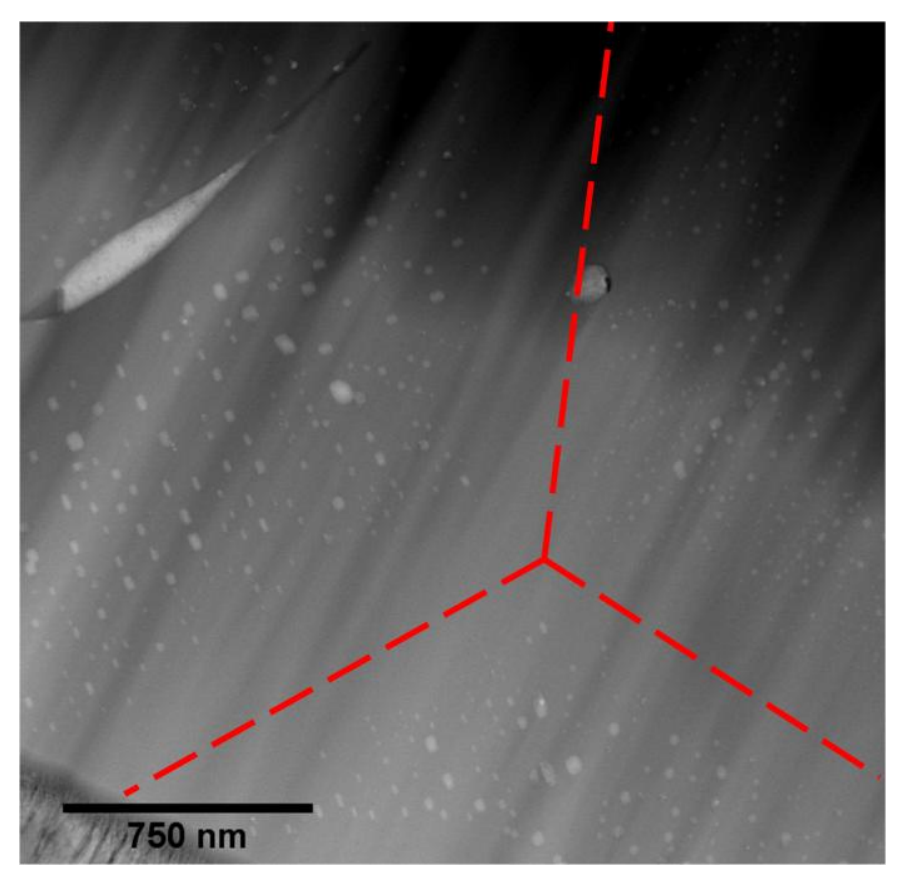

Figure 63 - In-plane HAADF STEM image of foil 2. The drawn-in dashed lines show regions in the center of the grain where the fine precipitates are not found. The pattern is similar to what is shown in the SEM image in Figure 53. Note that other regions free of precipitates can be seen at the edge of the grain near bordering second phases.

To further characterize the precipitates, the FIB was used to prepare atom probe tips of the alloy for LEAP tomography. The tips were fabricated to have a tip radius of $\sim 40 \mathrm{~nm}$. A Cameca LEAP 4000X HR was then used in voltage mode and approximately 40 million data points were collected. Figure 64 shows elemental maps taken from the tip reconstruction. It can be seen that the precipitates are enriched in $\mathrm{Nd}, \mathrm{Ag}, \mathrm{C}$, and $\mathrm{H}$. Chemical analysis of the LEAP data indicates that the precipitates are primarily $\mathrm{Nd}$ and $\mathrm{Ag}$, and that the $\mathrm{Nd}: \mathrm{Ag}$ ratio is approximately $1: 1$ within the precipitates. 

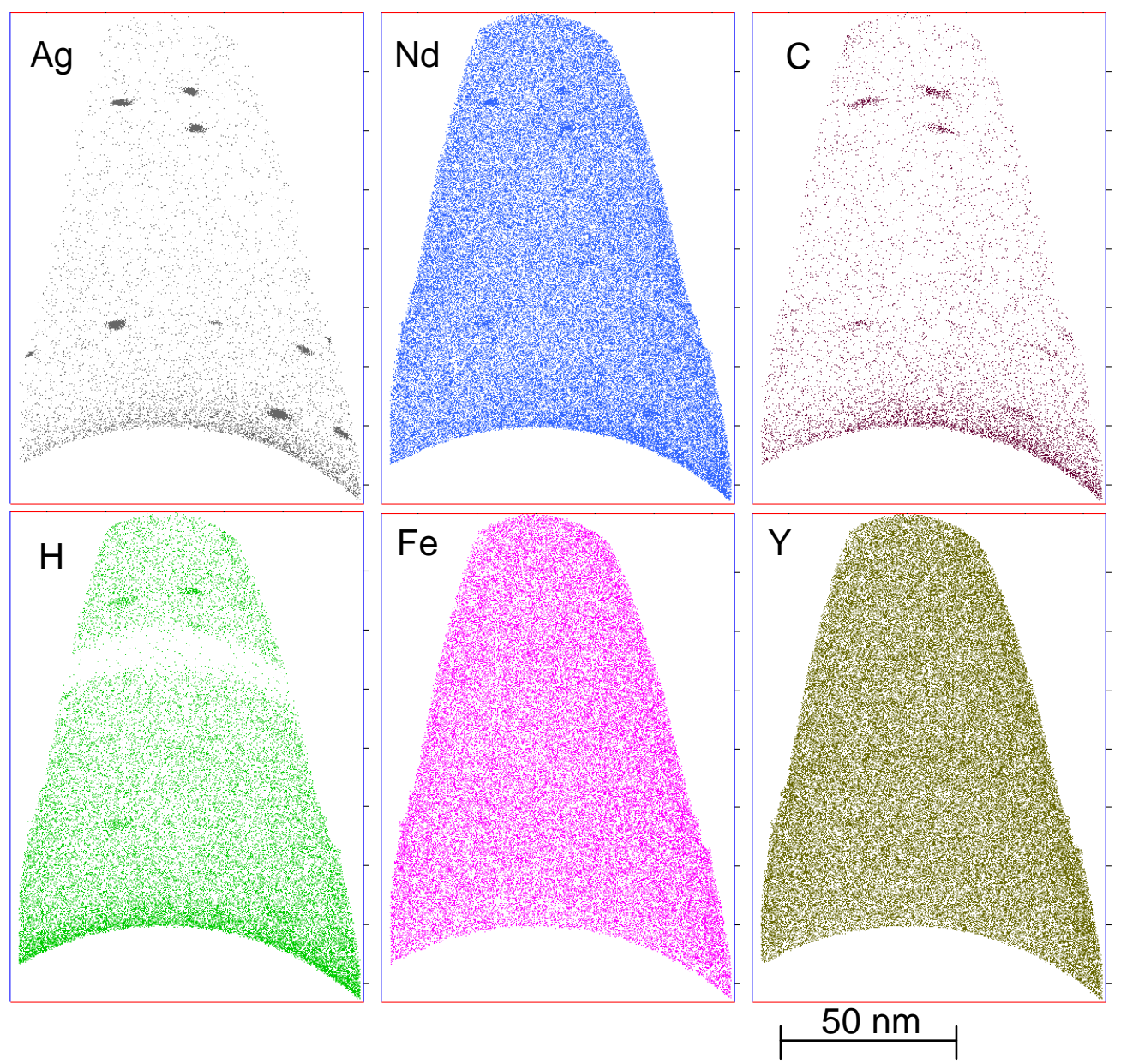

Figure 64 - Elemental maps taken from the LEAP data reconstruction. The maps indicate that the precipitates are enriched in $\mathrm{Ag}, \mathrm{Nd}, \mathrm{C}$, and $\mathrm{H}$.

\section{Discussion}

\section{Precipitates}

The formation of precipitates within the matrix of the alloy, as well as the morphology of the precipitates along the grain boundaries is unusual for $\mathrm{RE}_{2} \mathrm{Fe}_{14} \mathrm{~B}$ alloys. Typically, most elements that are insoluble in the matrix are pushed to the intergranular regions. These then segregate to the triple junctions and/or form a thin film along the grain boundaries. In the alloy observed here, much of the silver and excess neodymium is pushed to triple points and grain boundaries, while some exists in the form of fine precipitates within the matrix. The "bead-like" structure of 
the grain boundary precipitates indicates that coarsening of the structure likely occurred. This could be either a result of high interfacial free energy between the matrix and the precipitates, or that the precipitates were still in the liquid form when pushed to the grain boundaries and began to coalesce prior to solidification. The presence of several phases at the grain boundaries and in triple junctions may be indicative of segregation within the liquid. As the liquid becomes enriched with rejected $\mathrm{Ag}$, two liquid phases may become stable, as miscibility gaps are seen in both the Ag-Fe [110] and Ag-B systems [100].

The larger of the fine precipitates trapped within the matrix show a disklike morphology. Within one dendrite arm, they are all similarly oriented. It is likely that liquid pockets are trapped between tertiary dendrite arms and the precipitates are subsequently formed [111]. Another possibility is that the matrix is supersaturated with $\mathrm{Ag}$ at high temperature and subsequent precipitation occurs upon cooling [112]. However, this explanation does not fully account for the lines of precipitates that are seen in Figure 63.

Figure 65 shows the particle size distribution calculated from the image in Figure 61a. Precipitate size and morphology like what is observed here is not entirely unprecedented. Work on $\mathrm{Nb}$ additions to $\mathrm{Nd}-\mathrm{Fe}-\mathrm{B}$ alloys resulted in a similar distribution of similarly sized precipitates dispersed throughout the matrix [113-117]. The precipitates reported were hexagonal ternary $\mathrm{Nb}-\mathrm{Fe}-\mathrm{B}$ compounds that were identified as being coherent with the matrix. It was observed that coercivity was slightly enhanced with the presence of these precipitates $[118,119]$, and was surmised that the precipitates acted as magnetic domain wall pinning sites. Lorentz force microscopy indicated that the domain walls did, indeed, interact with the precipitates. It is possible that these Ag-Nd precipitates could serve a similar function, possibly even allowing for larger grain sizes while maintaining satisfactory coercivity. 


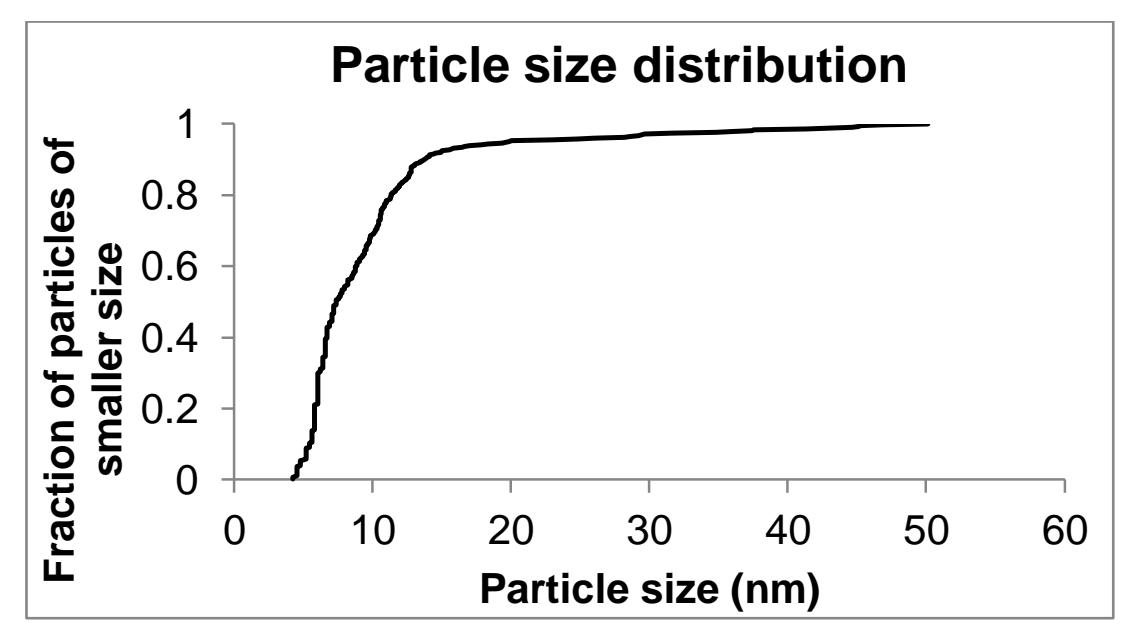

Figure 65 - Precipitate size distribution

LEAP tomography indicates that the precipitates in the matrix are enriched in $\mathrm{Nd}$ and $\mathrm{Ag}$ in an approximately 1:1 ratio. In the Ag-Nd phase diagram [100], several stable intermetallics have been identified [102-104], including the AgNd, $\mathrm{Ag}_{2} \mathrm{Nd}$, and $\mathrm{Ag}_{51} \mathrm{Nd}_{14}$ phases. This indicates a high likelihood that the fine precipitates consist primarily of the 1:1 intermetallic compound.

\section{Texture}

In this study, the effect of wheel speed on texture was also observed. The degree of texture was seen to decrease as wheel speed increased. This is consistent with the mechanism proposed by Kramer for traditional RE-Fe-B alloys. In this mechanism, as wheel speed is increased the solidification front velocity of the melt is increased to a speed rapid enough to disrupt the formation of columnar dendrites through the thickness of the ribbon. Without the oriented primary dendrites, no oriented template is formed for the creation of textured $\mathrm{RE}_{2} \mathrm{Fe}_{14} \mathrm{~B}$.

One finding of particular interest was an alteration in the observed texture in low wheel speed ribbons from the texture expected in alloys with no Ag added, indicating a change in the mechanism. The (204), (214), and (105) peaks are among those enhanced in the unusual pattern.

It should be noted that the enhanced peaks correspond closely to the high intensity peaks observed in the $\mathrm{Ag}_{51} R E_{14}$ structure. This could potentially indicate an 
epitaxial relationship between the 2-14-1 structure and the 51-14 structure due to similar planar spacings between the two crystal structures. The phase could form from solute rejected from the equiaxed zone and subsequently provide a seed crystal for the oriented columns. However, the $A g_{51} R E_{14}$ structure is not observed in the refined XRD patterns or readily identified within the TEM micrographs. It is possible that the phase could be transient and is consumed upon recalescence or as the Ag-rich phase cools through the peritectic reaction $\left(\mathrm{Ag}_{51} \mathrm{Nd}_{14}+\mathrm{L} \rightarrow \mathrm{Ag}_{2} \mathrm{Nd}\right)$ in the $\mathrm{Nd}-\mathrm{Ag}$ system [103].

The XRD data presented in Figure 55 indicates that this texture decreases as wheel speed is increased. It is particularly visible in the (204) peak, which is of the greatest intensity in the $5 \mathrm{~m} / \mathrm{s}$ trace, but relatively low intensity in the $20 \mathrm{~m} / \mathrm{s}$ ribbon. This implies a dependence of the final texture on the initial solidification microstructure. The exact nature of the initial solidification structure, however, remains to be determined.

\section{Solidification Pathway}

The formation of an unusual texture and the fine dispersion of precipitates are indicative of a change in solidification pathway of the alloy with small additions of Ag. At this point the exact pathway from melt to solidified ribbon is unclear due to the complexity of the system, but several features of the phases and morphologies observed here can provide some clues. shows that a fine, equiaxed structure is initially formed next to the quench surface. TEM images indicate that $\mathrm{Ag}$ is rejected from the matrix and can be found in at grain boundaries and triple junctions, as well as in $\mathrm{AgNd}$ precipitates within the matrix, itself (see Figure 62). The presence of these precipitates in the matrix indicate either solute trapping of the silver within the grains due to the high thermal gradient and the interfacial velocity it implies [74], or that a degree of solubility of the $\mathrm{Ag}$ in a high temperature phase may be present. Upon cooling, it would be rejected from the 2-14-1 matrix, forming the fine precipitates.

As the solidification front velocity slows (presumably), the sudden start of columnar dendritic/cellular growth occurred apparently. This can be seen in and 
Figure 58. The interface between the fine equiaxed region and the columnar region very consistently is located approximately $20 \mu \mathrm{m}$ from the surface in contact with the wheel in the $5 \mathrm{~m} / \mathrm{s}$ ribbons. The columnar structure was seen to extend through the remainder of the structure with only a moderate degree of coarsening. In Figure 53, the location of the centers of the columns can be identified by the centers of the 3pointed "star-shaped" dark contrast regions. Observation of these regions in the TEM (Figure 63) suggests they are simply denuded zones in which the fine precipitates are not present and their composition (by EDS in the STEM) is identical to the 2-14-1 phase. The morphology of these "stars" is indicative of dendrite cores, but this type of growth morphology is not consistent with any crystallographic direction of the equilibrium 2-14-1 crystal structure. The apparent secondary arms of the dendrites (the branches of the "stars") form $120^{\circ}$ angles with one another, suggesting that the easy growth directions of the dendrites form a $120^{\circ}$ pattern (a possible indication of trigonal symmetry). Lines of the fine precipitates are seen to extend from the secondary arms, indicating rejection of $\mathrm{Ag}$ and $\mathrm{Nd}$ between tertiary arms.

It is also likely that $\mathrm{Ag}, \mathrm{Nd}$, and $\mathrm{B}$ are rejected from the primary dendrites, since phases containing these elements can be found along much of the interdendritic regions. The faceted morphology and gross segregation observed in these phases indicate possible liquid immiscibility in the interdendritic regions as additional $\mathrm{Ag}$ and $\mathrm{B}$ are rejected from the matrix. Further evidence of this is that a large liquid miscibility gap is present in both the $\mathrm{Ag}-\mathrm{Fe}$ and $\mathrm{Ag}-\mathrm{B}$ systems.

It is currently unclear which phase is the primary phase that initially forms the dendrite cores since equilibrium 2-14-1 phase is not likely. There are a number of other possible transient phases indicated by the various equilibrium phase diagrams of components in the system, including $\mathrm{RE}_{2} \mathrm{Fe}_{17}$ and $\mathrm{REFe}_{3}$. However, direct evidence of these phases has not been observed in the post-solidification ribbons. Of course, any transient phases that are metastable would not be possible to detect unless an in situ crystal structure probe was used during the solidification reaction. 


\section{Conclusions}

The addition of $\mathrm{Ag}$ was seen to greatly affect the solidification microstructure of $\left(\mathrm{Y}_{0.55} \mathrm{Nd}_{0.45}\right)_{2.2} \mathrm{Fe}_{14} \mathrm{~B}_{1.1}$ alloys melt-spun at low to moderate wheel speeds. A columnar dendritic structure of $\mathrm{RE}_{2} \mathrm{Fe}_{14} \mathrm{~B}$, characterized by 3 -fold symmetry in the secondary arms was stabilized through the majority of the ribbon thickness. Between the columns, $\mathrm{Ag}_{2} \mathrm{Nd}$ precipitates were found in a "bead-like" morphology with a second phase dispersed between the precipitates (likely $\left.\mathrm{RE}_{1.1} \mathrm{Fe}_{4} \mathrm{~B}_{4}\right)$. Within the grains, fine precipitates $(\sim 2-50 \mathrm{~nm})$ of $\mathrm{AgNd}$ were found dispersed through the matrix. In the interdendritic regions, several additional phases were observed, including $\mathrm{Nd}$-rich, $\mathrm{AgNd}$, and likely $\mathrm{RE}_{1.1} \mathrm{Fe}_{4} \mathrm{~B}_{4}$. The segregation seen in these regions indicates possible liquid immiscibility.

X-ray diffraction measurements indicated an alteration of texture within the ribbons, characterized by enhanced (204), (214), and (105) peaks. As wheel speed was increased, the texture was seen to decrease as the structure transitioned to an isotropic structure, an indication that the texture is largely based on the primary solidification structure generated at lower solidification velocities.

\section{Acknowledgements}

This work was supported by the US Department of Energy-Office of Energy Efficiency and Renewable Energy, Vehicle Technology Office, USDRIVE, and the PEEM program, under Contract No. DE-AC02-07CH11358 for the operation of Ames Laboratory (USDOE).

FIB work was completed by Jatuporn Burns at the Center for Advanced Energy Studies (CAES) in Idaho Falls, ID. LEAP data was collected by Dr. Yaqiao Wu at CAES. TEM work was done by Dr. Qingfeng Xing and Emma White. Dan Cavanaugh and Nick Fries were instrumental in sample preparation. 


\section{Chapter 8 - General Conclusions and Future Directions}

In this work, several different methods of microstructure control for $\mathrm{RE}_{2} \mathrm{Fe}_{14} \mathrm{~B}$ alloys were investigated. These include controlled devitrification through applied pressure (as presented in chapter 4), modification of alloy design (by incorporating a segregating element, $\mathrm{Ag}$ ) to stabilize columnar structures during melt-spinning and selection of suitable accompanying solidification parameters (as described in chapters 6 and 7), and modification of the wheel temperature and surface finish during melt-spinning (as described in chapter 5). Despite the fact that, in their current form, none of the methods studied produced a material suitable for use in high temperature anisotropic bonded magnets; all of the methods were seen to significantly alter the final microstructures. The alterations proved promising methods of control for creating suitable particulate. It is my recommendation that, of the methods studied here, the avenue that should be pursued is low wheel speed melt-spinning of alloys containing segregating additives. A secondary method for controlling microstructure is the application of uniaxial pressure upon devitrification, though significant work would be needed to scale the process to a large enough size for cost-effective general production.

After suitable particulate is formed, the particulate would then be crushed to a moderate size (on the order of $30-100 \mu \mathrm{m}$ particle size) and blended with a high temperature polymer. Suitable polymers include high temperature epoxies and polyphenylene sulfide (PPS). The particulate would then be aligned in a magnetic field while the polymer is cured.

\section{Application of uniaxial pressure during devitrification}

Application of uniaxial pressure (via vacuum hot pressing) during devitrification of mostly-amorphous $\mathrm{RE}_{2} \mathrm{Fe}_{14} \mathrm{~B}+\mathrm{TiC}$ precursor material provided a nanocrystalline textured microstructure. The density of defects, however, was very high and likely caused a loss of coercivity in the material. The formation of the defect structure is unprecedented, as the structure has no active slip systems until temperatures upwards of $900^{\circ} \mathrm{C}$ are reached. We have surmised that the defects 
form through the physical restriction of growth due to the applied pressure, while diffusion is limited by the relatively low temperature processing. Despite this high defect density, however, it is likely that annealing heat treatments would allow the particulate to recover and regain the lost coercivity, while hopefully maintaining the induced texture.

This method provided one of the most promising microstructures of the methods investigated. Despite this, it is not recommended for pursuit due to the difficulty in obtaining significant quantities of the material (most trials resulted in batch sizes on the order of 10's of milligrams). It is unlikely that, in its current form, this process would be scalable to the size necessary for commercialization, and is therefore not recommended for pursuit unless other methods prove insufficient. Additionally, if the process could be scaled appropriately, there is no guarantee that it would prove to be any more economically viable than the die-upset process.

\section{Modification of wheel temperature and surface finish during melt-spinning}

Modification of the wheel temperature and surface finish were found to have a large impact on the ability to form amorphous content in the Nd-Fe-B-Ti-C alloy studied. While modification of these parameters did not directly result in the formation of anisotropic material in the conditions observed, the findings can be used as another tool to control the melt-spinning process.

The use of a heated wheel surface during melt-spinning was found to have significant impact on the phases achieved in the resulting ribbons. Meltspinning on a $200^{\circ} \mathrm{C}$ wheel increased the degree of glass formation in the ribbon compared to room temperature or $100^{\circ} \mathrm{C}$ wheels. At first glance, this finding appears counterintuitive as a heated wheel should decrease the thermal gradient and reduce the thermal conductivity of the wheel. After observation of the ribbon surface that was in contact with the wheel, it was concluded that the heated wheel surface affected the thermal interface between the melt and the wheel, specifically the ability of the melt to wet the wheel surface. 
The exact mechanism of this effect has yet to be determined, but several possibilities have been discussed. By allowing the melt to stay liquid (in a lower viscosity or surface tension state) for an extended period of time, the melt could better conform to the wheel surface, effectively increasing the surface area and thermal conductance across the interface. It was also noted that the oxidation state of the wheel might have some impact on the wetting behavior. In the temperature regimes that the wheel was held, the oxidation behavior of $\mathrm{Cu}$ (at least in ambient atmosphere) is somewhat complex, with several stable or metastable oxide stoichiometries.

Another finding in chapter 5 was that surface finish played a significant role in the ability to form amorphous material as well. The amorphous content was seen to decrease when the wheel surface was finished using sandpaper with decreasing abrasive particle size. A similar effect on the wetting behavior was seen here. An attempt to model the wetting behavior and its effect on heat transfer during melt-spinning was attempted and a summary of this can be found in appendix B.

Increasing the heat extraction rate, however, does not appear to aid in the formation of a textured microstructure. Rather, the effect of a heated wheel surface decreases the observed texture at low wheel speeds [120]. While the decreased texture is unfavorable for solidifying directly to the desired microstructure, the increased glass formation achievable through this process could aid in the production of suitable precursor material for use in controlled devitrification experiments. This process also offers additional degrees of control that could be used in the formation of traditional "overquenched" microstructures as desired for nanocrystalline isotropic magnet particulate. By achieving the greater cooling rates, it may be possible to remove (either partially or wholly) many of the grain refiners used in these isotropic alloys. It may be possible (through proper selection of wheel surface characteristics, material, and temperature) to provide conditions where the fine equiaxed zone thickness could be minimized relative to the amount of columnar structure generated. This would be greatly useful for generating anisotropic particulate. 
It is, however, important to note that the increased degree of wetting seen at fine surface finishes and high wheel temperatures can result in adherence of the melt to the wheel surface. This can be a problem for maintaining consistency throughout a run. As a result, the parameters must be carefully chosen and active cleaning of the wheel may be necessary.

Several different experiments for future study are apparent. Further understanding of the exact mechanism by which the interfacial heat transfer coefficient is modified by the wheel temperature would further help in selecting the appropriate temperature. One possible mechanism surmised was the presence of varying stoichiometry of $\mathrm{Cu}$ oxide present on the wheel. One could study this by a) characterizing the oxide present on the wheel at varying temperatures, b) melt-spin on a pre-oxidized wheel, or c) changing wheel material (e.g. electroplating a different material on the surface).

Low wheel speed melt-spinning and the use of segregating elements in the alloy Moving forward, the recommended pathway to pursue is to form the texture upon solidification of the alloy during melt-spinning. This requires moderately high cooling rates to obtain the appropriate interfacial velocity; it must be slow enough to maintain an oriented columnar structure, but fast enough to maintain small grain size. The ability to do so likely requires the use of a segregating addition to the base alloy in order to increase interfacial instability at increasing solidification front velocities.

The work presented here focused on the use of $\mathrm{Ag}$ as an additive to the alloy, as it was seen to have the greatest effect on stabilization of a columnar microstructure of the elements tested and resulted in the alteration of the texture of the ribbon. Further discussion of initial studies of the other elements tested will be presented in appendix $\mathrm{A}$.

Ag provided the most promising microstructure of the elemental additions observed. The columnar structure was stabilized through almost the entire ribbon thickness. Further characterization of the structure was continued and several 
surprising results were found. The texture was seen to be altered from the c-axis texture observed in all the other solidified ribbons. This indicates a change in the texturing mechanism from what was originally proposed by Kramer et al. for low wheel speed ribbons. Since the texture is linked to the solidification pathway, it is clear that the presence of $\mathrm{Ag}$ alters the solidification microstructure significantly. In all the other microstructures observed (with the possible exception of $\mathrm{Ta}$ ), the segregating elements were seen to be pushed to the grain boundaries. In the case of $\mathrm{Ag}$, however, a significant portion of the $\mathrm{Ag}$ was found within the matrix in the form of precipitates.

One feature of the $\mathrm{Ag}$ addition not present with the other additions observed is the presence of large miscibility gaps in the $\mathrm{Ag}-\mathrm{Fe}$ and $\mathrm{Ag}-\mathrm{B}$ systems. The increased segregation, even well into the liquid, can provide some unique features. The final solidified microstructure shows segregation on the nanometric scale with the distribution of the fine AgNd precipitates. With the presence of a strong driving force to segregate, it is likely that the precipitates will display high thermal stability as there is little driving force for the Ag to diffuse back into the $\mathrm{Fe}$ rich matrix, even upon melting. This has some possible implications for the formation of magnetic domain pinning sites. Work on $\mathrm{Nb}$-containing alloys indicated an increase in coercivity with the formation of similar fine precipitates. It was shown through Lorentz force microscopy that the magnetic domains interacted with the precipitates, a likely indication of domain wall pinning. Further understanding of the mechanism of formation of these precipitates might allow for increased coercivity magnets. By increasing coercivity, Dy additions needed for high temperature applications may be able to be reduced.

The alteration of texture also has implications. The observed texture in the Ag-containing ribbons is certainly not ideal. However, a strong ability to form a textured structure with a fraction of an atomic percent Ag suggests a powerful ability to control the growth of the alloy. Further understanding of the mechanism could provide additional processing routes for forming aligned structures. Studies further characterizing the texture are underway, as well as studies investigating the initial 
phase of the dendrite cores. One possible way to do this would be through in situ high energy $x$-ray diffraction, where the material would be characterized during solidification from the melt. Hopefully the metastable phase would be present at the relatively low cooling rates.

In all of the structures utilizing additives, the scale seen is currently too large to adequately maintain coercivity and further refinement is necessary. A possible direction that could be taken is the use of multiple additives to further increase interfacial instability, though that would add an additional degree of complexity to an already complex system. Further optimization of the parameters used in melt-spinning (i.e. surface finish or wheel temperature) could also help achieve the desired microstructures. 


\section{Acknowledgements}

A number of people were very important in the completion of this work, whether through aiding in data collection, discussion of the work, or general moral support. I could not have completed it without their help.

Dan Cavanaugh, Kevin Dennis, Dr. Wei Tang, Dr. Yaqiao Wu, Dr. Qingfeng Xing, Fran Laabs, Jim Anderegg, Alfred Kracher, Warren Straszheim, Nick Fries, Sam Hanselman, Allison Loecke, and Eric Harms all provided a great deal of help in sample preparation and characterization over the course of this work.

Several professors have been particularly influential in my time at lowa State: Dr. Scott Chumbley, Dr. Ralph Napolitano, Dr. Alan Russell, Dr. Richard LeSar, Dr. Alan Constant, Dr. Larry Genalo, Dr. Loren Zachary, and Dr. Mani Mina.

The "Powder Group" has been a great help in offering the chance to practice presenting work and helping refine ideas. Members have included Dr. Joel Rieken, Dr. Eren Kalay, Dr. Andy Heidloff, Dave Byrd, Adam Boesenberg, Ben Rattle, Jason Walleser, Scott Long, Emma White, Andrew Steinmetz, Alex Spicher, John Meyer, and Greg Vetterick.

I would also like to thank my program of study committee for their guidance and time refining this work: Dr. Matthew Kramer, Dr. R.W. McCallum, Dr. Ralph Napolitano, Dr. Frank Peters, and especially my advisor, Dr. Iver Anderson.

I am particularly thankful for the support of my family throughout my entire schooling, and in all areas of my life. They have challenged me, they have encouraged me, and they have formed who I am today. Thank you George Oster, Vicky Thorland-Oster, Jessica Oster.

Most importantly, I would like to acknowledge my wife, Sasha. Her encouragement was a large part of the reason I entered the Ph.D. program. Her zest for learning (and teaching) is an inspiration to me. Going through graduate school, taking classes together, and learning together has been one of the most enjoyable experiences l've had. 
This work was performed at the Ames Laboratory under contract number DE-AC02-07CH11358 with the U.S. Department of Energy. Funding was provided through the Energy Efficiency and Renewable Energy office US-DRIVE program.

$[121,122]$ 


\section{Appendix A}

This appendix contains additional experimental results that were not presented earlier in the document. These are results that either were initial results used to screen samples or initial results that were very recently taken and have not yet been published.

\section{A.1 - Initial studies on other additives}

Several other elements showing little solubility in the base elements of the alloy were identified as possible additives. Initial work has been done to screen them through SEM cross-section micrographs. Small additions (0.5-1 at. \%) of the chosen elements were made. Cu was the first element tested. It was found to stabilize the columnar structure in some regions, but not others (see Figure 66). $\mathrm{Zr}$, Hf, and $\mathrm{Ta}$ were also tried. $\mathrm{Zr}$ was not found to stabilize the columnar structure (see Figure 67); nor did Hf. Rather, significant dendrite secondary arm formation was observed. Ta was found to stabilize the columnar structure in some regions, similar to the effects of $\mathrm{Cu}$. Further investigation of Ta may be worthwhile.
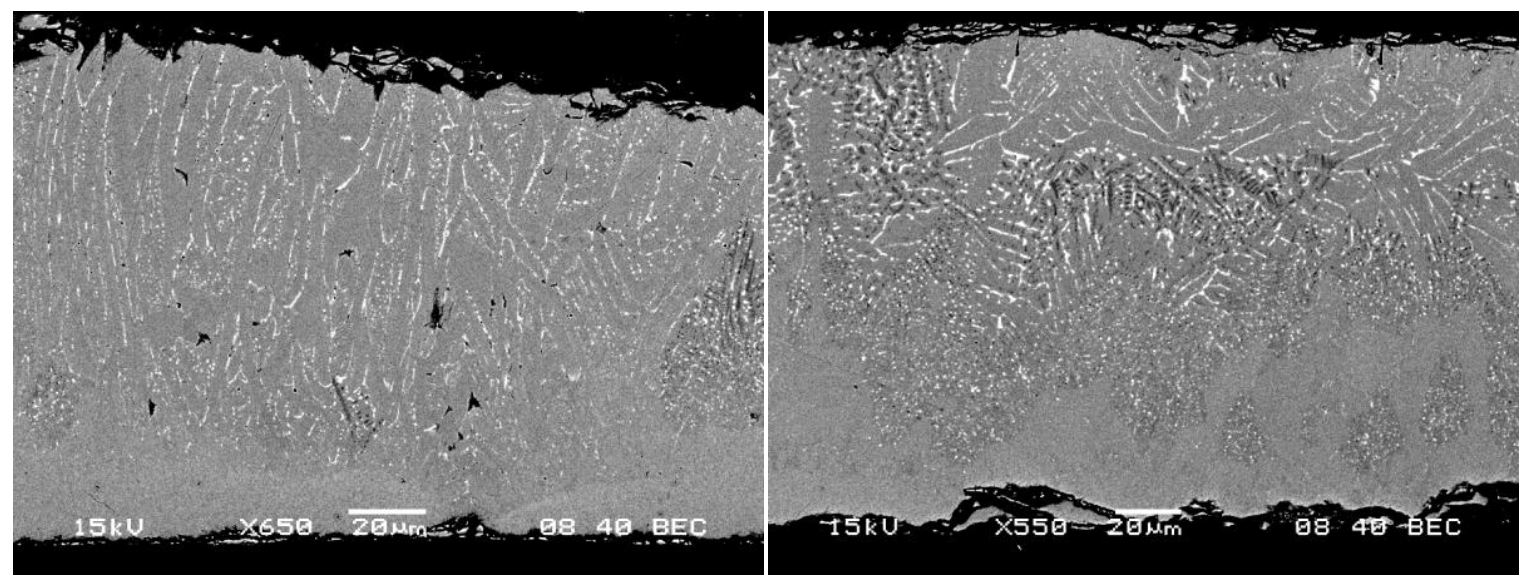

Figure 66 - BSE micrographs of $\left(\mathrm{Nd}_{0.4} \mathrm{Y}_{0.4} \mathrm{Dy}_{0.2}\right)_{2.15} \mathrm{Fe}_{14} \mathrm{~B}_{1.1} \mathrm{Cu}_{0.15}$ melt-spun at $5 \mathrm{~m} / \mathrm{s}$ showing regions where the columnar structure was stabilized (left) and where the columnar structure broke down (right). 


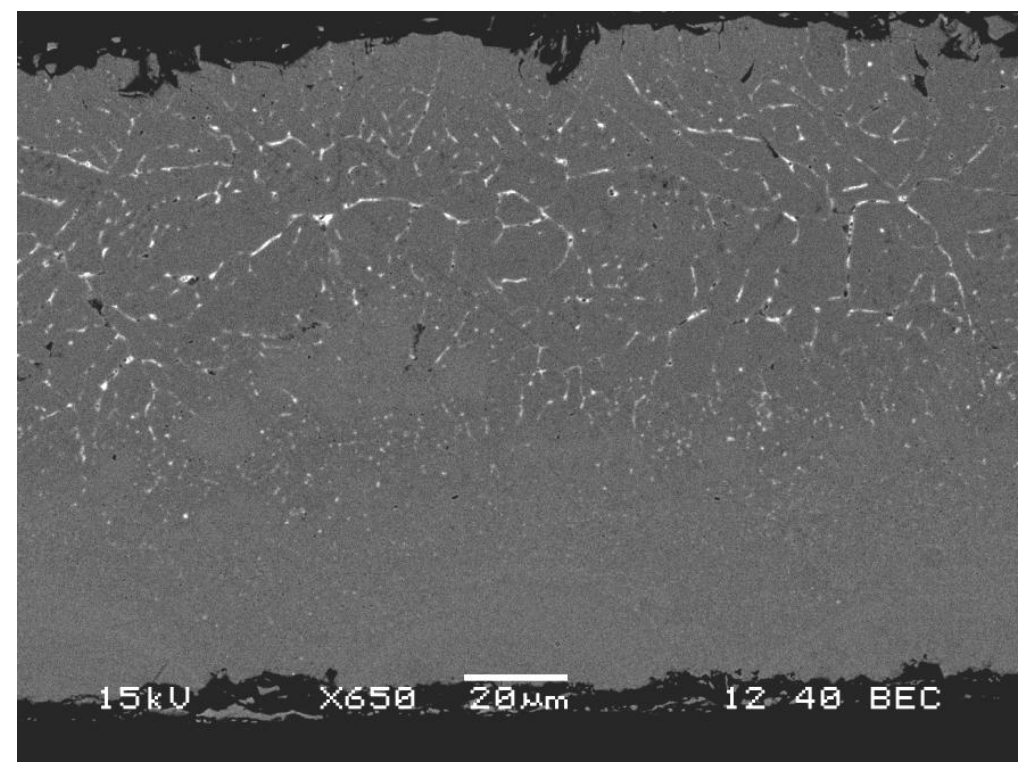

Figure 67 - BSE micrograph of $\left(\mathrm{Nd}_{0.4} \mathrm{Y}_{0.4} \mathrm{Dy}_{0.2}\right)_{2.15} \mathrm{Fe}_{14} \mathrm{~B}_{1.1} \mathrm{Zr}_{0.1}$ melt-spun at $5 \mathrm{~m} / \mathrm{s}$

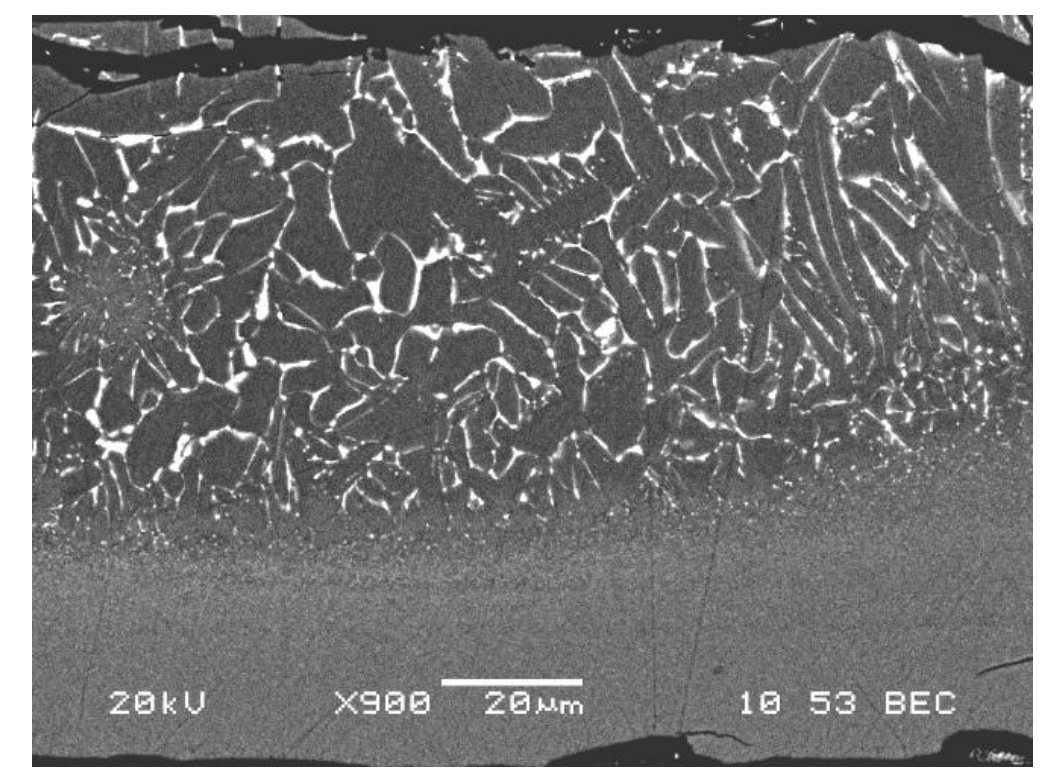

Figure 68 - BSE micrograph of $\left(\mathrm{Nd}_{0.45} \mathrm{Y}_{0.55}\right)_{2.2} \mathrm{Fe}_{14} \mathrm{~B}_{1.1} \mathrm{Hf}_{0.1}$ melt-spun at $5 \mathrm{~m} / \mathrm{s}$ 


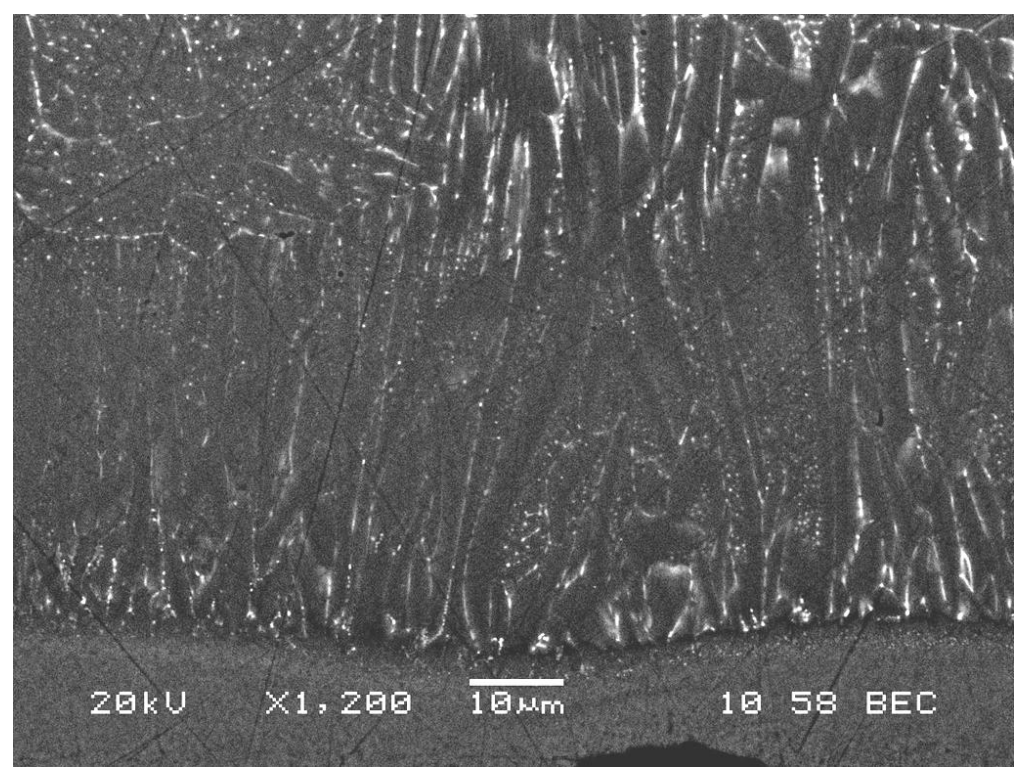

Figure 69 - BSE micrograph of $\left(\mathrm{Nd}_{0.45} \mathrm{Y}_{0.55}\right)_{2.2} \mathrm{Fe}_{14} \mathrm{~B}_{1.1} \mathrm{Ta}_{0.1}$ melt-spun at $5 \mathrm{~m} / \mathrm{s}$

To further investigate the effects of having an element that segregates as strongly as $\mathrm{Ag}$, $\mathrm{Bi}$ was identified as another possible additive. Initial work on a $\mathrm{Bi}$ added alloy has been started. $\mathrm{Bi}$ is similar to $\mathrm{Ag}$ as an additive in that a miscibility gap occurs in the Bi-Fe system. In addition, the Bi-RE system shows virtually zero solubility. $\mathrm{Bi}$ is, however, a much lower melting element and could allow for liquid to be present to very low temperatures. 


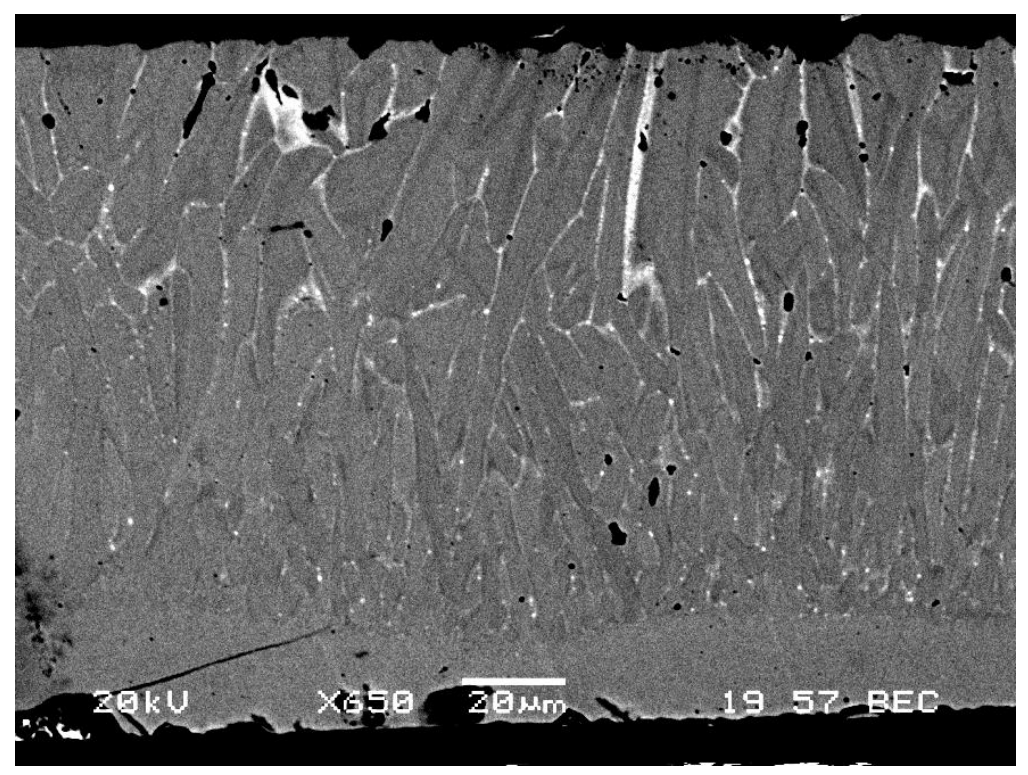

Figure 70 - BSE micrograph of $\left(\mathrm{Y}_{0.55} \mathrm{Nd}_{0.45}\right)_{2.2} \mathrm{Fe}_{14} \mathrm{~B}_{1.1} \mathrm{Bi}_{0.15}$ melt-spun at $5 \mathrm{~m} / \mathrm{s}$

\section{A.2 - Orientation imaging microscopy study of Nd-Y-Fe-B-Ag alloys}

Orientation imaging microscopy (OIM) was used to characterize the texture seen in the XRD patterns observed in chapters 6 and 7 . The data collected is very noisy, but the grain structure can be seen. It is likely that this noise is caused, in part, but the presence of the fine $\mathrm{Ag}-\mathrm{Nd}$ precipitates in the matrix.

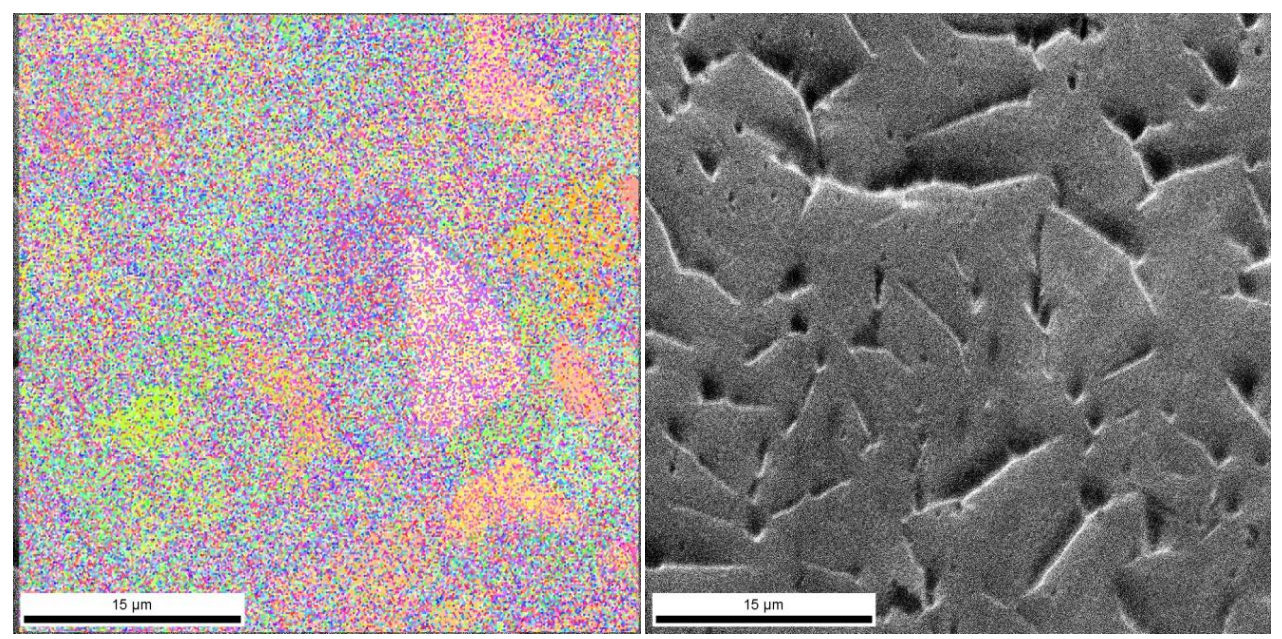

Figure 71 - OIM image (left) taken from the area shown in the micrograph on the right. The grain structure can be seen, but significant amounts of noise are present in the data. 
The pole figure indicates that the basal plane of the crystal structure is likely canted from the plane of the ribbon at an angle between about $40^{\circ}$ and $60^{\circ}$, but the angle is randomly oriented in the plane of the ribbon. The inverse pole figure indicates that the columns are mostly oriented at angles near the $<101>$ and $<102>$ directions.

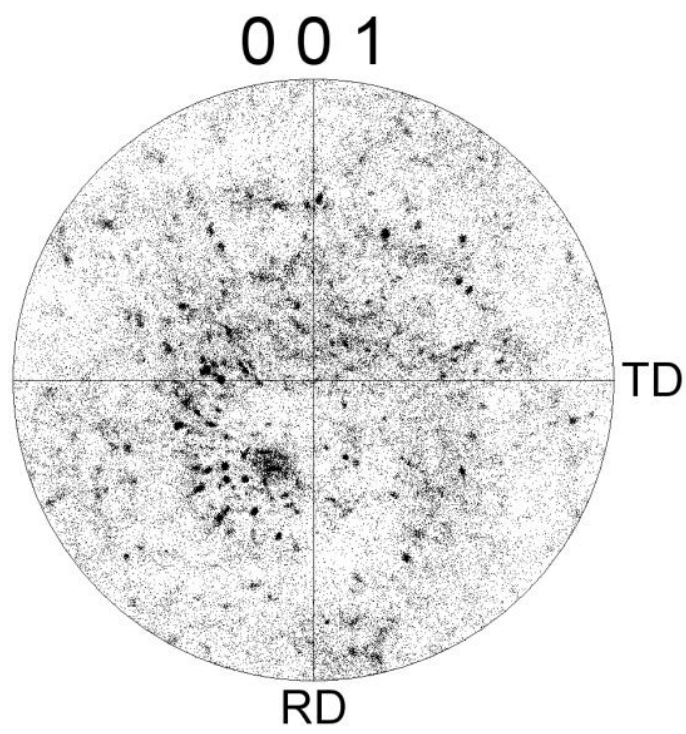

[001]

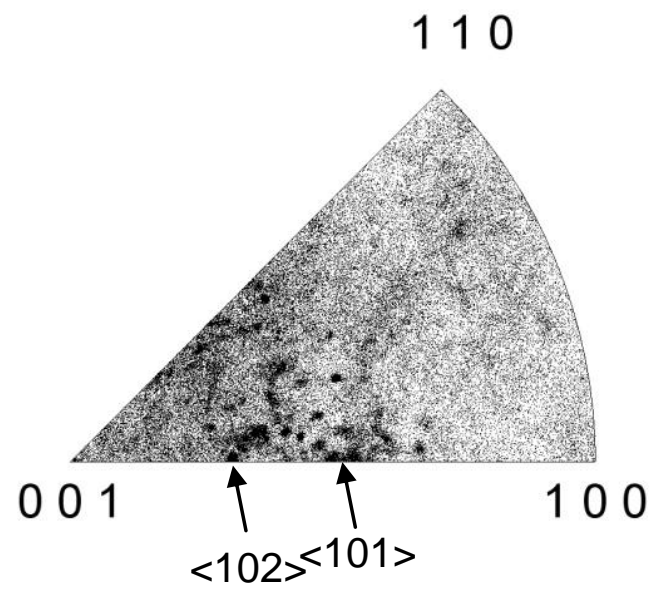

Figure 72 - Pole figure (left) and inverse pole figure (right) of the data from Figure 71.

Further work is needed to fully characterize the texture. 


\section{Appendix B - A simple model for wheel profile and its effects on heat flow during melt-spinning}

This appendix discusses the attempt to create a simple model to evaluate heat flow and interfacial heat transfer coefficient during melt-spinning.

\section{Creation of a model wheel profile}

Contact area between the melt and the wheel makes a significant impact on the total heat flow in the system. Since grooves form in the wheel surface from the abrasive used to finish the wheel, a two dimensional projection of the surface can be modeled, to a first approximation, as a cosine function:

$$
y=A \cos \left(\frac{2 \pi}{\lambda} x\right)
$$

Where $A$ is the amplitude of the function (half of the groove depth) and $\lambda$ is the wavelength (spacing between grooves). A general expression for the length of the curve can be derived using the Pythagorean theorem. For a given segment:

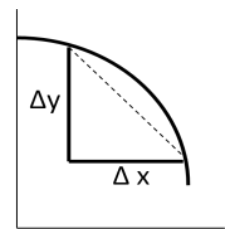

$$
\Delta L=\sqrt{\Delta x^{2}+\Delta y^{2}}
$$

As $\Delta \mathrm{x}$ and $\Delta \mathrm{y}$ approach zero, the expression can be written in differential form and summed over the area in question:

$$
L=\int_{0}^{B} \sqrt{\left(\frac{d y}{d x}\right)^{2}+1} d x
$$

Substituting the derivative of the cosine function into the expression above: 


$$
L=\int_{0}^{B} \sqrt{\left[\frac{A * 2 \pi}{\lambda} * \sin \left(\frac{2 \pi}{\lambda} x\right)\right]^{2}+1} d x
$$

Where $A=$ amplitude of the groove ( $1 / 2$ groove depth), $\lambda=$ distance between grooves (i.e. wavelength), and B is the boundary condition, calculated as follows:

$$
B=\frac{\lambda}{2 \pi} \cos ^{-1}\left(\frac{A-d}{A}\right)
$$

Where $d=$ penetration depth of liquid (if the liquid fully penetrates into the groove, $B=N / 2$ ). The solution to the integral is an elliptic integral of the $2^{\text {nd }}$ form, which can be solved computationally using Matlab. A schematic is shown below in Figure 73. The red line represents the contact area calculated inside the integral (it is then doubled to account for both sides of the sinusoid).

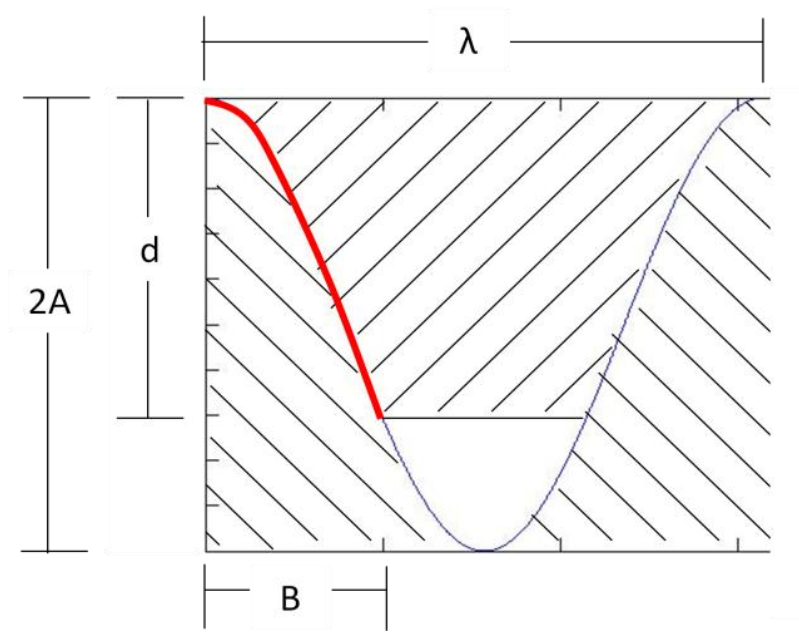

Figure 73 - schematic of scenario in which surface contact area was calculated

The relationship between groove wavelength and groove depth has been shown to be $\lambda \approx 9^{*}$ depth $[123,124]$. The width of groove has also been shown to be approximately $1 / 10^{\text {th }}$ of the abrasive particle diameter [125]. If the relationship between groove depth and groove width is held constant and the melt is assumed to fully penetrate the groove, the surface area does not change with increasing depth. However, if the liquid is assumed to penetrate to a certain level (e.g. $50 \mathrm{~nm}$ from the 
bottom of the groove), the contact area varies with wavelength. This is shown below in Figure 74.

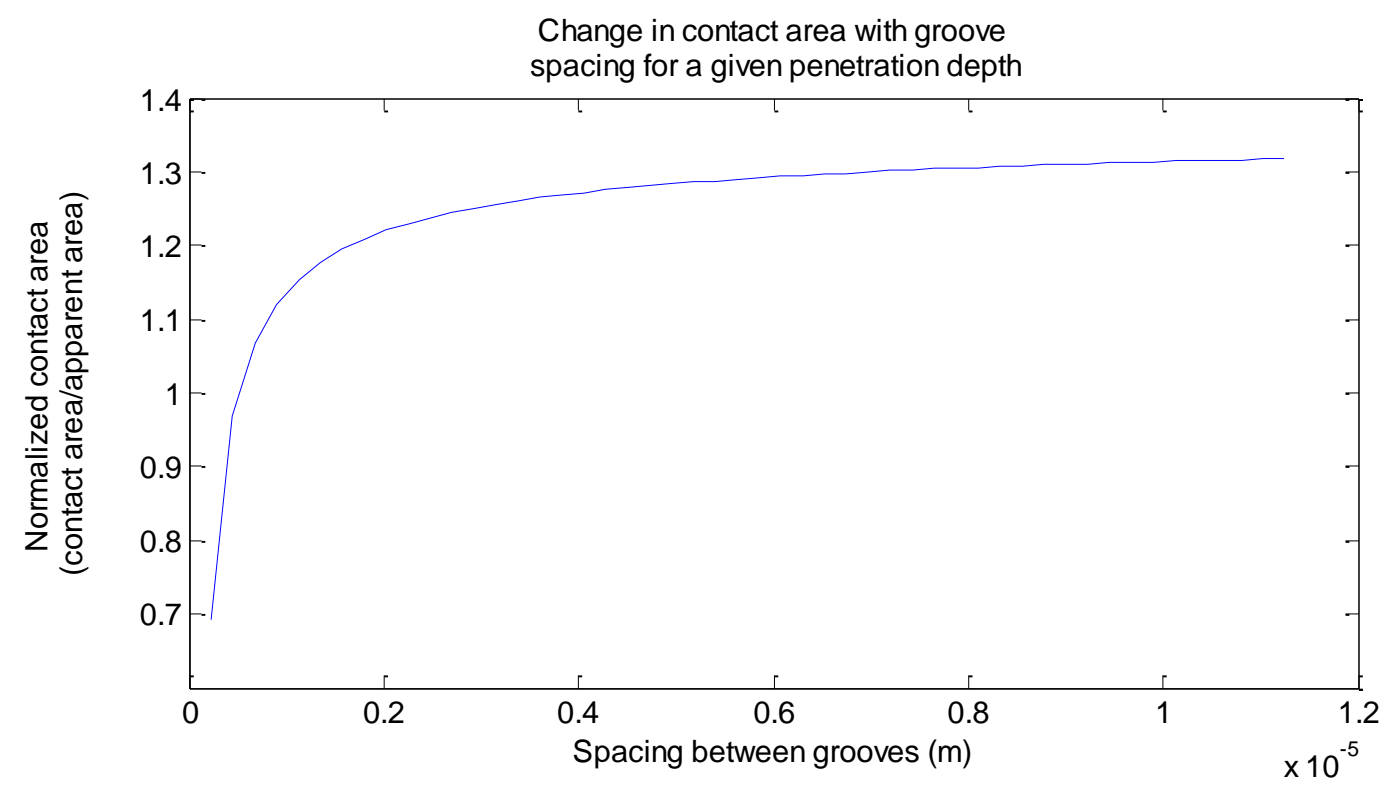

Figure 74 - Change in contact area observed with increasing groove spacing for liquid penetrating to $50 \mathrm{~nm}$ from the bottom of the groove.

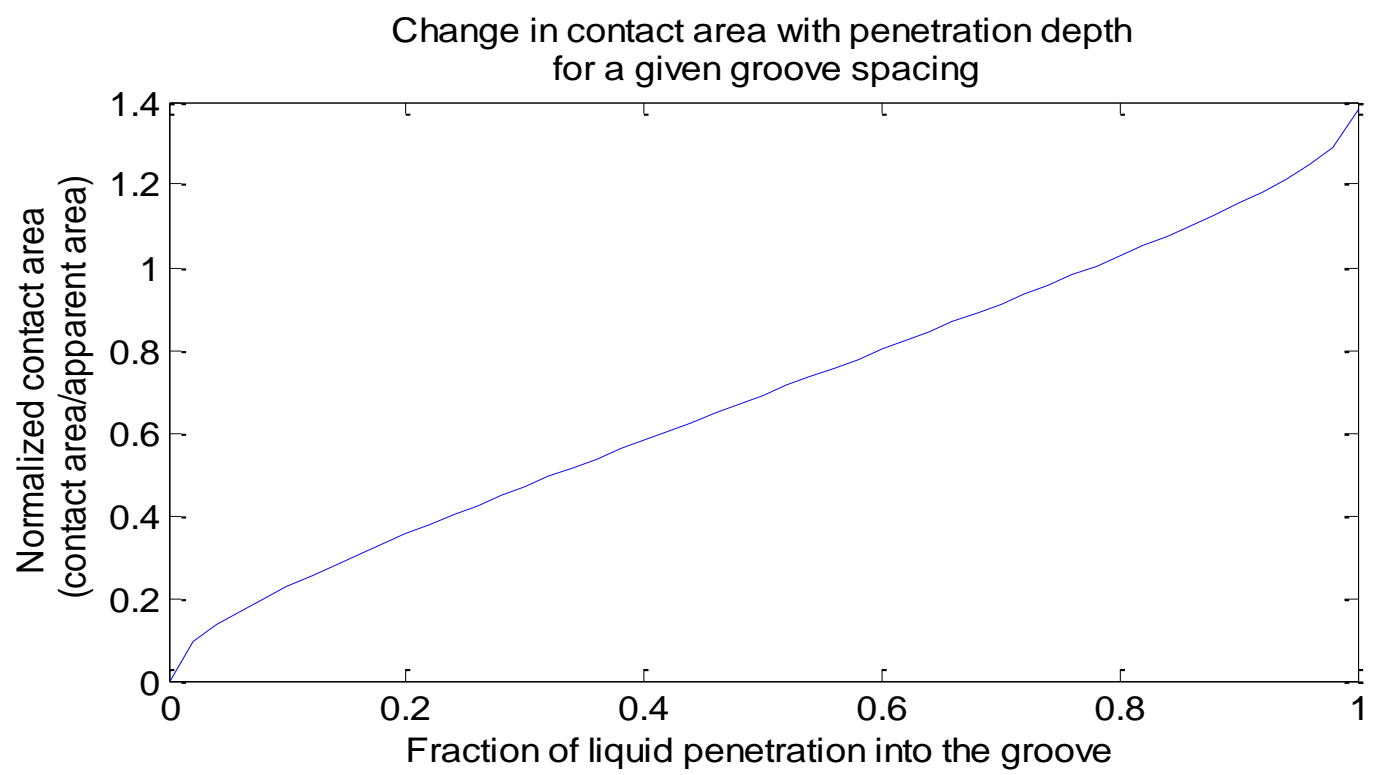

Figure 75 - Change in normalized contact area with penetration depth for a given groove spacing (and amplitude). 


\section{Creation of a 2-D finite differencing simulation}

Using this idea that the difference in relative liquid penetration depth could account for the change in heat transfer observed when finer grits were used to finish the wheel surface (see chapter 5), a simple finite differencing simulation modeling 2dimensional heat flow between the liquid and the wheel was created. The model assumed that the melt initially started at a uniform temperature of $1450 \mathrm{~K}$. The wheel temperature was set to either $300 \mathrm{~K}$ or $475 \mathrm{~K}$ (however, room temperature values for thermal conductivity and heat capacity were used in order to simplify computation). Repeating boundary conditions were used on the left and right hand sides of the profile. The top and bottom rows of elements were maintained at the initial temperature conditions throughout the simulation. A schematic of the initial conditions is shown below in Figure 76 (the schematic has been repeated several times in order to show the repeating boundary conditions.
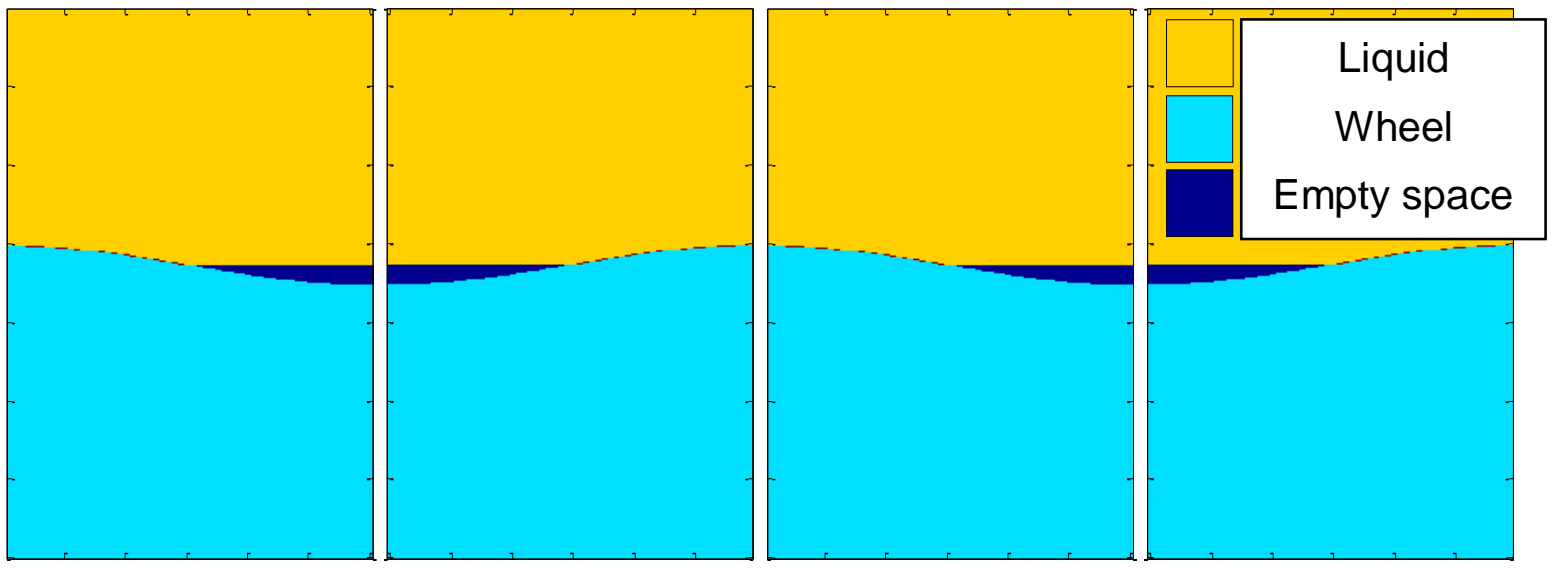

Figure 76 - Example profile used for the heat flow simulation

Fourier's law of conduction was used to measure heat flow between the elements:

$$
q=-k \nabla T
$$

Where $q$ is the heat flow (in $\mathrm{W} / \mathrm{m}^{2}$ ), $\mathrm{k}$ is the average thermal conductivity of the elements in question, and $\nabla T$ is the thermal gradient. An example of the specific 
formulation used is shown below. This example calculates the net heat change in element B4:

$q=-\left[\frac{k_{B 3}+k_{B 4}}{2}\left(\frac{T_{B 4}-T_{B 3}}{a}\right)+\frac{k_{B 5}+k_{B 4}}{2}\left(\frac{T_{B 4}-T_{B 5}}{a}\right)+\frac{k_{A 4}+k_{B 4}}{2}\left(\frac{T_{B 4}-T_{A 4}}{a}\right)+\frac{k_{C 4}+k_{B 4}}{2}\left(\frac{T_{B 4}-T_{C 4}}{a}\right)\right]$

Where the subscripts represent the elements of the grid shown below and a is the cell size:

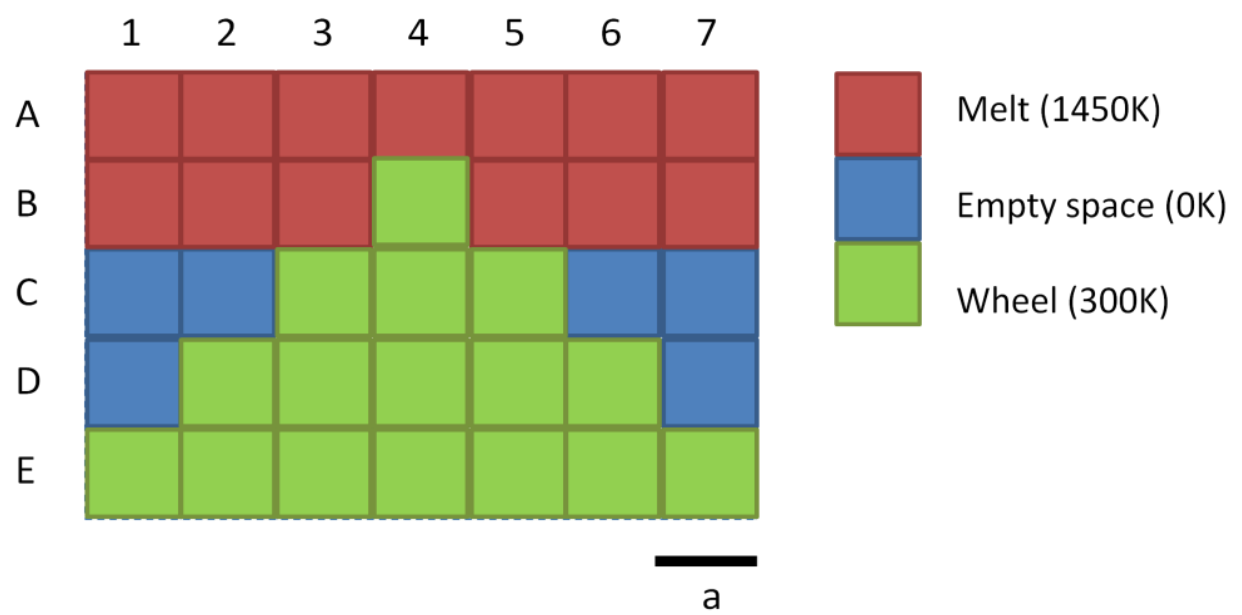

Figure 77 - Schematic used for the sample calculation above.

This is then repeated for all remaining elements of the matrix. The change in heat is then added to the enthalpy value for each element and temperature is recalculated via the expression:

$$
T=\frac{H}{C * V}
$$

Where $\mathrm{T}$ is temperature (in $\mathrm{K}$ ), $\mathrm{H}$ is enthalpy (in J), $\mathrm{C}$ is volumetric heat capacity (in $\mathrm{J} / \mathrm{m}^{3}-\mathrm{K}$ ), and $\mathrm{V}$ is volume (in $\mathrm{m}^{3}$ ). It should be noted that this expression assumes that no heat is released upon crystallization (i.e. the melt is treated as fully amorphous regardless of temperature/cooling rate). 
A flow chart of the program is shown below in Figure 78.

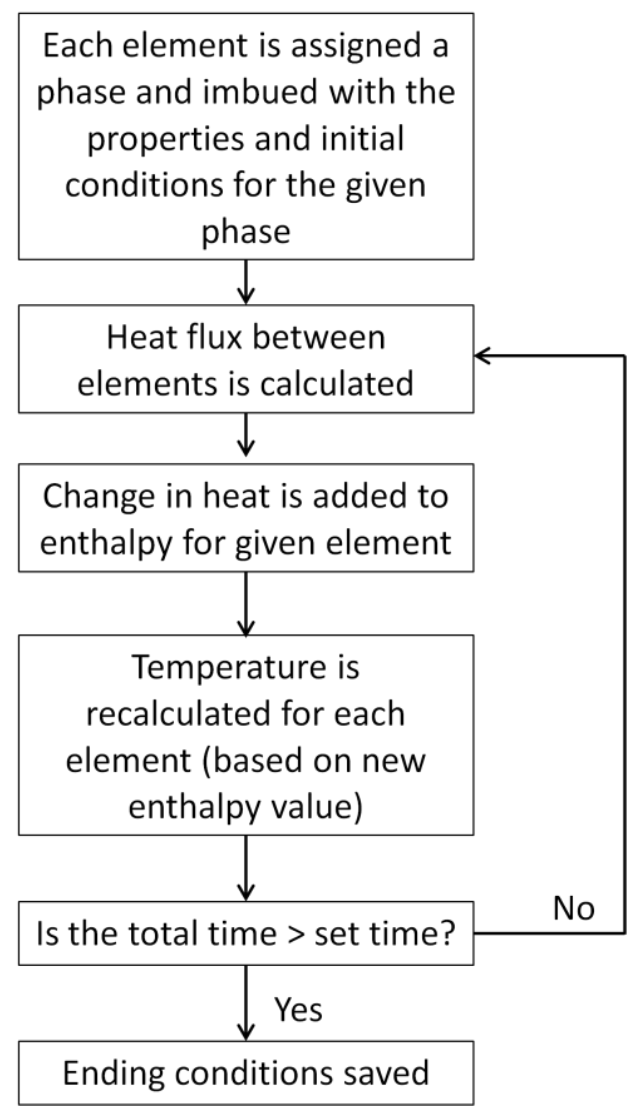

Figure 78 - Flow chart of simulation.

The simulation was used to determine the effects on heat flow across the interface as a function of fill depth for both a room temperature wheel and a $200^{\circ} \mathrm{C}$ wheel. One set of results is shown below in Figure 79. Table II shows the parameters used to obtain these results. 
Table 2 - Simulation parameters for the results shown in Figures 82-84

\begin{tabular}{|c|c|c|}
\hline Parameter & Wheel & Melt \\
\hline Wavelength (\# of elements) & 250 & 250 \\
\hline Height of Phase (\# of elements) & 400 & 300 \\
\hline $\begin{array}{c}\text { Size of element edge }(\mathrm{m}) / \\
\text { Volume of element }\left(\mathrm{m}^{3}\right)\end{array}$ & \multicolumn{2}{|c|}{$4 \times 10^{-8} / 6.4 \times 10^{-23}$} \\
\hline Initial Temperature (K) & 300 or 475 & 1450 \\
\hline Thermal Conductivity $(\mathrm{W} / \mathrm{m}-\mathrm{k})$ & 350 & $3.71 \times 10^{6}$ \\
\hline Vol. Specific Heat $\left(\mathrm{J} / \mathrm{m}^{3}-\mathrm{K}\right)$ & $3.86 \times 10^{6}$ & $10^{-6}$ \\
\hline Total Time of simulation $(\mathrm{s})$ & & $10^{-13}$ \\
\hline Initial Time Step (s) & \multicolumn{3}{|c}{} \\
\hline
\end{tabular}

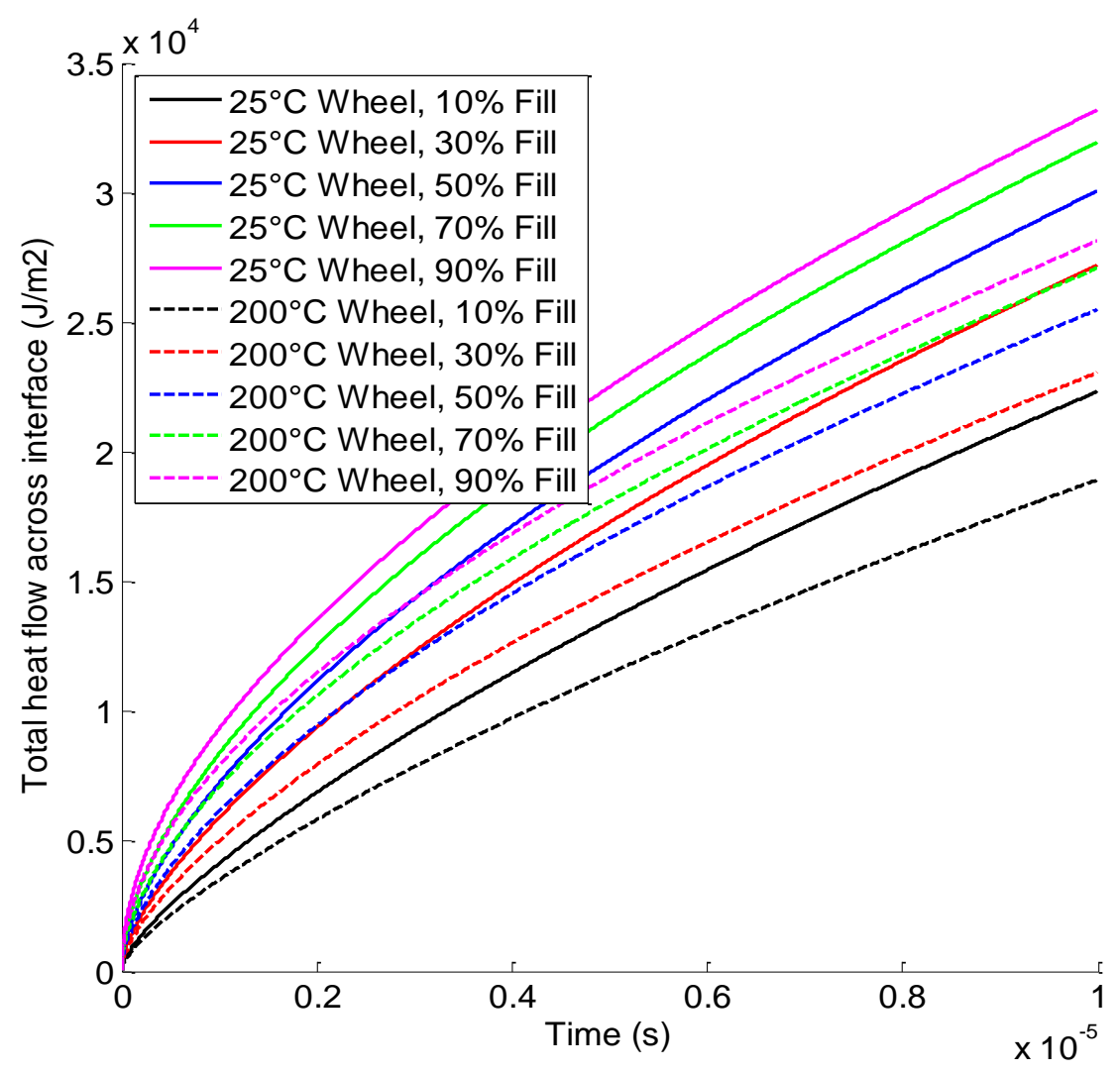

Figure 79 - Heat flow across interface with time. Wheel temperature and fill depth are varied. 
Interfacial heat transfer coefficients calculated from the heat fluxes obtained from the simulation range from $\sim 2 \times 10^{6}$ to $\sim 3 \times 10^{6} \mathrm{~W} / \mathrm{m}^{2} \mathrm{~K}$ when the simulation was run for $10 \mu \mathrm{s}$ (see Figure 80 ). It should be noted that no change in heat transfer coefficient was observed with changing wheel temperature. The equation used to calculate the heat transfer coefficient is shown below:

$$
H_{\text {int }}=\frac{q}{A * t * \Delta T}
$$

Where $\mathrm{H}_{\text {int }}$ is the interfacial heat transfer coefficient, $\mathrm{q}$ is the total heat flow across the interface, $A$ is the apparent area of the interface, $t$ is the time in which heat flow was measured, and $\Delta T$ is the difference between initial melt temperature and initial wheel temperature.

For comparison, Wang and Matthys [91] reported initial interfacial heat transfer coefficients (either $\mathrm{Ni}$ or $\mathrm{Cu}$ splats on various substrates) during splat cooling ranging from $10^{4}$ to $3 \times 10^{5} \mathrm{~W} / \mathrm{m}^{2} \mathrm{~K}$. This indicates that the model displays heat fluxes that are too high. This is not surprising, since the interface where the melt is directly in contact with the wheel was not assumed to have any interfacial resistance. If an interfacial resistance were added to the model, the observed heat transfer coefficient would approach that seen in experimental results. However, there is a lack of a good physical description of this phenomenon in the literature [126], implying that any factor would simply be an empirically determined coefficient. 


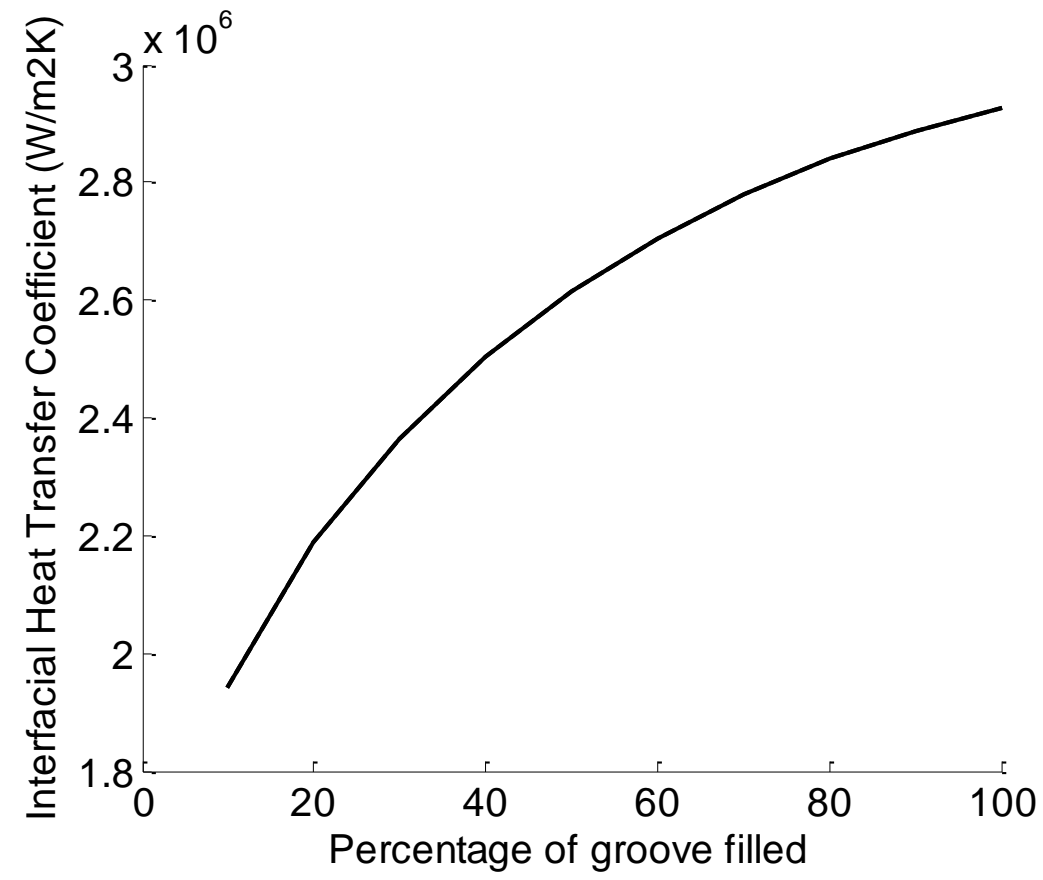

Figure 80 - Calculated interfacial heat transfer coefficient as a function of the percentage of the groove filled by the melt.

Temperature profiles using the parameters outlined in Table 2 and where the fill depth was maintained at $50 \%$ can be seen below in Figure 81 for various simulation times. 

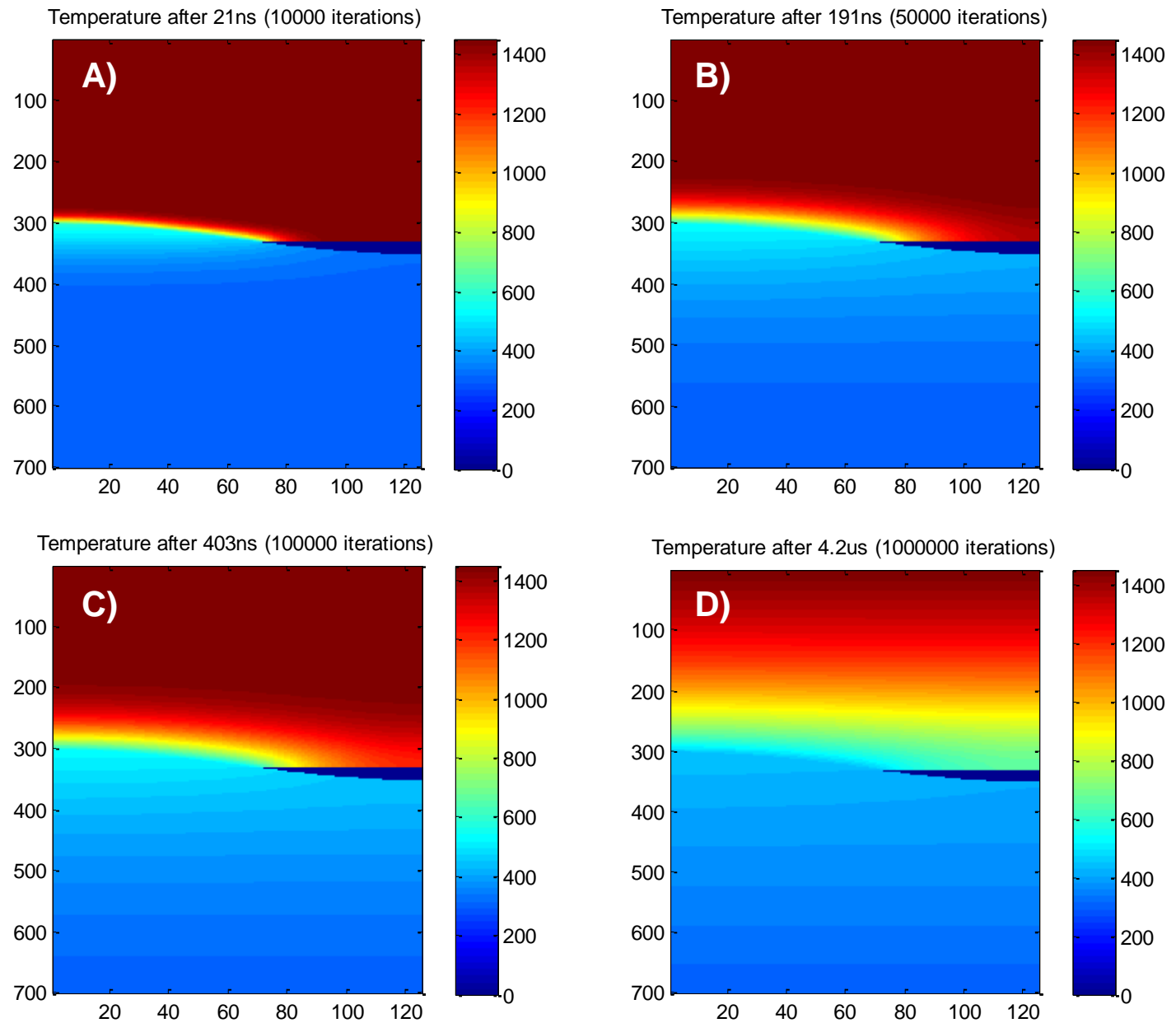

Figure 81 - Temperature (in K) profile of the matrix at a) 21 ns, b) 191 ns, c) 403 ns, and d) $4.2 \mu \mathrm{s}$.

Several improvements could be made on this model. One improvement would be utilizing temperature dependent material properties (e.g. heat capacity or thermal conductivity) when calculating temperature in order to improve the accuracy of the results (but this improvement would come at a significant computational time cost). The assumptions made concerning the surface condition of the wheel may not be entirely accurate either. The addition of an oxide layer between the wheel surface and melt would likely also improve the heat transfer coefficient accuracy. It was also experimentally shown in Chapter 5 that the uniformity of the surface conditions was 
not particularly constant, with regions where good wetting was seen, regions where poor wetting was seen, and regions with high concentrations of He bubbles. To more accurately model the overall behavior in the ribbon, different profiles could be used and the heat fluxes summed as a percentage of the type of region. The model, as it currently stands, also does not allow for crystallization of the melt. When this occurs, heat is released and would affect the temperature profile. By making the temperature calculation incorporate the effects of crystallization, the temperature profile would be more accurate. 


\section{Works Cited}

[1] J. F. Gieras, R.-J. Wang, and M. J. Kamper, Axial Flux Permanent Magnet Brushless Machines, 2nd Edition ed.: Springer, 2008.

[2] J. F. Gieras and M. Wing, Permanent Magnet Motor Technology: Design and Applications, 2nd Edition ed. New York: Marcel Dekker, 2002.

[3] D. Jiles, Introduction to Magnetism and Magnetic Materials, 2nd Edition ed. New York: Chapman and Hall/CRC, 1998.

[4] M. J. Kramer, Y. Xu, K. W. Dennis, I. E. Anderson, and R. W. McCallum, "Development of improved powder for bonded permanent magnets," IEEE TRANSACTIONS ON MAGNETICS, vol. 39, pp. 2971-2973, Sept. 2003.

[5] P. Campbell, "System Cost Analysis for an Interior Permanent Magnet Motor," Ames Laboratory IS-5191, 2008.

[6] C. Kittel, "Physical Theory of Ferromagnetic Domains," Reviews of Modern Physics, vol. 21, pp. 541-583, 1949.

[7] F. Bloch, "Zur Theorie des Austauschproblems und der Remanenzerscheinung der Ferromagnetika," Zeitschrift fur Physik A Hadrons and Nuclei, vol. 74, pp. 295-335, 1932.

[8] L. Neel, "Quelques proprietes des parois de domains elementaires ferromagnetiques," Cahiers de Physiques, vol. 25, pp. 1-20, 1944.

[9] J. D. Livingston, Driving force: the natural magic of magnets: Harvard University Press, 1996.

[10] C. Kittel, "Domain Theory and the Dependence of the Coercive Force of Fine Ferromagnetic Powders on Particle Size," Physical Review, vol. 73, pp. 810-811, 1948.

[11] J. J. Croat, J. F. Herbst, R. W. Lee, and F. E. Pinkerton, "High-energy product Nd-FeB permanent magnets," Applied Physics Letters, vol. 44, pp. 148-149, 1984.

[12] M. Sagawa, S. Fujimura, N. Togawa, H. Yamamoto, and Y. Matsuura, "New material for permanent magnets on a base of Nd and Fe (invited)." vol. 55: AIP, 1984, pp. 2083-2087.

[13] W. Rodewald, B. Wall, M. Katter, and K. Uestuener, "Top Nd-Fe-B magnets with greater than 56 MGOe energy density and 9.8 kOe coercivity," Magnetics, IEEE Transactions on, vol. 38, pp. 2955-2957, 2002.

[14] G. C. Hadjipanayis, R. C. Hazelton, and K. R. Lawless, "New iron-rare-earth based permanent magnet materials," Applied Physics Letters, vol. 43, pp. 797-799, 1983.

[15] F. Spada, C. Abache, and H. Oesterreicher, "Crystallographic and magnetic properties of rare earth-transition metal compounds based on boron," Journal of the Less Common Metals, vol. 99, pp. L21-L24, 1984.

[16] H. H. Stadelmaier, N. A. Elmasry, and S. Cheng, "Cobalt-free and samarium-free permanent magnet materials based on an iron-rare earth boride," Materials Letters, vol. 2, pp. 169-172, 1983.

[17] J. F. Herbst, J. J. Croat, and W. B. Yelon, "Structural and magnetic properties of Nd2Fe14B (invited)," Journal of Applied Physics, vol. 57, pp. 4086-4090, 1985.

[18] J. F. Herbst and W. B. Yelon, "Crystal and magnetic structure of Pr2Fe14B and Dy2Fe14B," Journal of Applied Physics, vol. 57, pp. 2343-2345, 1985. 
[19] J. F. Herbst, "R2Fe14B materials: Intrinsic properties and technological aspects," Reviews of Modern Physics, vol. 63, p. 819, 1991.

[20] G. Hilscher, R. Grössinger, S. Heisz, H. Sassik, and G. Wiesinger, "Magnetic and anisotropy studies of Nd-Fe-B based permanent magnets," Journal of Magnetism and Magnetic Materials, vol. 54-57, pp. 577-578, 1986.

[21] R. Grössinger, R. Krewenka, X. K. Sun, R. Eibler, H. R. Kirchmayr, and K. H. J. Buschow, "Magnetic phase transitions and magnetic anisotropy in $\mathrm{Nd} 2 \mathrm{Fe} 14-\mathrm{xCoxB}$ compounds," Journal of the Less Common Metals, vol. 124, pp. 165-172, 1986.

[22] J. D. Livingston, "Magnetic domains in sintered Fe-Nd-B magnets," Journal of Applied Physics, vol. 57, pp. 4137-4139, 1985.

[23] E. C. Stoner and E. P. Wohlfarth, "A Mechanism of Magnetic Hysteresis in Heterogeneous Alloys," Philosophical Transactions of the Royal Society of London. Series A, Mathematical and Physical Sciences, vol. 240, pp. 599-642, May 4, 1948 1948.

[24] J. Ormerod, "The physical metallurgy and processing of sintered rare earth permanent magnets," Journal of the Less Common Metals, vol. 111, pp. 49-69, 1985.

[25] M. Sagawa, S. Hirosawa, H. Yamamoto, S. Fujimura, and Y. Matsuura, "B-Fe-Nd Phase Diagram," in ASM Alloy Phase Diagram Center, P. Villars, Ed. Materials Park, $\mathrm{OH}$ : ASM International, 1987.

[26] B. Hallemans, P. Wollants, and J. R. Roos, "B-Fe-Nd Phase Diagram," in ASM Alloy Phase Diagram Center, P. Villars, Ed. Materials Park, OH: ASM International, 1995.

[27] R. B. Pond, "Metallic filaments and method of making same," United States: MARVALAND INC, 1958.

[28] T. R. Anantharaman and C. Suryanarayana, Rapidly solidified metals: a technological overview: Trans Tech Publications, 1987.

[29] W. J. Boettinger, S. R. Coriell, and R. F. Sekerka, "Mechanisms of microsegregationfree solidification," Materials Science and Engineering, vol. 65, pp. 27-36, 1984.

[30] J.-F. Ji and C. Chao, "A novel technique for manufacturing metal-bonded Nd-Fe-B magnets by squeeze casting," Metallurgical and Materials Transactions A, vol. 33, pp. 637-646, 2002.

[31] R. K. Mishra, "Microstructure of melt-spun Nd-Fe-B magnequench magnets," Journal of Magnetism and Magnetic Materials, vol. 54-57, pp. 450-456, 1986.

[32] T. Takeshita and K. Morimoto, "Anisotropic Nd-Fe-B bonded magnets made from HDDR powders," Journal of Applied Physics, vol. 79, p. 5, 1996.

[33] T. Takeshita, R. Nakayama, and T. Ogawa, "Rare Earth·Iron· Boron Magnet Powder and Process of Producing Same," U. S. P. Office, Ed., 1992, p. 57.

[34] D. Brown, B.-M. Ma, and Z. Chen, "Developments in the processing and properties of NdFeb-type permanent magnets," Journal of Magnetism and Magnetic Materials, vol. 248, pp. 432-440, 2002.

[35] S. Hirosawa, M. Uehara, S. Mino, N. Ishigaki, and T. Tomida, "New aspects of Nd-Fe--B-based hydrogenation-disproportionation-desorption-recombination powders and anisotropic bonded magnets made from them: Microstructure and magnetic properties (invited)," Journal of Applied Physics, vol. 81, pp. 4821-4826, 1997.

[36] Y. Kato, "Thermal stability of sintered and bonded rare-earth magnets," Journal of Applied Physics, vol. 85, pp. 4868-4870, 1999. 
[37] Y. Honkura, N. Hamada, and C. Mishima, "Process for Producing Anisotropic Magnet Powder." vol. US 2006/0048855 Al, U. S. P. Office, Ed., 2006.

[38] L. P. Barbosa, H. Takiishi, and R. N. Faria, "The effect of cobalt content on the microstructure of Pr-Fe-Co-B-Nb alloys and magnetic properties of HDDR magnets," Journal of Magnetism and Magnetic Materials, vol. 268, pp. 132-139, 2004.

[39] W. B. Kamb, "Theory of Preferred Crystal Orientation Developed by Crystallization under Stress," The Journal of Geology, vol. 67, pp. 153-170, 1959.

[40] W. B. Kamb, "The Thermodynamic Theory of Nonhydrostatically Stressed Solids," J. Geophys. Res., vol. 66, 1961.

[41] R. Lee, E. Brewer, and N. Schaffel, "Processing of Neodymium-Iron-Boron meltspun ribbons to fully dense magnets," Magnetics, IEEE Transactions on, vol. 21, pp. 1958-1963, 1985.

[42] R. W. Lee, "Hot-pressed neodymium-iron-boron magnets," Applied Physics Letters, vol. 46, pp. 790-791, 1985.

[43] L. Li and C. D. Graham, Jr., "The origin of crystallographic texture produced during hot deformation in rapidly-quenched NdFeB permanent magnets," Magnetics, IEEE

Transactions on, vol. 28, pp. 2130-2132, 1992.

[44] Y. Nozawa, K. Iwasaki, S. Tanigawa, M. Tokunaga, and H. Harada, "Nd-Fe-B dieupset and anisotropic bonded magnets (invited)," Journal of Applied Physics, vol. 64, pp. 5285-5289, 1988.

[45] M. Leonowicz and H. A. Davies, "Effect of Nd content on induced anisotropy in hot deformed Fe-Nd-B magnets," Materials Letters, vol. 19, pp. 275-279, 1994.

[46] Y. Liu, L. Xu, Q. Wang, W. Li, and X. Zhang, "Development of crystal texture in Nd-lean amorphous Nd9Fe85B6 under hot deformation." vol. 94: AIP, 2009, p. 172502.

[47] S. Rivoirard, D. Chateigner, P. De Rango, D. Fruchart, R. P. De La Bathie, and J. L. Soubeyroux, "Texture investigation of hot-forged Nd-Fe-B magnets," Philosophical Magazine A, vol. 80, pp. 1955 - 1966, 2000.

[48] W. Wu, H. Li, Y. Xie, and X. Zhang, "Effect of high pressure on microstructure of crystallizing amorphous Nd9Fe85B6 alloy," Journal of Rare Earths, vol. 26, pp. 741744, 2008.

[49] N. G. Akdogan, G. C. Hadjipanayis, and D. J. Sellmyer, "Anisotropic Sm-(Co,Fe) nanoparticles by surfactant-assisted ball milling," Journal of Applied Physics, vol. 105, pp. 07A710-3, 2009.

[50] N. G. Akdogan, W. Li, and G. C. Hadjipanayis, "Anisotropic Nd2Fe4B nanoparticles and nanoflakes by surfactant-assisted ball milling," Journal of Applied Physics, vol. 109, pp. 07A759-3.

[51] A. M. Gabay, N. G. Akdogan, M. Marinescu, J. F. Liu, and G. C. Hadjipanayis, "Rare earth-cobalt hard magnetic nanoparticles and nanoflakes by high-energy milling," Journal of Physics: Condensed Matter, vol. 22, p. 164213.

[52] A. I. Gusev and A. S. Kurlov, "Production of nanocrystalline powders by high-energy ball milling: model and experiment," Nanotechnology, vol. 19, p. 265302, 2008.

[53] J. P. L. Yiping Wang and Yang Li and Chuanbing Rong and, "Sm-Co hard magnetic nanoparticles prepared by surfactant-assisted ball milling," Nanotechnology, vol. 18, p. 465701, 2007. 
[54] D. Basset, P. Matteazzi, and F. Miani, "Designing a high energy ball-mill for synthesis of nanophase materials in large quantities," Materials Science and Engineering: A, vol. 168, pp. 149-152, 1993.

[55] K. Ohmori, L. Li, and C. D. Graham, Jr., "Effect of added Cu on the Nd-rich phase in hot-deformed NdFeB magnets," Magnetics, IEEE Transactions on, vol. 28, pp. 21392141, 1992.

[56] J. F. Herbst, C. D. Fuerst, R. K. Mishra, C. B. Murphy, and D. J. V. Wingerden, "Coercivity enhancement of melt-spun Nd-Fe-B ribbons using low-level $\mathrm{Cu}$ additions," 1991, pp. 5823-5825.

[57] D. J. Branagan, "A metallurgical approach toward alloying in rare-earth permanent magnet systems." vol. Doctor of Philosophy Ames, IA: Iowa State University, 1995.

[58] D. J. Branagan and R. W. McCallum, "Changes in glass formation and glass forming ability of $\mathrm{Nd} 2 \mathrm{Fe} 14 \mathrm{~B}$ by the addition of TiC," Journal of Alloys and Compounds, vol. 244, pp. 40-47, 1996.

[59] M. J. Kramer, L. H. Lewis, L. M. Fabietti, Y. Tang, W. Miller, K. W. Dennis, and R. W. McCallum, "Solidification, microstructural refinement and magnetism in Nd2Fe14B," Journal of Magnetism and Magnetic Materials, vol. 241, pp. 144-155, 2002.

[60] M. J. Kramer, C. P. Li, K. W. Dennis, R. W. McCallum, C. H. Sellers, D. J. Branagan, and J. E. Shield, "A generalized solidification model and microstructural verification for the Nd-Fe-B-Ti-C system processed by rapid solidification," 1997, pp. 4459-4461.

[61] O. Filip, R. Hermann, and L. Schultz, "Growth kinetics and TiC precipitation phenomena in Nd-Fe-B-Ti-C melts in dependence on cooling parameters and composition," Materials Science and Engineering: A, vol. 375-377, pp. 1044-1047, 2004.

[62] E. F. Kneller and R. Hawig, "The exchange-spring magnet: a new material principle for permanent magnets," IEEE Transactions on Magnetics, vol. 27, pp. 3588-3560, 1991.

[63] R. Coehoorn, D. B. de Mooij, and C. de Waard, "Meltspun permanent magnet materials containing Fe3B as the main phase," Journal of Magnetism and Magnetic Materials, vol. 80, pp. 101-104, 1989.

[64] L. Withanawasam, G. C. Hadjipanayis, and R. F. Krause, "Enhanced remanence in isotropic Fe-rich melt-spun Nd-Fe-B ribbons," Journal of Applied Physics, vol. 75, pp. 6646-6648, 1994.

[65] H. Ono, N. Waki, M. Shimada, T. Sugiyama, A. Fujiki, P. Yamamoto, and M. Tani, "Isotropic bulk exchange-spring magnets with $34 \mathrm{~kJ} / \mathrm{m} 3$ prepared by spark plasma sintering method," Magnetics, IEEE Transactions on, vol. 37, pp. 2552-2554, 2001.

[66] A. M. Gabay, Y. Zhang, and G. C. Hadjipanayis, "Die-upset hybrid Pr--Fe--B nanocomposite magnets," Applied Physics Letters, vol. 85, pp. 446-448, 2004.

[67] Y. Zhang, A. M. Gabay, and G. C. Hadjipanayis, "Microstructure of nanocomposite $\mathrm{R}-\mathrm{Fe}-\mathrm{B}$ die-upset magnets $(\mathrm{R}=\mathrm{Pr}, \mathrm{Nd})$ produced from mechanically milled powders," in Magnetics Conference, 2005. INTERMAG Asia 2005. Digests of the IEEE International, 2005, pp. 1653-1654. 
[68] H. Fukunaga, H. Nakayama, T. Kamikawatoko, T. Yanai, M. Nakano, F. Yamashita, S. Ohta, M. Itakura, and M. Nishida, "Magnetic properties of Nd-Fe-B/a-Fe multilayered thick film magnets," Journal of Physics: Conference Series, vol. 266, p. 012027.

[69] S. Yamamuro, M. Okano, T. Tanaka, K. Sumiyama, N. Nozawa, T. Nishiuchi, S. Hirosawa, and T. Ohkubo, "Direct Iron Coating onto Nd-Fe-B Powder by Thermal Decomposition of Iron Pentacarbonyl," Journal of Physics: Conference Series, vol. 266, p. 012050.

[70] Y. Wang and E. Wang, "Research on preparing compact bulk nanocomposite $\mathrm{Nd} 2 \mathrm{Fe} 14 \mathrm{~B} / \mathrm{a}-\mathrm{Fe}$ magnetic materials by hot extrusion," Journal of Magnetism and Magnetic Materials, vol. 312, pp. 220-223, 2007.

[71] W. Kurz and D. J. Fisher, Fundamentals of solidification: Trans Tech Publications, 1986.

[72] W. J. Boettinger and S. R. Coriell, "Microstructure Formation in Rapidly Solidified Materials," in Science and Technology of the Undercooled Melt. vol. 114, P. R. Sahm, H. Jones, and C. M. Adam, Eds. Dordrecht: Martinus Nijhoff Publishers, 1986, pp. 81-109.

[73] W. J. Boettinger and J. H. Perepezko, "Fundamentals of Solidification at High Rates," in Rapidly solidified alloys: processes, structures, properties, applications, H. H. Liebermann, Ed.: M. Dekker, 1993, pp. 17-78.

[74] M. J. Aziz, "Model for solute redistribution during rapid solidification," Journal of Applied Physics, vol. 53, pp. 1158-1168, 1982.

[75] C. Kuhrt, L. Schultz, K. Schnitzke, S. Hock, and R. Behrensmeier, "High-temperature compressive plastic deformation of Nd2Fe14B single crystals," Applied Physics Letters, vol. 59, pp. 1418-1420, 1991.

[76] D. Dadon, Y. Gefen, and M. Dariel, "The texture of melt spun Fe76Nd16B8 ribbons," Magnetics, IEEE Transactions on, vol. 23, pp. 3605-3606, 1987.

[77] J. F. Herbst, J. J. Croat, and W. B. Yelon, "Structural and magnetic properties of Nd2Fe14B (invited)." vol. 57: AIP, 1985, pp. 4086-4090.

[78] V. I. Tkatch, A. M. Grishin, and V. V. Maksimov, "Estimation of the heat transfer coefficient in melt spinning process," Journal of Physics: Conference Series, vol. 144, p. 012104, 2009.

[79] S. P. H. Marashi, A. Abedi, S. Kaviani, S. H. Aboutalebi, M. Rainforth, and H. A. Davies, "Effect of melt-spinning roll speed on the nanostructure and magnetic properties of stoichiometric and near stoichiometric Nd-Fe-B alloy ribbons," 2009, p. 115410.

[80] M. J. Kramer, Y. Tang, K. W. Dennis, and R. W. McCallum, "Effects of Quenching Environment on the Structure of Melt- Spun Nd2Fe14B," MRS Online Proceedings Library, vol. 577, pp. null-null, 1999.

[81] L. H. Lewis, S. M. Collins, M. J. Kramer, and C. C. H. Lo, "Solidification, quenching gas and magnetic properties in melt-spun Nd2Fe14B," Magnetics, IEEE Transactions on, vol. 37, pp. 2486-2488, 2001.

[82] E. Ferrara, A. Stantero, P. Tiberto, M. Baricco, D. Janickovic, L. Kubicar, and P. Duhaj, "Magnetic properties and surface roughness of Fe64Co21B15 amorphous 
ribbons quenched from different melt temperatures," Materials Science and Engineering: A, vol. 226â€"228, pp. 326-330, 1997.

[83] V. I. Tkatch, A. I. Limanovskii, S. N. Denisenko, and S. G. Rassolov, "The effect of the melt-spinning processing parameters on the rate of cooling," Materials Science and Engineering: A, vol. 323, pp. 91-96, 2002.

[84] G. X. Wang and E. F. Matthys, "Modelling of rapid solidification by melt spinning: effect of heat transfer in the cooling substrate," Materials Science and Engineering: A, vol. 136, pp. 85-97, 1991.

[85] W. S. Rasband, "ImageJ," Bethesda, Maryland, USA: U.S. National Institute of Health, 1997-2011.

[86] B. Karpe, B. Kosec, and M. Bizjak, "Modeling of heat transfer in the cooling wheel in the melt-spinning process," Journal of Achievements in Materials and Manufacturing Engineering, vol. 46, pp. 88-94, 2011.

[87] B. Karpe, B. Kosec, T. Kolenko, and M. Bizjak, "Heat transfer analyses of continuous casting by free jet meltspinning device," Metalurgija, vol. 50, pp. 13-16, 2011.

[88] D. J. Branagan and R. W. McCallum, "Altering the cooling rate dependence of phase formation during rapid solidification in the Nd2Fe14B system," Journal of Magnetism and Magnetic Materials, vol. 146, pp. 89-102, 1995.

[89] R. W. Powell, C. Y. Ho, and P. E. Liley, Thermal conductivity of selected materials. Washington: U.S. Dept. of Commerce, National Bureau of Standards; for sale by the Superintendent of Documents, U.S. Govt. Print. Off., 1966.

[90] R. Hanitsch, "Rare-earth iron permanent magnets," J. M. D. Coey, Ed.: Oxford University Press, 1996, p. 457.

[91] G. X. Wang and E. F. Matthys, "Experimental determination of the interfacial heat transfer during cooling and solidification of molten metal droplets impacting on a metallic substrate: effect of roughness and superheat," International Journal of Heat and Mass Transfer, vol. 45, pp. 4967-4981, 2002.

[92] S. J. Palmer, "The effect of temperature on surface tension," Physics Education, vol. 11, p. $119,1976$.

[93] M. Tomut and H. Chiriac, "Viscosity and surface tension of liquid Fe-metalloid glassforming alloys," Materials Science and Engineering: A, vol. 304â€“306, pp. 272-276, 2001.

[94] J. Lawrence, "Wetting and Bonding Characteristics of Selected Liquid Metals with a High-Power Diode-Laser-Treated Alumina Bioceramic," Proceedings: Mathematical, Physical and Engineering Sciences, vol. 460, pp. 1723-1735, 06/08 2004.

[95] M. Ramirez, L. Henneken, and S. Virtanen, "Oxidation kinetics of thin copper films and wetting behaviour of copper and Organic Solderability Preservatives (OSP) with lead-free solder," Applied Surface Science, vol. 257, pp. 6481-6488.

[96] D. L. Cocke, R. Schennach, M. A. Hossain, D. E. Mencer, H. McWhinney, J. R. Parga, M. Kesmez, J. A. G. Gomes, and M. Y. A. Mollah, "The low-temperature thermal oxidation of copper, $\mathrm{Cu} 3 \mathrm{O} 2$, and its influence on past and future studies," Vacuum, vol. 79, pp. 71-83, 2005.

[97] H. Wieder and A. W. Czanderna, "The Oxidation of Copper Films to CuO0.67," The Journal of Physical Chemistry, vol. 66, pp. 816-821, 1962. 
[98] R. W. McCallum, I. E. Anderson, Y. Xu, M. J. Kramer, K. Dennis, and W. Tang, "Permanent Magnet with Low or No Dysprosium for High Temperature Performance," U. S. P. Office, Ed., 2011.

[99] P. Tenaud, A. Chamberod, and F. Vanoni, "Texture in Nd-Fe-B magnets analysed on the basis of the determination of $\mathrm{Nd} 2 \mathrm{Fe} 14 \mathrm{~B}$ single crystals easy growth axis," Solid State Communications, vol. 63, pp. 303-305, 1987.

[100] L. Swartzendruber, "The Ag-Fe (Silver-Iron) system," Journal of Phase Equilibria, vol. 5, pp. 560-564, 1984.

[101] B. Toby, "EXPGUI, a graphical user interface for GSAS," Journal of Applied Crystallography, vol. 34, pp. 210-213, 2001.

[102] R. Ferro, A. Borsese, S. Delfino, R. Marazza, and A. Seminara, "Phase equilibria in the silver-neodymium system," Journal of the Less Common Metals, vol. 35, pp. 3944, 1974.

[103] O. D. McMasters, K. A. Gschneider, and R. F. Venteicher, "Crystallography of the silver-rich rare-earth-silver intermetallic compounds," Acta Crystallographica Section B, vol. 26, pp. 1224-1229, 1970.

[104] A. M. Mulokozi, I. Stapf, and E. Gebhardt, "The high temperature modifications of the compounds PrAg2 and NdAg2," Journal of the Less Common Metals, vol. 57, pp. P39-P45, 1978.

[105] O. Takeda, T. H. Okabe, and Y. Umetsu, "Phase equilibrium of the system Ag-Fe-Nd, and Nd extraction from magnet scraps using molten silver," Journal of Alloys and Compounds, vol. 379, pp. 305-313, 2004.

[106] F. Weitzer, A. Leithe-Jasper, P. Rogl, K. Hiebl, A. Rainbacher, G. Wiesinger, W. Steiner, J. Friedl, and F. E. Wagner, "Magnetism of ternary compounds RE6Fe13X; $\mathrm{RE}=\mathrm{Pr}, \mathrm{Nd} ; \mathrm{X}=\mathrm{Cu}, \mathrm{Ag}, \mathrm{Au}, \mathrm{Zn}, \mathrm{Cd}$, and $\mathrm{Hg}$," Journal of Applied Physics, vol. 75, pp. 7745-7751, 1994.

[107] W. Tang, K. W. Dennis, Y. Q. Wu, M. J. Kramer, I. E. Anderson, and R. W. McCallum, "Studies of new YDy-based R2Fe14B magnets for high temperature performance $(\mathrm{R}=\mathrm{Y}+\mathrm{Dy}+\mathrm{Nd})$," Magnetics, IEEE Transactions on, vol. 40, pp. 29072909, 2004.

[108] N. T. Oster, D. T. Cavanaugh, K. W. Dennis, M. J. Kramer, R. W. McCallum, and I. E. Anderson, "Effects of Ag additions on melt-spun RE2Fe14B microstructure and texture," in Magnetism and Magnetic Materials Scottsdale, AZ, 2011.

[109] A. C. Larson and R. B. V. Dreele, "General Structure Analysis System (GSAS)," in Los Alamos National Laboratory Report LAUR 86-748, 2000.

[110] H. Okamoto, "Ag-B (silver-boron)," Journal of Phase Equilibria, vol. 13, pp. 211212, 1992.

[111] L. H. Ungar and R. A. Brown, "Cellular interface morphologies in directional solidification. IV. The formation of deep cells," Physical Review B, vol. 31, pp. 59315940, 1985.

[112] D. C. Van Aken and H. L. Fraser, "The microstructures of rapidly solidified hypereutectic Al-Be alloys," Acta Metallurgica, vol. 33, pp. 963-974, 1985.

[113] S. Parker, R. Pollard, D. Lord, and P. Grundy, "Precipitation in NdFeB-type magnet materials," Magnetics, IEEE Transactions on, vol. 23, pp. 2103-2105, 1987. 
[114] S. F. H. Parker, P. J. Grundy, and J. Fidler, "Electron microscope study of precipitation in a niobium-containing (Nd, Dy)-Fe-B sintered magnet," Journal of Magnetism and Magnetic Materials, vol. 66, pp. 74-78, 1987.

[115] W. Rodewald and B. Wall, "Structure and magnetic properties of sintered Nd-Fe-NbB magnets," Journal of Magnetism and Magnetic Materials, vol. 80, pp. 57-60, 1989.

[116] P. Schrey, "On the precipitation of intermetallic phases in Nb-containing Nd-Fe-B magnets," Journal of Magnetism and Magnetic Materials, vol. 73, pp. 260-262, 1988.

[117] P. A. Withey, E. J. Devlin, J. S. Abell, and I. R. Harris, "Ageing effects in $\mathrm{Nd}(\mathrm{Dy}) \mathrm{Fe}(\mathrm{Nb}) \mathrm{B}$ alloys and magnets," Journal of Magnetism and Magnetic Materials, vol. 80, pp. 67-70, 1989.

[118] Z. Chen, Y. Q. Wu, M. J. Kramer, B. R. Smith, B.-M. Ma, and M.-Q. Huang, "A study on the role of $\mathrm{Nb}$ in melt-spun nanocrystalline Nd-Fe-B magnets," Journal of Magnetism and Magnetic Materials, vol. 268, pp. 105-113, 2004.

[119] Y. B. Kim, K. W. Lee, C. S. Kim, H. C. Shin, T. K. Kim, M. Okada, and M. Homma, "Nb-added high-coercivity Nd-Fe-B melt-spun ribbon with high remanence," Journal of Applied Physics, vol. 70, pp. 6477-6479, 1991.

[120] N. M. Fries, N. T. Oster, and I. E. Anderson, "Study of the Effect of Wheel Temperature on Microstructure of Nd2Fe14B Melt-Spun Ribbons," MS\&T Annual meeting Poster Session, 2011.

[121] R. Didden, J. Sigafoos, M. O'Reilly, and P. Sturmey, "A Multisite Cross-Cultural Replication of Unsuccessful Self-Treatment of Writer's Block," Journal of Applied Behavioural Analysis, vol. 40, pp. 773-773, 2007.

[122] D. Upper, "The unsuccessful self-treatment of a case of "writer's block"," Journal of applied behavior analysis, vol. 7, 1974.

[123] J. Larsen-Badse, "Influence of grit size on the groove formation during sliding abrasion," Wear, vol. 11, pp. 213-222, 1968.

[124] J. Larsen-Badse, "Some effects of specimen size on abrasive wear," Wear, vol. 19, pp. 27-35, 1972.

[125] M. Wadsak, M. Schreiner, T. Aastrup, and C. Leygraf, "A comparison of preparation methods of copper surfaces for in situ scanning force microscopy investigations," Applied Surface Science, vol. 157, pp. 39-46, 2000.

[126] G. Li and B. Thomas, "Transient thermal model of the continuous single-wheel thinstrip casting process," Metallurgical and Materials Transactions B, vol. 27, pp. 509$525,1996$. 